\title{
Complementary Metal Oxide Semiconductor Compatible Silicon-on-Insulator Optical Rib Waveguides with Local Oxidation of Silicon Isolation
}

\author{
By \\ Lynda Rowe, B.Eng. Carleton University \\ A thesis submitted to the Faculty of Graduate Studies and Research in partial fulfillment \\ of the requirements for the degree of Masters of Applied Science in Electrical \\ Engineering \\ Ottawa-Carleton Institute for Electrical and Computer Engineering \\ Department of Electronics \\ Faculty of Engineering \\ Carleton University
}

November 2006

C Copyright

2006, Lynda Rowe 


$\begin{array}{ll}\begin{array}{l}\text { Library and } \\ \text { Archives Canada }\end{array} & \begin{array}{l}\text { Bibliothèque et } \\ \text { Archives Canada }\end{array} \\ \begin{array}{l}\text { Published Heritage } \\ \text { Branch }\end{array} & \begin{array}{l}\text { Direction du } \\ \text { Patrimoine de l'édition }\end{array} \\ \begin{array}{l}\text { 395 Wellington Street } \\ \text { Ottawa ON K1A ON4 }\end{array} & \begin{array}{l}\text { 395, rue Wellington } \\ \text { Ottawa ON K1A ON4 } \\ \text { Canada }\end{array}\end{array}$

Your file Votre référence ISBN: 978-0-494-23345-0 Our file Notre référence ISBN: 978-0-494-23345-0

NOTICE:

The author has granted a nonexclusive license allowing Library and Archives Canada to reproduce, publish, archive, preserve, conserve, communicate to the public by telecommunication or on the Internet, loan, distribute and sell theses worldwide, for commercial or noncommercial purposes, in microform, paper, electronic and/or any other formats.

The author retains copyright ownership and moral rights in this thesis. Neither the thesis nor substantial extracts from it may be printed or otherwise reproduced without the author's permission.
AVIS:

L'auteur a accordé une licence non exclusive permettant à la Bibliothèque et Archives Canada de reproduire, publier, archiver, sauvegarder, conserver, transmettre au public par télécommunication ou par l'Internet, prêter, distribuer et vendre des thèses partout dans le monde, à des fins commerciales ou autres, sur support microforme, papier, électronique et/ou autres formats.

L'auteur conserve la propriété du droit d'auteur et des droits moraux qui protège cette thèse. $\mathrm{Ni}$ la thèse ni des extraits substantiels de celle-ci ne doivent être imprimés ou autrement reproduits sans son autorisation.
In compliance with the Canadian

Privacy Act some supporting forms may have been removed from this thesis.

While these forms may be included in the document page count, their removal does not represent any loss of content from the thesis.
Conformément à la loi canadienne sur la protection de la vie privée, quelques formulaires secondaires ont été enlevés de cette thèse.

Bien que ces formulaires aient inclus dans la pagination, il n'y aura aucun contenu manquant.

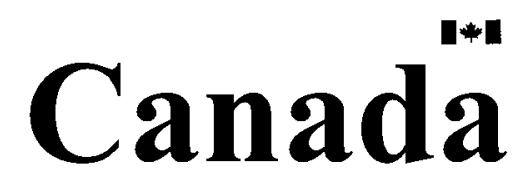




\begin{abstract}
The Local Oxidation of Silicon (LOCOS) technique is used for the first time to define optical rib waveguides in silicon-on-insulator (SOI) material. Optical mode simulation was used to determine rib geometries suitable for single-mode propagation in the 1550 $\mathrm{nm}$ optical telecommunications band. Test devices were then fabricated in SOI material with a Si film thickness near $3 \mu \mathrm{m}$. Cut-and-etchback optical testing of $3 \mu \mathrm{m}$ drawn width ribs showed the loss to be less than $1 \mathrm{~dB} / \mathrm{cm}$ at $1555 \mathrm{~nm}$. Unbalanced MachZehnder interferometers with Y-splitter junctions were also fabricated and tested with input wavelength swept from 1470 to $1580 \mathrm{~nm}$ and showed an extinction of 6-10 dB, demonstrating the ability of the LOCOS rib technique to produce more complex waveguide devices.
\end{abstract}




\section{Acknowledgments}

I would like to begin by thanking my thesis supervisor, Dr. Garry Tarr, for all his ongoing support and guidance throughout the completion of this project. Your insight into the world of silicon processing is very much appreciated!

I would also like to thank Carol Adams and Rob Vandusen for all of their help in preparing my samples in the Carleton University Microfabrication Facility. Many thanks to Edith Post and Winnie Ye at the National Research Council, and Michael Elsey and Dr. Andrew Knights at McMaster University for all of their assistance in testing my samples.

Finally, I would like to thank my friends and family for all of their support when I needed it the most. 


\section{Table of Contents}

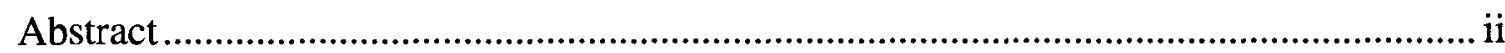

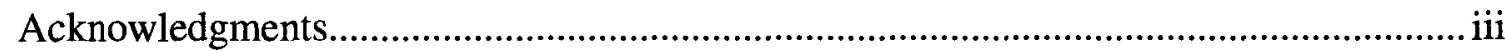

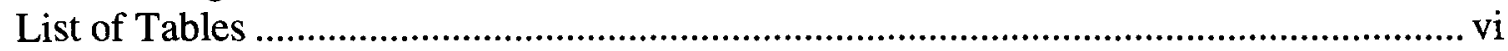

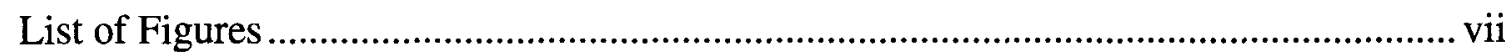

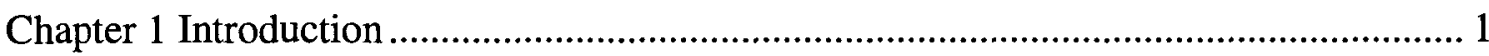

1.1 Silicon-on-Insulator as a Substrate for Optical Telecommunication and Optical

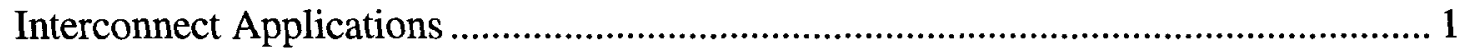

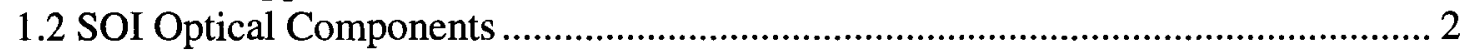

1.3 Integration of Optical and Electronic Components ........................................... 3

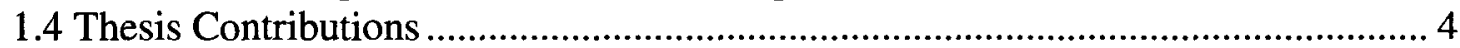

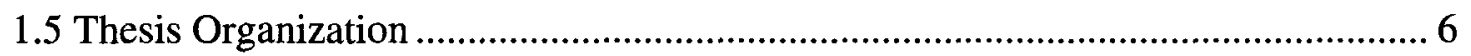

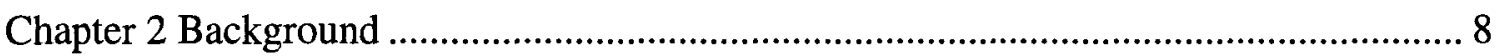

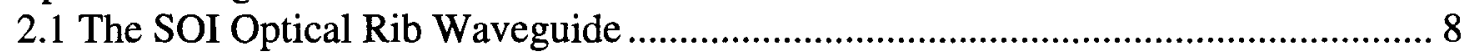

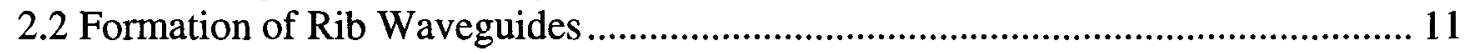

2.3 Local Oxidation of Silicon (LOCOS) Process and Features ................................. 13

2.4 LOCOS as a Rib Waveguide Fabrication Method............................................. 17

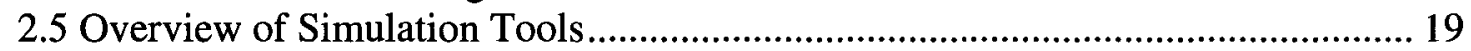

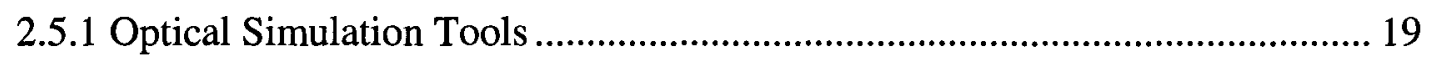

2.5.2 Process Flow Modeling - TSUPREM 4 …............................................... 21

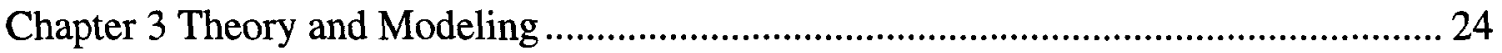

3.1 Confined Optical Modes in LOCOS waveguide ............................................. 24

3.1.1 Mode Solving With Apollo Photonic Solutions Suite …................................. 24

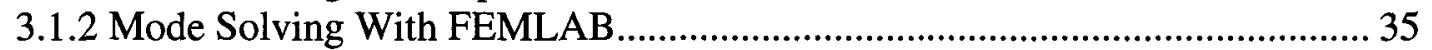

3.2 Waveguide Birefringence and Sidewall Angle .................................................. 42

3.3 Beam propagation in Unbalanced Mach-Zehnder Interferometer .......................... 45

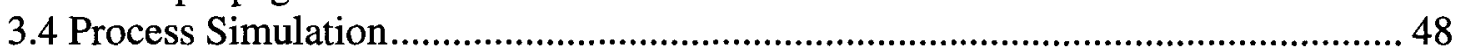

3.4.1 Conventional Local Oxidation of Silicon (LOCOS) Process ........................... 49

3.4.2 Polysilicon Buffered LOCOS (PBL) Process ................................................. 54

3.4.3 Sealed-Interface Local Oxidation (SILO) .................................................... 58

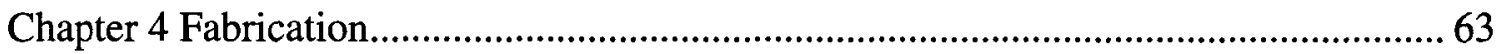

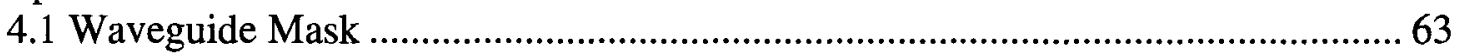

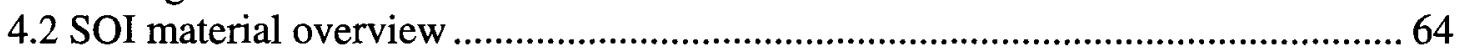

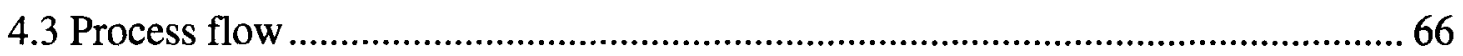

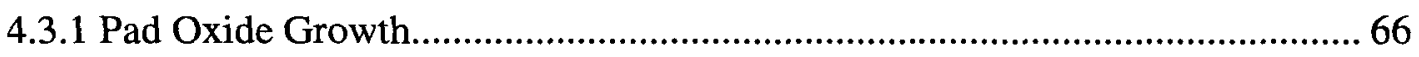

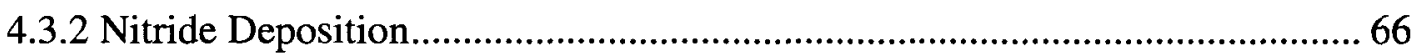

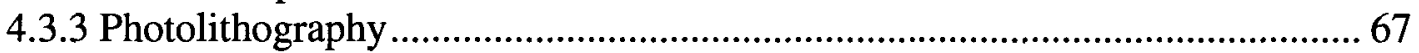

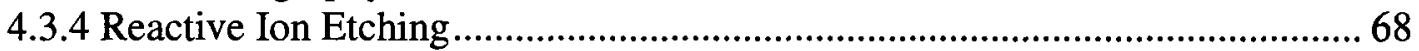

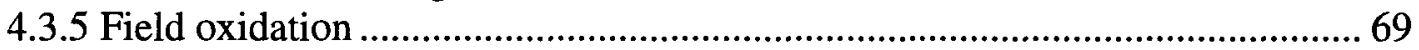

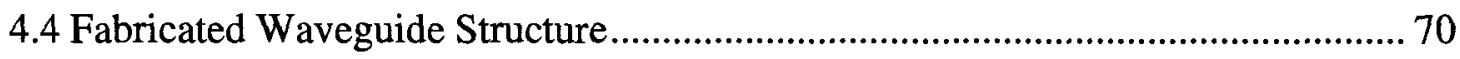


4.5 End Facet Preparation for Testing ………………........................................... 71

Chapter 5 Results and Discussion................................................................................... 74

5.1 Experiment Set-up and Waveguide Alignment Procedure ...................................... 74

5.2 Waveguide Mode Profiles (NRC and McMaster) ……………………………........ 77

5.3 Propagation Losses in $3 \mu \mathrm{m}$ and $4 \mu \mathrm{m}$ LOCOS waveguides (McMaster) .............. 79

5.4 Unbalanced Mach-Zehnder Interferometer Performance (NRC) ……................... 82

5.5 Balanced Y-splitter Performance (NRC) ............................................................... 84

Chapter 6 Conclusion and Recommendations ................................................................... 85

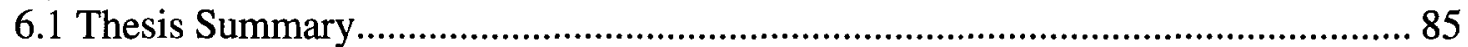

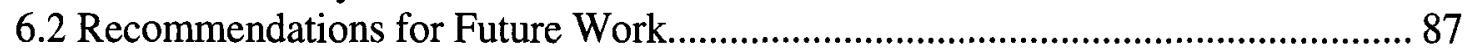

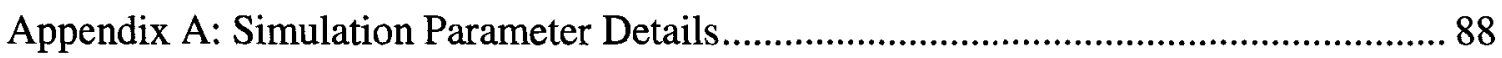

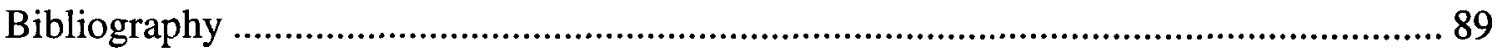




\section{List of Tables}

Table 3.1: Simulation parameter values for LOCOS waveguides in SEH (SOITEC) wafers. 25

Table 3.2: Refractive indices of LOCOS waveguide materials at $1550 \mathrm{~nm}$ wavelength. 25 Table 3.3: Simulation parameters used in FEMLAB for SEH (SOITEC) wafers . 36

Table 5.1: Output power level obtained with $1550 \mathrm{~nm}$ light of both polarizations launched into $3 \mu \mathrm{m}$ Y-splitter. 84

Table A.1: Simulation grid for Apollo simulations of LOCOS waveguide in SEH (SOITEC) substrates. 88 


\section{List of Figures}

Figure 2.1: SOI optical rib waveguide ......................................................................... 8

Figure 2.2: The basic LOCOS isolation structure widely used in CMOS integrated

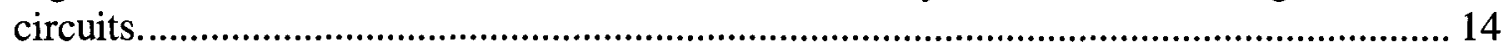

Figure 3.1: Cross-sectional view of waveguide structure used for optical simulation ..... 25 Figure 3.2: Fundamental TE waveguide mode with effective index of 3.46220 in a $2 \mu \mathrm{m}$ ridge in SEH material $(2.5 \mu \mathrm{m}$ silicon layer over a $1 \mu \mathrm{m}$ buried oxide layer) ……......... 27 Figure 3.3: Fundamental TM waveguide mode with effective index of 3.46453 in a $2 \mu \mathrm{m}$ ridge in SEH material $(2.5 \mu \mathrm{m}$ silicon layer over a $1 \mu \mathrm{m}$ buried oxide layer) …............. 28 Figure 3.4: Unconfined TE mode of the LOCOS waveguide in a $2 \mu \mathrm{m}$ ridge in SEH material $(2.5 \mu \mathrm{m}$ silicon layer over a $1 \mu \mathrm{m}$ buried oxide layer) .................................... 29 Figure 3.5: Unconfined TM mode of the LOCOS waveguide in a $2 \mu \mathrm{m}$ ridge in SEH material $(2.5 \mu \mathrm{m}$ silicon layer over a $1 \mu \mathrm{m}$ buried oxide layer) ....................................... 29 Figure 3.6: Second order TE mode of the LOCOS waveguide with an effective index of 3.45978 found at a ridge width of $4.1 \mu \mathrm{m}$ in SEH material........................................... 30 Figure 3.7: Second order TM mode of the LOCOS waveguide with an effective index of 3.46299 found at a ridge width of $5.9 \mu \mathrm{m}$ in SEH material............................................... 31 Figure 3.8: Fundamental TE waveguide mode with effective index of 3.47059 in a $2 \mu \mathrm{m}$ ridge in SOITEC material ( $3.4 \mu \mathrm{m}$ silicon layer over a $0.4 \mu \mathrm{m}$ buried oxide layer) ........ 32 Figure 3.9: Fundamental TM waveguide mode with effective index of 3.47154 in a $2 \mu \mathrm{m}$ ridge in SOITEC material ( $3.4 \mu \mathrm{m}$ silicon layer over a $0.4 \mu \mathrm{m}$ buried oxide layer) ........ 32 Figure 3.10: Unconfined TE mode of the LOCOS waveguide in a $2 \mu \mathrm{m}$ ridge in SOITEC material ( $3.4 \mu \mathrm{m}$ silicon layer over a $0.4 \mu \mathrm{m}$ buried oxide layer) .................................... 33 Figure 3.11: Unconfined TM mode of the LOCOS waveguide in a $2 \mu \mathrm{m}$ ridge in SOITEC material $(3.4 \mu \mathrm{m}$ silicon layer over a $0.4 \mu \mathrm{m}$ buried oxide layer) .................................... 33 Figure 3.12: Second order TE mode of the LOCOS waveguide with an effective index of 3.47021 found at a ridge width of $7.0 \mu \mathrm{m}$ in SOITEC material....................................... 34 Figure 3.13: Second order TM mode of the LOCOS waveguide with an effective index of 3.42907 found at a ridge width of $7.4 \mu \mathrm{m}$ in SOITEC material. 34 Figure 3.14: Cross-sectional view of LOCOS waveguide structure used for FEMLAB simulation. The only difference between this structure and the one in Figure 3.1 is the extension of the isolation oxide above the ridge to more accurately model the "bird's

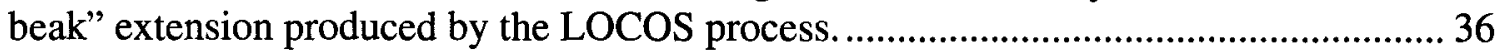
Figure 3.15: Fundamental TE mode in LOCOS waveguide with $2 \mu \mathrm{m}$ ridge width in SEH material as determined by simulation with FEMLAB..................................................... 37 Figure 3.16: Fundamental TM mode in LOCOS waveguide with $2 \mu \mathrm{m}$ ridge width in SEH material as determined by simulation with FEMLAB. .............................................. 38 Figure 3.17: First unconfined TE mode in LOCOS waveguide with $2 \mu \mathrm{m}$ ridge width in SEH material as determined by simulation with FEMLAB. 38 Figure 3.18: Fundamental TE mode of LOCOS waveguide with $2 \mu \mathrm{m}$ ridge in SOITEC material as determined by simulation with FEMLAB. 
Figure 3.19: Fundamental TM mode of LOCOS waveguide with $2 \mu \mathrm{m}$ ridge in SOITEC material as determined by simulation with FEMLAB..................................................... 40 Figure 3.20: First unconfined TE mode of LOCOS waveguide with $2 \mu \mathrm{m}$ ridge in SOITEC material as determined by simulation with FEMLAB......................................... 40

Figure 3.21: First unconfined TM mode of LOCOS waveguide with $2 \mu \mathrm{m}$ ridge in SOITEC material as determined by simulation with FEMLAB....................................... 41 Figure 3.22: Birefringence as a function of rib width for LOCOS, PBL, and RIE trench waveguides in SEH substrate. 43

Figure 3.23: Birefringence as a function of rib width for LOCOS, PBL, and RIE trench waveguides in SOITEC substrate. 44

Figure 3.24: Unbalanced MZI device with a path length difference of $16.8577 \mu \mathrm{m}$ constructed with LOCOS waveguides with a $4 \mu$ m ridge width. ....................................... 46 Figure 3.25: Attenuation from constructive and destructive interference in the MZI device of Figure 3.24 with TE (X) and TM (Y) polarized light.

Figure 3.26: Waveguide device cross-section produced using conventional LOCOS process used for isolation in the nMOS process in use in the Carleton University Microfabrication Facility. 50

Figure 3.27: Compressive stress $\sigma_{x x}\left(\mathrm{dyn} / \mathrm{cm}^{2}\right)$ due to oxidation in LOCOS waveguide structure.

Figure 3.28: Compressive stress $\sigma_{y y}\left(\mathrm{dyn} / \mathrm{cm}^{2}\right)$ due to oxidation in LOCOS waveguide structure.

Figure 3.29: Tensile stress $\sigma_{\mathrm{xx}}\left(\mathrm{dyn} / \mathrm{cm}^{2}\right)$ due to oxidation in LOCOS waveguide structure. 52

Figure 3.30: Tensile stress $\sigma_{y y}\left(\mathrm{dyn} / \mathrm{cm}^{2}\right)$ due to oxidation in LOCOS waveguide

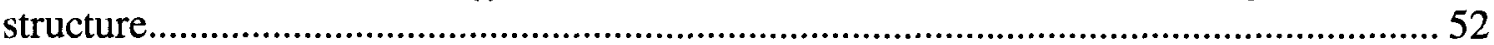

Figure 3.31: Waveguide device cross-section obtained with PBL process ........................55

Figure 3.32: Compressive stress $\sigma_{\mathrm{xx}}\left(\mathrm{dyn} / \mathrm{cm}^{2}\right)$ in PBL waveguide structure ................... 56

Figure 3.33: Compressive stress $\sigma_{\mathrm{yy}}\left(\mathrm{dyn} / \mathrm{cm}^{2}\right)$ in PBL waveguide structure ......................56

Figure 3.34: Tensile stress $\sigma_{\mathrm{xx}}\left(\mathrm{dyn} / \mathrm{cm}^{2}\right)$ in PBL waveguide structure ...........................5 57

Figure 3.35: Tensile stress $\sigma_{y y}\left(\mathrm{dyn} / \mathrm{cm}^{2}\right)$ in PBL waveguide structure ........................... 57

Figure 3.36: Waveguide device cross-section obtained with SILO process........................59

Figure 3.37: Compressive stress $\sigma_{\mathrm{xx}}\left(\mathrm{dyn} / \mathrm{cm}^{2}\right)$ in SILO waveguide structure................... 60

Figure 3.38: Compressive stress $\sigma_{\mathrm{yy}}\left(\mathrm{dyn} / \mathrm{cm}^{2}\right)$ in SILO waveguide structure...................60 60

Figure 3.39: Tensile stress $\sigma_{\mathrm{xx}}\left(\mathrm{dyn} / \mathrm{cm}^{2}\right)$ in SILO waveguide structure ............................ 61

Figure 3.40: Tensile stress $\sigma_{y y}\left(\mathrm{dyn} / \mathrm{cm}^{2}\right)$ in SILO waveguide structure........................... 61

Figure 4.1: Rib waveguide mask layout by Phil Waldron (McMaster University) ........... 64 Figure 4.2: SEM cross-section view of LOCOS waveguide structure after LOCOS stack removal at $15000 \mathrm{X}$ magnification. The white bars show a $1 \mu \mathrm{m}$ length......................... 70 Figure 4.3: Side view of tapered optical fibre butt-coupled to waveguide end facet ....... 71

Figure 5.1: Experimental setup used for optical testing at National Research Council

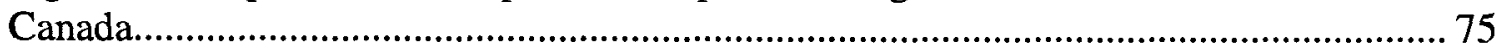

Figure 5.2: Fundamental TE mode observed for $3 \mu \mathrm{m}$ wide LOCOS isolated rib waveguide 
Figure 5.3: Fundamental TM mode observed in $3 \mu \mathrm{m}$ wide LOCOS isolated rib waveguide

Figure 5.4: Intensity distribution within guided mode in $3 \mu \mathrm{m}$ wide LOCOS isolated rib waveguide

Figure 5.5: Measured power $(\mathrm{dBm})$ vs. sample length $(\mathrm{cm})$ from cutback measurements

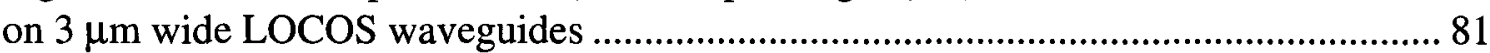
Figure 5.6 : Measured power $(\mathrm{dBm})$ vs. sample length $(\mathrm{cm})$ from cutback measurements

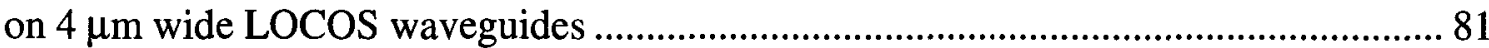
Figure 5.7: Power vs. wavelength for light of both polarizations launched into unbalanced MZI device. 


\section{Chapter 1 Introduction}

\subsection{Silicon-on-Insulator as a Substrate for Optical Telecommunication and Optical Interconnect Applications}

Since the 1970's the use of optical carriers for communications has become

commonplace as a means of transmitting large amounts of data over large distances

[Agra02]. With the introduction of the Internet in the 1990's, we have seen a shift in the use of optical communications from primarily voice-based communication to data-based communication [Cisco04]. This shift toward data-centric applications has resulted in a need for higher bandwidth, while the global nature of these applications has made longhaul transmission a key requirement. In order to meet these requirements, optical systems employ wavelength division multiplexing techniques with carriers in the $1532-1563 \mathrm{~nm}$ range as specified by ITU. Most recently, the increased demand for bandwidth has led to the deployment of fibre-to-the-home systems in which optical fibres are used to provide high bandwidth directly the end user. In addition to the typical long-haul telecommunications requirements, these systems must also be compact in size and available at a low cost in order to meet the needs of the consumer electronics market.

Another similar application of optical carriers to achieve rapid signal transmission is the optical interconnect. In this case, Moore's law has reduced the dimensions of the integrated circuit to a point where the delays seen by electrical signals as they pass through metal interconnect are becoming increasingly significant and limit the maximum operating frequency of digital circuits. By employing light [Car05] rather than electrical current as the information carrying medium, higher operating frequencies can be achieved. Although $850 \mathrm{~nm}$ was chosen for compatibility with gallium arsenide light 
Chapter 1 Introduction

sources [Car05], longer wavelengths in the $1550 \mathrm{~nm}$ range would serve the same purpose and at these wavelengths, silicon is effectively transparent, allowing SOI waveguides to be used.

With these applications in mind, some of the world's leading silicon IC manufacturers such as Intel [Sal04], IBM [Car05], and STMicroelectronics [Hau05] have been putting considerable research and development effort into integrated optical components on a silicon-on-insulator substrate. Silicon-on-insulator (SOI), as its name implies, consists of a thin silicon film on top of an insulating silicon dioxide layer on a thick silicon wafer substrate which provides mechanical support. Standard VLSI processing methods can be used to produce microelectronic circuitry in the silicon film. In addition, the high refractive index contrast between silicon and silicon dioxide films enables miniaturization of photonic elements compared to older silica waveguide technologies. It is the ability to integrate both optical and electronic devices on a single chip and the low manufacturing costs associated with the use of the silicon-on-insulator substrate that makes it an ideal choice for optical interconnect and mass market optical telecommunication applications.

\subsection{SOI Optical Components}

Many optical devices have been developed in the silicon-on-insulator substrate in recent years and most of these devices are based upon a single fundamental waveguide element. The most commonly used of these waveguide structures is the rib waveguide (discussed in further detail in Chapter 2) in which the silicon film is partially etched away to produce a core rib structure surrounded by thinner slab regions to provide horizontal confinement of light. The insulating silicon dioxide substrate layer below and the air or 
Chapter 1 Introduction

silicon dioxide cladding layers above provide vertical confinement of light due to their lower refractive indices.

Both active and passive optical elements such as variable optical attenuators (VOAs), electro-optic switches, splitters, arrayed waveguide gratings (AWG), taper couplers and integrated detectors make use of the optical rib waveguide as a part of their structure. Furthermore, the same optical rib waveguide can also be used to connect these elements to form more complex photonic circuits. Once these optical elements are integrated with CMOS electronics, an even broader range of applications are possible including the lowcost fibre-to-the-home transceiver units and on-chip optical interconnect.

\subsection{Integration of Optical and Electronic Components}

In order to print the features of optical and electronic components, a process known as photolithography is used. Modern photolithography has a limited depth of field which means that the surface on which the photoresist is applied must be very nearly planar for the mask patterns to be focussed clearly to get an accurate transfer of these patterns. As the device geometries continue to shrink, ensuring accurate pattern transfer by photolithography is becoming more challenging as the surface planarity requirements become tighter and tighter to be able to focus the increasingly small geometrical features.

The optical rib waveguide mentioned in the previous section would cause problems with the photolithographic process as the etched slab regions surrounding the ribs create a non-planar surface. This would be particularly problematic given that modern CMOS processes have features that are much smaller than a micron. Indeed, the $90 \mathrm{~nm}$ generation is currently in production, the $65 \mathrm{~nm}$ generation is forecast for 2007 , and the $45 \mathrm{~nm}$ generation is forecast for 2010 [ITRS05]. A clear solution to this problem would 
Chapter 1 Introduction

be to create the slab regions only in a region within a few microns of the rib such that a planar surface exists in the surrounding region for further CMOS processing steps. In this way, both optical and electrical elements can be integrated on the same chip to provide savings in terms of cost and device area for complex optoelectronic systems.

Another major problem with non-planar wafer surfaces affecting the reliability of electrical connections is the metal step coverage. This is the measure of how well a metal layer retains its nominal thickness as it covers change in topography or step. The greater the height of the step, the more difficult it becomes to avoid an unwanted decrease in the metal thickness on the side of the step. Eventually, when the metal becomes too thin breaks in the metal interconnect lines will form and open circuits will occur which decrease the manufacturing yield.

\subsection{Thesis Contributions}

In this thesis, the creation of a rib waveguide structure that maintains a planar silicon surface compatible with CMOS processing will be demonstrated. In particular, the local oxidation of silicon process commonly used for device isolation in older CMOS technologies will be employed as a means of creating a localized slab region for the rib waveguides while maintaining a refractive index profile suitable for the confinement of light to the waveguide rib.

Although, this method of isolation was abandoned by much of the semiconductor industry after the $0.5 \mu \mathrm{m}$ CMOS generation, it continues to be used in some CMOS technologies where longer gate lengths are required such as Dalsa Semiconductor's highvoltage processes [Dal04]. The ability to integrate these high voltage devices would be particularly useful for optical MEMS where these high voltages are required. 
Chapter 1 Introduction

The use of an existing isolation process for defining the optical ribs also simplifies the manufacturing process and reduces the number masks required. Given that mask costs are on the order of $\$ 1.5 \mathrm{M}$ per design, at around the $100 \mathrm{~nm}$ generation, and are forecasted to rise in the coming years as feature sizes shrink [ITRS05], this can have a significant impact on manufacturing costs of optoelectronic devices.

The use of LOCOS isolation to define optical waveguides in silicon was proposed by Pearson et al [Pear01] in the context of a SiGe guide with silicon cladding. This structure is discussed in more detail in Chapter 2. SiGe layers can be grown by low-temperature strained layer epitaxy on silicon substrates, and the high index of $\mathrm{SiGe}$ compared to the $\mathrm{Si}$ cladding allows guiding in the SiGe. The structure proposed by Pearson et al was never fabricated, and is impractical since the thermal budget required to implement LOCOS will cause complete strain relaxation in the SiGe through dislocation formation, rendering the material useless for electronic device fabrication.

LOCOS isolation was used by MIT Lincoln Laboratories [Gei03] in the context of creating a fixed delay element for wide-band antenna nulling applications. However, the structure created in this project was too wide in comparison to its height to be singlemode [Gei03]. Instead MIT Lincoln Labs suggested that it would turn to a combination of reactive ion etching and LOCOS [Gei03] to form smooth low-loss structures suitable for single-mode operation.

Another similar structure is the trench isolated SOI optical rib waveguide [Tur04] in which trenches resembling those used for electrical isolation in the modern shallow trench isolated [Plu00] CMOS processes are etched to define a localized slab region and maintain the refractive index profile required for guiding. However, the surface 
Chapter 1 Introduction

roughness produced by the reactive ion etch process used in fabricating this structure is responsible for a significant scattering loss, whereas the smoother surface produced by oxidation [Lai99], [Lee01] would be expected to reduce this loss.

\subsection{Thesis Organization}

An overview of the reasons behind choosing silicon-on-insulator as a substrate for optical interconnect and optical telecommunications applications, the importance of the rib waveguide in SOI optical devices and the challenges of integrating the optical rib waveguide with electronics have been presented in this chapter.

Chapter 2 will present the background material required to understand the design of the CMOS-compatible optical rib waveguides in this project. In particular the SOI optical rib waveguide and the techniques used in its formation, the local oxidation of silicon (LOCOS) process used to obtain the planar CMOS-compatible silicon surface, the previous work done on similar waveguides formed by LOCOS, and an overview of the Apollo and FEMLAB optical simulators and of the TSUPREM4 process simulation tool will be presented.

Chapter 3 will present the optical mode simulations used to determine the confined optical modes in the LOCOS rib waveguide structure, the beam propagation simulations used to estimate the losses in the waveguides, and the process simulations used to determine the impact of various conditions encountered in the manufacturing process on the final structure obtained.

Chapter 4 will present the details of the fabrication process used to manufacture the waveguides such as the process flow and the details of the steps followed and a discussion of the resulting waveguide structure obtained. 
Chapter 1 Introduction

Chapter 5 will present the results of optical testing of the waveguide devices completed at McMaster University and at the National Research council. In particular, the guided mode profiles and losses obtained as well as the behaviour of more complex devices such as the Y-splitter and unbalanced Mach-Zehnder Interferometer (MZI) will be discussed.

Chapter 6 summarizes the results obtained and suggests areas for future work related to this topic. 


\section{Chapter 2 Background}

\subsection{The SOI Optical Rib Waveguide}

The main structure being studied in this project is the optical rib waveguide shown in Figure 2.1. As can be seen, this structure consists of 3 regions: a core silicon layer, a lower cladding of silicon dioxide, and an upper cladding of either silicon dioxide or air. Since the refractive index of silicon is much greater than that of the surrounding silicon dioxide or air layers, the light is confined to this silicon dioxide core layer based on the principle of total internal reflection.
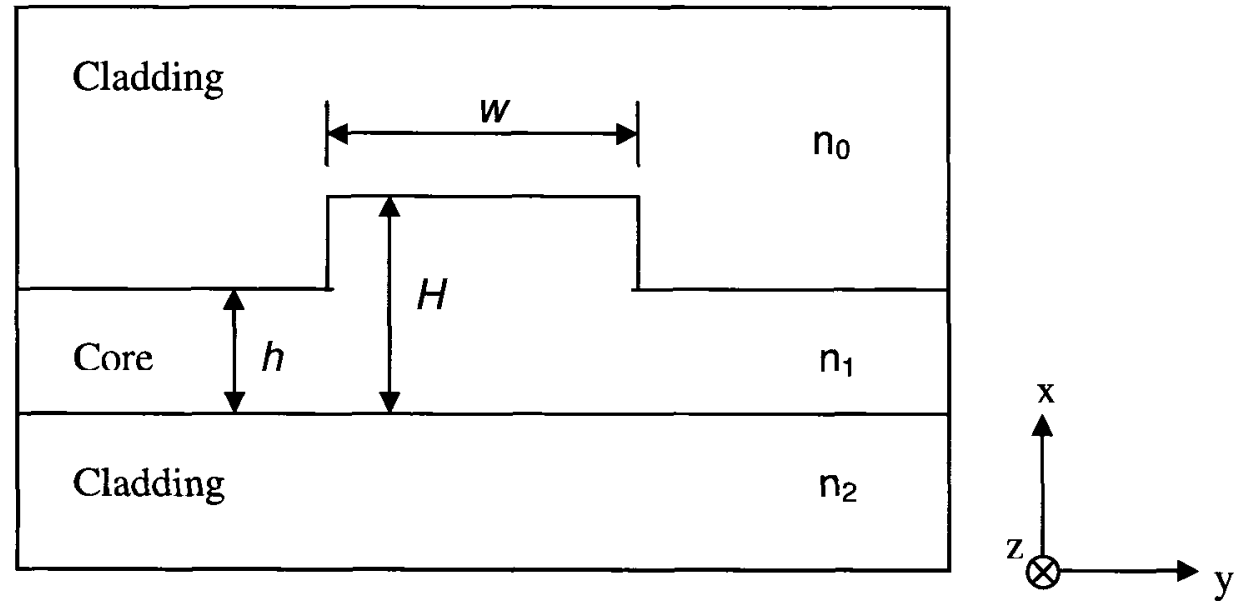

\section{Figure 2.1: SOI optical rib waveguide}

In addition, we can see that the silicon core layer is divided into a rib region of width $w$, and height $H$, and two surrounding silicon slab regions of height $h$. This variation in height is responsible for creating a slight variation in the effective refractive index seen by the light in the core to provide some additional confinement of the light in the 
Chapter 2 Background

horizontal direction. Since this effective index in the rib exceeds that in the surrounding silicon slab regions, the light is confined horizontally to the region under the rib.

To get a more detailed picture of how light propagates in optical waveguides, we must look to the Maxwell's equations for the electric and magnetic fields as light is an electromagnetic wave. For harmonic fields, these equations are:

$$
\begin{aligned}
& \nabla \times \mathbf{E}=i \omega \mu_{0} \mathbf{H} \\
& \nabla \times \mathbf{H}=-i \omega \varepsilon_{0} n^{2} \mathbf{E}
\end{aligned}
$$

where $\omega$ is the frequency of the wave, $\mu_{0}$ is the permeability of free space, $\varepsilon_{0}$ is the permittivity of free space, and $n$ is the refractive index. For a harmonic field, (2.1) and (2.2) simplify to the Helmholtz equations:

$$
\begin{aligned}
& \nabla^{2} \mathbf{E}+\omega^{2} n^{2} \mu_{0} \varepsilon_{0} \mathbf{E}=0 \\
& \nabla^{2} \mathbf{H}+\omega^{2} n^{2} \mu_{0} \varepsilon_{0} \mathbf{H}=0
\end{aligned}
$$

which can be solved either analytically or numerically using a partial differential equation solver (depending upon the complexity of the waveguide cross-section geometry) in the core, cover, and substrate regions of the waveguide to obtain the electric or magnetic field solutions which are known as the modes of the structure. These modes have two main forms, transverse electric (TE) and transverse magnetic (TM), labelled according to the orientation of the electric and magnetic field vectors in the structure. Transverse electric modes have their electric field vector oriented along the y-axis in Figure 2.1, parallel to the interface between materials in the waveguide, while their magnetic field vector is oriented along the $\mathrm{x}$-axis, normal to the interface between waveguide materials. Similarly, transverse magnetic modes have their electric field vector oriented along the $\mathrm{x}$ axis in Figure 1, normal to the interface between materials in the waveguide, and their magnetic field vector oriented along the $y$-axis, parallel to the interface between 
Chapter 2 Background

materials. In reality, however, the confined modes of the waveguide are not purely TE or TM polarized, but rather exist in the form of hybrid modes where electric and magnetic field components are present in both the $\mathrm{x}$ and $\mathrm{y}$ directions. However, in any mode, the magnitude of the both the electric field and the magnetic field components will be much larger along one direction than along the other such that we can still denote the mode as either approximately TE or approximately TM as dictated by the orientation of the dominant field components.

If an optical input pulse is able to excite more than one mode in the waveguide structure, the pulse will have broadened in the time domain once it reaches the other end of the waveguide due to the fact that each mode travels at a slightly different speed in the waveguide. Since this modal dispersion effect adversely affects the operation of any optical system using these waveguides, it must be reduced by employing a waveguide designed such that only a single optical mode can be excited. This single mode condition can be achieved through the careful choice of the waveguide width and slab height parameters, $w$ and $h$, according to the empirical equation:

$$
\frac{w}{H} \leq c+\frac{\frac{h}{H}}{\sqrt{1-\left(\frac{h}{H}\right)^{2}}}
$$

where $c$ is a constant. In the original work of Soref et al. [Sor91] the value of this constant, $c$, was 0.3 , although more recently it has been claimed that a value of -0.05 [Pog98] provides a better fit to experimental data. In addition, the slab height should meet the requirement:

$$
\frac{h}{H} \geq 0.5
$$

to avoid exciting multiple modes. 
Chapter 2 Background

Another major concern in the waveguide design is the loss of light intensity with the propagation distance. This can result from a number of physical mechanisms such as scattering, absorption, and radiation. A loss of much more than $1 \mathrm{~dB} / \mathrm{cm}$ is unacceptable as this would rapidly result in a very poor signal-to-noise ratio at a detector given that a typical optical circuit is a few centimetres in length [Reed04]. Since the absorption and scattering are mainly controlled through the choice of material, operating wavelength and the manufacturing process being used, the only component of the loss that can be controlled through careful design is radiation. Care must be taken to choose a cladding layer thickness that will prevent the loss of optical modes by radiation into the silicon substrate. Edge roughness on the ribs is responsible for scattering loss and must be carefully controlled during fabrication (see next section).

\subsection{Formation of Rib Waveguides}

For the traditional SOI rib waveguide structure of Figure 2.1, the most common way of defining the slab region is by reactive ion etching (RIE) [Plu00] of the upper silicon film. Photolithography is used to generate a protective coating of photoresist covering the rib region of the waveguide and then the reactive ion etching process is used to perform a dry anisotropic etch to produce the vertical sidewalls of the rib. Alternatively, a "hard mask" process could be used in which the rib region is protected by growing a thin layer of oxide and then patterning this oxide with photolithography and subsequent reactive ion etching. The oxide is then used as a mask for reactive ion etching of the underlying silicon to define the rib and slab regions. In either method, the protective photoresist or oxide layer is then removed to produce the final rib structure. 
Chapter 2 Background

One major disadvantage of these traditional SOI waveguides is the scattering loss obtained due to the roughness of the etched surface. In fact, due to the high refractive index contrast, $\Delta n$, between the core and substrate layers in the SOI rib waveguide structure, this problem becomes particularly significant as scattering loss is proportional to $\Delta n^{3}$ [Lee01]. This loss can be controlled through additional chemical etching or controlled oxidation steps to reduce the surface roughness [Lee01], [Lai99].

A similar waveguide structure could be produced by using a wet chemical etch in place of the RIE to produce the slab region [Lee01]. However, these wet chemical etching processes produce structures in which the sidewalls are sloped due to the difference in the etch rates in the vertical and horizontal directions. In addition, the chemicals employed may be incompatible with CMOS processing, particularly if they contain sodium or potassium, which are known to have adverse effects upon the threshold voltage in MOS transistors.

In both waveguide structures, if a silicon dioxide cladding layer is to be used, this layer is deposited by chemical vapour deposition after the rib has been etched and the protective layer of oxide or photoresist on the rib has been removed.

These traditional waveguide structures, however, are not suitable when electronic and optical components are to be integrated on the same chip due to the fact that they create a non-planar silicon surface that does not support the required additional photolithography steps needed to make electronic components. By employing another fabrication method such as local oxidation of silicon (LOCOS) to define a local slab region surrounding the rib, a planar silicon surface more suitable for the additional photolithography steps required when electronic devices are to be integrated on the same chip. In addition, since 
Chapter 2 Background

oxide has a lower refractive index than silicon, the total internal reflection required for guiding is preserved and the surface roughness problems associated with reactive ion etching can also be avoided. This modified waveguide structure and the LOCOS process will be described in greater detail in the subsequent sections.

Another method that provides a planar surface that supports photolithography is the trench isolated waveguide [Tur04]. This method involves etching only a narrow slab region of a few microns in width or trench similar to that used for shallow trench isolation in state-of-the-art submicron CMOS processes. In this way, the height of the silicon surface returns to the level of the rib so that the waveguide is separated from the remainder of the die by a trench, which can then be filled with oxide deposited by chemical vapour deposition and subsequently polished to obtain a completely flat surface over the entire die. This planar surface can then support including the small submicron MOSFET gate lengths of the state-of-the-art CMOS process on the same chip.

\subsection{Local Oxidation of Silicon (LOCOS) Process and Features}

Local Oxidation of Silicon (LOCOS) has been one of the most widely used methods for isolating active devices in CMOS integrated circuits since the 1970's [Wolf95]. This approach involves the selective growth of silicon dioxide in the field regions of the integrated circuit to raise the threshold voltage of the parasitic MOS devices formed when polysilicon or metal run over these field regions. This is done by covering the active device regions of the integrated circuit with a layer of silicon nitride which prevents or masks the oxidation of the silicon beneath it. Meanwhile, this silicon nitride layer is etched away over the field regions to allow the oxidation of the silicon in those areas to take place. 
Chapter 2 Background

The LOCOS process begins with the growth of a thin layer of thermal oxide known as pad oxide (typically $40-50 \mathrm{~nm}$ ) [Wolf90], which acts as a cushion to reduce thermal stresses between the silicon substrate and the silicon nitride masking layer due to the thermal expansion coefficient mismatch as well as the lifting of the nitride edges by the growing field oxide layer. Next, the thick silicon nitride masking layer is deposited and the pad oxide and nitride layers are etched away in the field regions. Finally, we are left with the stacked pad oxide and nitride masking layers covering only the active regions. The thick layer of thermal oxide in the field region is grown at high temperature (greater than the viscous flow threshold for silicon dioxide of $960{ }^{\circ} \mathrm{C}$ [Eer77]) to obtain the final isolation structure shown in Figure 2.2.

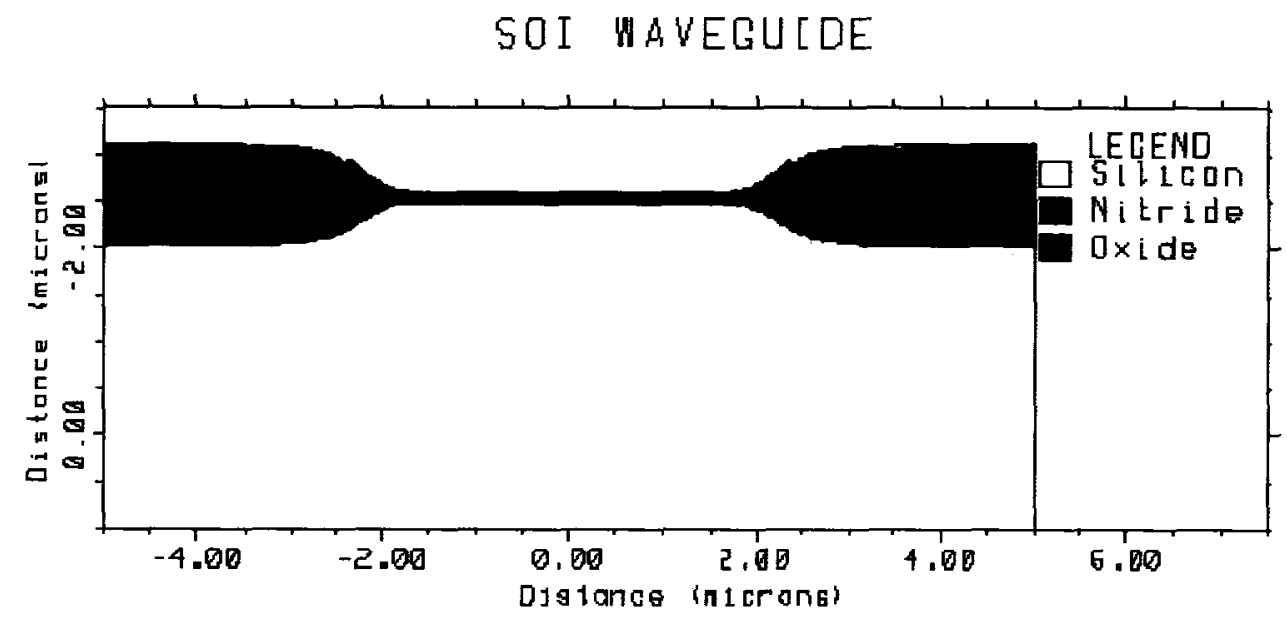

Figure 2.2: The basic LOCOS isolation structure widely used in CMOS integrated circuits.

Since the field oxide on this structure is grown without etching into the silicon, it is said to be partially recessed as some of the oxide lies above the level of the surface of the active region as can be seen in Figure 2.2. While this is not a perfectly planar surface, this 
Chapter 2 Background

LOCOS approach can support devices with geometries as small as $0.35 \mu \mathrm{m}$ [Plu00]. It is also important to note that the edges of the nitride mask region are lifted during the oxidation of the field region due to a lateral diffusion of oxygen under the nitride. This continued lateral diffusion occurs such that a wedge-shaped oxide region which is commonly referred to as a "bird's beak" that extends into the active device region is formed. This "bird's beak" encroachment also places a limit on the minimum size of the active regions that can be defined.

As a result of the limits placed on the active regions and thus the device geometry that can be formed using this traditional LOCOS process due to the formation of this "bird's beak" structure, throughout the 1980's and 1990's many research efforts focussed on modifications that reduce or eliminate the "bird's beak".

One very simple way of reducing the extent of this "bird's beak" region involves modifications to the thicknesses of the pad oxide and nitride layers. Generally, a reduction in the thickness of the pad oxide layer and an increase in the thickness of the nitride layer will result in a shorter "bird's beak", but care must be taken as this comes at the expense of increased thermal stress between the silicon nitride mask layer and the silicon substrate which causes dislocation damage in the silicon [Wolf90]. In order to avoid this dislocation damage when the field oxidation takes place at $1000{ }^{\circ} \mathrm{C}$, the pad oxide layer thickness must exceed $8 \mathrm{~nm}$ and the nitride layer thickness must not exceed three times the pad oxide thickness [Shi80].

One commonly used modification of the LOCOS process is the poly-buffered LOCOS (PBL) process [Wolf90] in which a thin layer of polysilicon is deposited on top of the pad oxide layer before a thicker nitride masking layer is deposited. In this process, the 
Chapter 2 Background

thickness of the pad oxide is typically reduced to only a few nanometres while the polysilicon layer is a few tens of nanometres thick. The thick nitride layer is fully etched while the polysilicon layer is only partially etched away (usually about halfway) to make the LOCOS stack before the field oxidation step is done. The polysilicon layer is able to squeeze out from under the nitride layer during the oxidation process to prevent the lifting of the nitride layer and oxidizes along with the silicon substrate to produce a "bird's beak" of substantially reduced length suitable for use with $0.7 \mu \mathrm{m}$ or smaller device applications [Wolf90].

Another modified form of LOCOS is the sealed-interface local oxidation method (SILO). It also reduces the length of the "bird's beak" by sealing the edges of the stack to prevent the lateral diffusion of oxygen responsible for the formation of the "bird's beak". In this case, a very thin layer of nitride of $10-20 \mathrm{~nm}$ in thickness is deposited directly on the silicon by CVD or grown by thermal nitridization of the silicon surface. Following that, a $25-30 \mathrm{~nm}$ thick oxide layer and a $150-200 \mathrm{~nm}$ thick nitride layer are deposited by CVD. Then this 3-layer stack is patterned by reactive ion etching (RIE) to expose the silicon over the field regions before they are oxidized [Wolf90]. The resulting structure is similar to the semi-recessed oxide obtained from the traditional LOCOS method except that a much steeper sidewall can be obtained due to the reduction in the bird's beak length to around $0.2 \mu \mathrm{m}$ per side [Wolf90].

A more complex modified LOCOS process is the sidewall-masked isolation (SWAMI) technique [Wolf90] used to produce a fully recessed field oxide. This process offers a reduced "bird's beak" lateral encroachment suited to more advanced submicron CMOS processes and a more planar surface as this is a fully recessed oxide as opposed to the 
Chapter 2 Background

semi-recessed oxides produced by other structures. This process involves forming a pad oxide and nitride stack in the same manner as for conventional LOCOS. The silicon in the field region is then etched to approximately one-half the desired field oxide thickness by using an orientation-dependent wet chemical etching process. This produces inclined sidewalls in the field region that help in reducing the stresses during field oxidation that contribute to dislocations. A second pad oxide layer is then grown and followed by the deposition of a second nitride layer and an oxide layer by CVD which provide a conformal coverage of the entire surface including the inclined sidewalls. These layers are subsequently plasma etched over the field region, leaving behind an oxide spacer which protects the nitride. The CVD oxide spacer is then removed by a wet chemical etch to reveal a silicon mesa covered with the oxide and nitride layers ready for the field oxidation.

As feature sizes dropped below $0.35 \mu \mathrm{m}$, the mainstream semiconductor industry turned to shallow trench isolation (STI) and abandoned the LOCOS process. However, LOCOS continues to be in use today in some older process technologies.

\subsection{LOCOS as a Rib Waveguide Fabrication Method}

As mentioned in section 2.1, a rib waveguide consists of three regions: a silicon core region surrounded by cladding layers above and below of lower refractive index for confinement of light by total internal reflection. In section 2.2 , it was pointed out that the conventional method of defining the rib structure by reactive ion etching was less than desirable due to the roughness of the sidewalls of the rib and due to the non-planar silicon surface created with this process. In the same section, it was suggested that a more attractive alternative would be to define the slab region by oxidation and only in a region 
Chapter 2 Background

localized to the rib as this would produce a more planar silicon surface and avoid the problems of surface roughness and its contribution to scattering loss.

An optical rib waveguide in which the rib was defined using the LOCOS process to create localized slab regions surrounding the rib that are covered by oxide was investigated by Pearson et al. [Pear01] for use on a silicon germanium waveguide layer surrounded by silicon cladding layers. This LOCOS process was selected due to the ease of integration with CMOS electronics, as it is often used to isolate active devices in CMOS processes, and due to the resemblance between an active device region surrounded by field oxide and the rib waveguide structure. This LOCOS process would be equally applicable to creating a rib waveguide in the silicon film of the SOI substrate material as it does not suffer from the chemical incompatibilities and thermal stress issues arising from the lengthy exposure to high temperatures [Sal04] that prevented this technique from being successfully applied to the silicon germanium waveguide.

\section{LOCOS was used to form silicon waveguides for wideband antenna nulling} applications by MIT Lincoln Laboratories [Gei03]. When using analog modulation of RF signals on an optical carrier, a fixed delay for signal processing in the optical domain could be achieved by creating a serpentine silicon waveguide structure using LOCOS. The reduced sidewall roughness obtained by oxidizing the silicon surface to form the silicon rib portion of the waveguide was used as a means of controlling the loss within the fixed delay lines to improve system performance. The structure obtained had a rib width of $1 \mu \mathrm{m}$ and a rib height of $1.5 \mu \mathrm{m}$ when an oxide was grown at $1100^{\circ} \mathrm{C}$ for 2 hours. Ultimately the waveguide structure proved too wide in comparison to the total 
Chapter 2 Background

height to be single-mode, so it was suggested that this approach be combined with silicon

RIE to obtain a structure with dimensions that are better suited to single-mode operation.

\subsection{Overview of Simulation Tools}

In this section, the simulation tools used for optical and process flow modeling will be described.

\subsubsection{Optical Simulation Tools}

In order to determine the optical characteristics of the waveguides that will be produced by the LOCOS process several simulations must be carried out. In particular, the optical modes of the waveguide structures must be simulated to determine the required dimensions of the waveguide (rib width and slab height) to obtain the desired single-mode propagation conditions. We have seen that the LOCOS structure has the "bird's beak" encroachment which creates angled sidewalls and rounded corners on the waveguide such that equation (2.5) relating rib width and slab height is not directly applicable. In addition, the loss in the waveguide structure as the light propagates along its length must also be found as this determines the maximum length of waveguide that can be used. In this case a beam propagation simulation is used to determine the loss of optical power as the light propagates along the length of the waveguide. An overview of the tools used in performing both the optical mode simulations and the beam propagation analyses will be presented in the remainder of this section.

Optical mode simulation was done with Apollo Photonic Solutions Suite [Apo02], which uses either the finite difference or effective index methods to solve for the propagation constant and field amplitude of a selected mode for a given polarization (TE, 
Chapter 2 Background

TM or both) in a waveguide cross-section whose layers and material properties are supplied by the user.

In the finite difference method, a rectangular grid is used throughout the simulation window and the Helmholtz equation is solved within the simulation window by computing numerical approximations to the differential and integral operations through the use of the five-point difference formula. The grid determines the step size in these numerical formulas as well as the accuracy of the overall result [Ste88].

With the effective index method [Chi86], [Chi96], the Helmholtz equation for the dominant field component in either the TE or TM polarization in the slab waveguide formed by the core and upper and lower cladding layers in both the rib and slab regions is solved. The resulting effective indices obtained are re-used as the refractive indices of core and cladding layers in solving the Helmholtz equation for the dominant field component in this new slab waveguide structure to determine the overall effective index and field profile.

FEMLAB [Com04], a finite element method [Kos85], [Kos92], [Mab81] partial differential equation solver was also used to solve the Helmholtz equation for the effective indices and field amplitudes of the TE and TM modes in the waveguide. The main purpose in using this solver was to provide additional confirmation of the existence of the optical modes in the waveguide found by the Apollo Photonic Solutions Suite.

In the finite element method [Kos85], [Kos92], [Mab81], a mesh consisting of triangles rather than squares or rectangles is used to partition the waveguide into a series of smaller regions for numerical computation of the solution to the Helmholtz equation. This discretization leads to the use of a matrix eigenvalue approach to writing the Helmholtz 
Chapter 2 Background

equation where the eigenvalues determine the effective indices of the electromagnetic modes in the structure and the eigensolutions are the field components. Gaussian elimination is the method used to solve the matrix equation [Com04].

The beam propagation method involves solving the Helmholtz equation in the direction of propagation in a waveguide device once the solution in the transverse direction is known. Since this is a first order differential equation in $\mathrm{z}$, the direction of propagation, the solution is clearly involves taking the integral in $\mathrm{z}$ [Van81]. As the form of this integral is very complicated [Van81], numerical approaches such as the finite difference algorithm are used to obtain its solution [Chu91]. This is most likely the approach used by Apollo Photonic Solutions Suite [Apo02] because, as with the finite difference method used to obtain the mode profile, a rectangular grid is used here ( $y-z$ plane $)$ and the effective indices of the slab and core regions of the waveguides are used in place of the refractive indices of the materials. The initial solution used would be the sinusoid in $y$ with an exponential decay in the slab regions, and the propagation in $\mathrm{z}$ is computed by solving the first order differential with the finite difference formulas for the required integral and differentials.

\subsubsection{Process Flow Modeling - TSUPREM 4}

In addition to the optical mode and beam propagation simulations needed to find the correct device dimensions, process flow modeling must also be carried out to find the impact of processing temperature, duration of processing steps, and LOCOS stack thicknesses on the final device structure obtained.

SUPREM was developed at Stanford University in an effort to reduce the need for costly and time-consuming empirical approaches to developing and optimizing VLSI 
Chapter 2 Background

technology through the understanding and implementation of accurate models of all of the physical processes involved in IC manufacturing in the form of a comprehensive computer program [Ant79], [Ho83]. This program can then be used to predict the form of any device structure on the basis of simulating the manufacturing steps required for its formation [Ho83]. Each generation of this computer program has drawn from the computer models based on the understanding of the manufacturing processes available at the time [Ho83] and now it has several commercial variants including Athena and TSUPREM.

TSUPREM-4 is the most recent commercial variant of this computer program which simulates the processing steps used in the manufacturing of silicon integrated circuit devices such as ion implantation, chemical pre-deposition through the surface, oxidation, dopant drive-in, epitaxial growth, etching, and material deposition. This tool models the addition and re-distribution of impurities within a 2-dimensional slice perpendicular to the surface of the silicon wafer. A typical simulation consists of specifying a sequence of processing steps with their associated times, temperatures, ambients, deposited materials, and implant doses and energies. The output of these simulations can include onedimensional dopant profiles, layer thicknesses and boundaries, mechanical stresses due to oxidation, thermal cycling, or film deposition, and electrical parameters [Syn05].

Since we are using the LOCOS process that is typically used in silicon integrated circuit manufacturing for providing the isolation between active devices for defining our waveguide ribs in the silicon film of the SOI wafer, we can easily simulate it with TSUPREM-4. By creating a script which includes all of the deposition, etching, diffusion and annealing stages and the temperature and duration of each one, the software can read 
Chapter 2 Background

this script to simulate each step and determine the boundaries between the layers in the LOCOS structure as well as the stresses in the structure due to the oxidation which can affect the birefringence of the waveguide structure. Plots of a 2-dimensional cross-section (i.e. Figure 2.2) through the final structure and the stresses within the structure due to the oxidation can be produced.

A detailed description of this simulation and the results obtained will be provided in Chapter 3. 


\section{Chapter 3 Theory and Modeling}

Several computer simulations with the tools described in section 2.5 were carried out to predict the behaviour of the LOCOS isolated waveguide before fabricating any prototype devices. In this section, the set-up of the simulations as well as the results obtained will be described. In a later chapter, these simulated results will be compared with the actual results obtained from the experimental prototype devices.

\subsection{Confined Optical Modes in LOCOS waveguide}

\subsubsection{Mode Solving With Apollo Photonic Solutions Suite}

In the previous sections, it has been mentioned that single mode waveguides would be most useful as this would avoid the problem of modal dispersion. We have seen that to find the modes that are confined within the optical rib waveguide structure, the Helmholtz equation must be solved in two-dimensions. Apollo Photonic Solutions Suite [Apo02] can solve this equation numerically.

To perform the simulation, a ridge waveguide structure with a sidewall angle of $60^{\circ}$ was defined in Apollo as shown in Figure 3.1. This angled sidewall is used to approximate the "bird's beak" extension produced by the lifting of the nitride mask and lateral diffusion of oxygen in the LOCOS process that can be seen later in the process simulation in section 3.4.1. In reality, this extension and the corners of the waveguide are more rounded and the partially recessed oxide will extend above the ridge with a similar rounded and tapered shape, although both of these effects will have little impact on the actual mode shape confined within the waveguide. 


\begin{tabular}{|l|l|l|}
\hline air & $\mathrm{DC}$ \\
\hline oxide & & \\
\hline channel & & \\
\hline buried oxide & $\mathrm{DS}$ \\
\hline substrate & \\
\hline
\end{tabular}

Figure 3.1: Cross-sectional view of waveguide structure used for optical simulation

\begin{tabular}{|l|l|l|}
\hline Name & Parameter & Value \\
\hline Channel Width & W & 2 to $6 \mu \mathrm{m}(2$ to $6 \mu \mathrm{m})$ \\
\hline Channel Height & HR & $2.5 \mu \mathrm{m}(3.4 \mu \mathrm{m})$ \\
\hline Etch Depth & H & $0.45 \mu \mathrm{m}(0.45 \mu \mathrm{m})$ \\
\hline Left Sidewall Angle & LA & $60^{\circ}\left(60^{\circ}\right)$ \\
\hline Right Sidewall Angle & RA & $60^{\circ}\left(60^{\circ}\right)$ \\
\hline Cover thickness & DC & $10 \mu \mathrm{m}(10 \mu \mathrm{m})$ \\
\hline Combined channel and field oxide thickness & D2 & $2.55 \mu \mathrm{m}(3.45 \mu \mathrm{m})$ \\
\hline Buried oxide thickness & D1 & $1 \mu \mathrm{m}(0.4 \mu \mathrm{m})$ \\
\hline Substrate thickness & DS & $10 \mu \mathrm{m}(10 \mu \mathrm{m})$ \\
\hline
\end{tabular}

Table 3.1: Simulation parameter values for LOCOS waveguides in SEH (SOITEC) wafers

\begin{tabular}{|l|l|}
\hline Material & Refractive Index \\
\hline air & 1.00 \\
\hline isolation oxide & 1.46 \\
\hline channel (silicon) & 3.48 \\
\hline buried oxide & 1.46 \\
\hline substrate (silicon) & 3.48 \\
\hline
\end{tabular}

Table 3.2: Refractive indices of LOCOS waveguide materials at $1550 \mathrm{~nm}$ wavelength. 
The effective index method was used to find an initial estimate of the propagation constant and optical intensity which were then used as a starting point for the finite difference method of solving the Helmholtz equation to further refine these results. Ridge widths ranging from 2 to $4 \mu \mathrm{m}$ were simulated in SOI wafers with a $2.5 \mu \mathrm{m}$ silicon film over a $1 \mu \mathrm{m}$ buried oxide layer and a $3.4 \mu \mathrm{m}$ silicon film over a $0.4 \mu \mathrm{m}$ buried oxide layer. On each of these SOI substrates field oxides of $1 \mu \mathrm{m}$ in thickness were assumed, which corresponds to an etch depth of roughly $0.45 \mu \mathrm{m}$ for the slab region as the silicon thickness consumed in oxidation is equal to approximately $45 \%$ of the total oxide thickness. These simulation parameters are summarized in Table 3.1 and the refractive indices of the materials are shown in Table 3.2.

Another important consideration is the conditions at the boundaries of the simulation window as these will affect the field amplitudes obtained. Ideally, these boundaries should allow the fields to pass through as though the boundary does not exist, since, in reality, no such boundaries exist to restrict the propagation of the fields. In the waveguide simulations, perfectly matched layer boundaries are used around the edges of the simulation window as these layers will allow the fields to pass through and prevent reflections such that a realistic field profile within the waveguide can be obtained. In these simulations, eight of these perfectly matched layers were used on the upper and lower edges of the simulation window. Forty perfectly matched layers were used on the left and right edges of the simulation window.

Finally, the choice of grid and window size for the simulation will also affect the quality of the result obtained. For this simulation, 150 evenly spaced grid points were 
Chapter 3 Theory

used over the width of the simulation window in the horizontal direction. In the vertical direction, the grid points were not distributed evenly with respect to the height of the simulation window. Instead these points were spaced evenly within each of the layers. More grid points were used within the silicon core region than in the air, silicon dioxide cladding and silicon substrate layers to obtain a more accurate mode profile region. In particular, the grid size will influence the stability of the finite difference algorithm used to refine the estimated field and propagation constants. The actual grid parameters used are shown in Table A.1 of Appendix A.

Finally, the TE and TM fundamental mode profiles were found at a wavelength of 1.55 $\mu \mathrm{m}$ for a $2 \mu \mathrm{m}$ ridge waveguide in SEH material as shown in Figure 3.2 and Figure 3.3 with effective indices of refraction of 3.46220 and 3.46453 respectively.

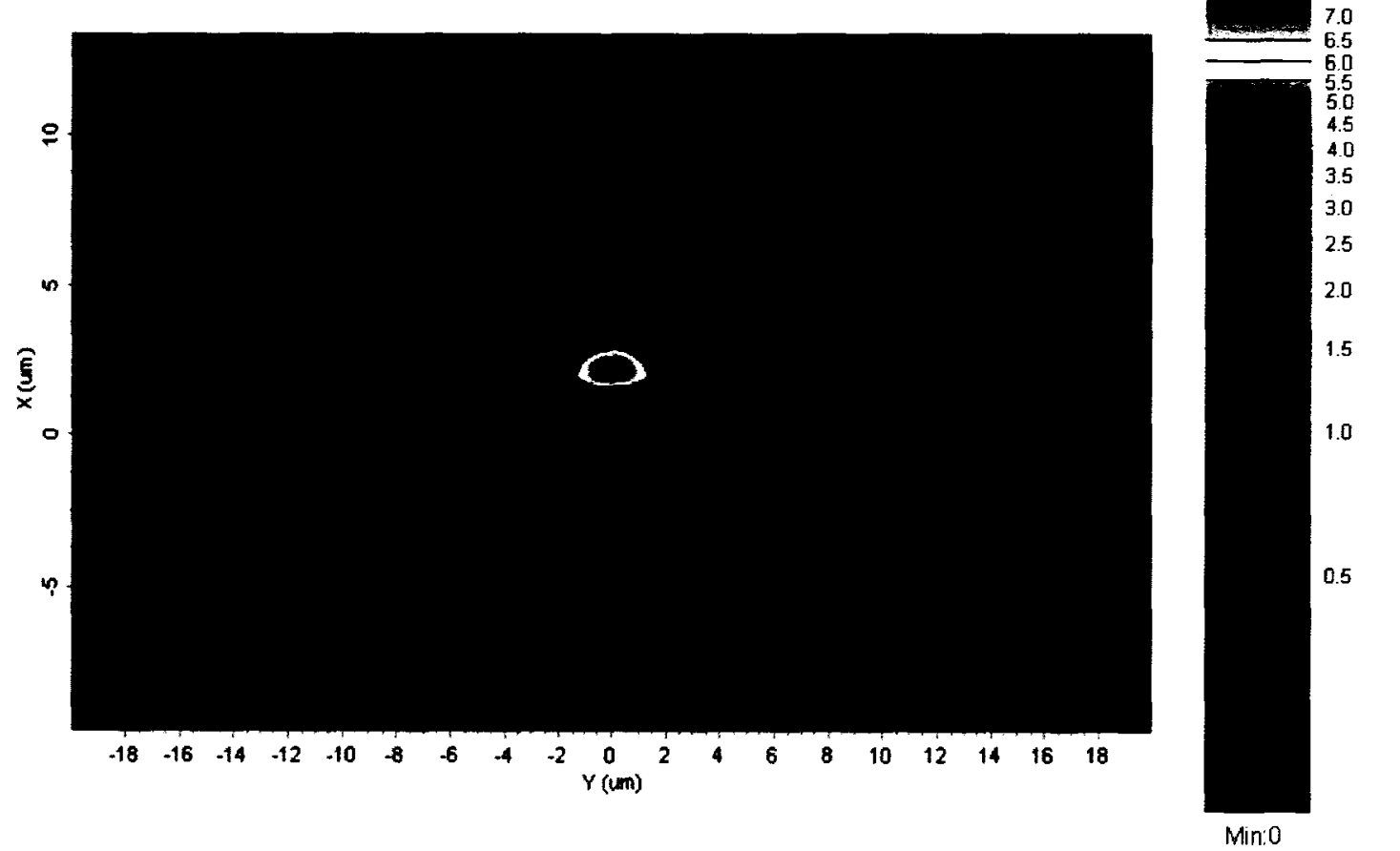

Figure 3.2: Fundamental TE waveguide mode with effective index of 3.46220 in a $2 \mu \mathrm{m}$ ridge in SEH material (2.5 $\mathrm{mm}$ silicon layer over a $1 \mu \mathrm{m}$ buried oxide layer) 


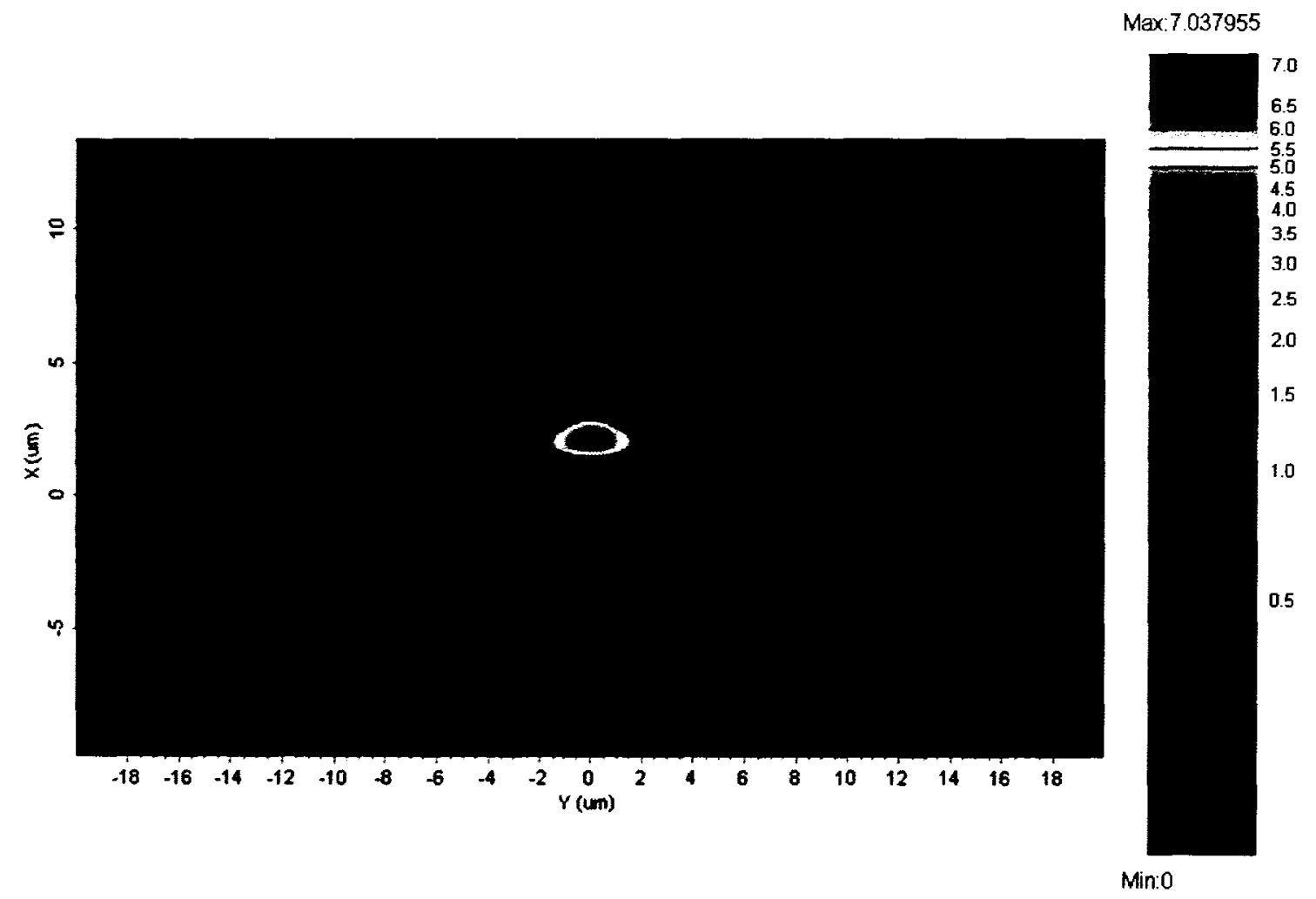

Figure 3.3: Fundamental TM waveguide mode with effective index of 3.46453 in a $2 \mu \mathrm{m}$ ridge in SEH material ( $2.5 \mu \mathrm{m}$ silicon layer over a $1 \mu \mathrm{m}$ buried oxide layer)

The next solutions of the Helmholtz equation in this $2 \mu \mathrm{m}$ ridge were found to be unconfined system modes for the TE polarization and for the TM polarization as shown in Figure 3.4 and Figure 3.5. These unconfined modes do not show any intensity beneath the rib, but rather in the slab regions surrounding the rib. This lack of another mode confined beneath the rib demonstrates that this must be a single mode waveguide.

As the width of the computational window was increased, the peak intensity in these unconfined modes was always found to be concentrated at the window edges. This suggests that the unconfined modes arise as a result of the boundary conditions imposed at the limits of the computational window and as such do not represent modes within the rib waveguide. 


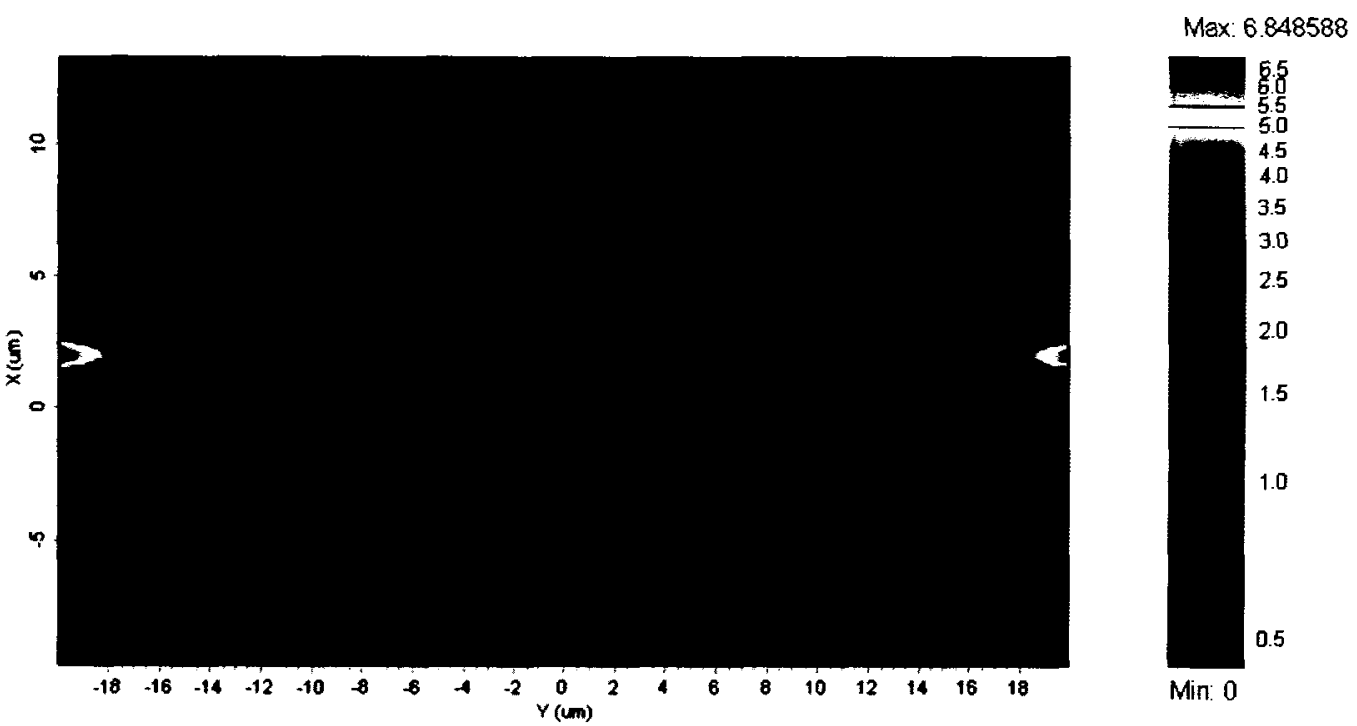

Figure 3.4: Unconfined TE mode of the LOCOS waveguide in a $2 \mu \mathrm{m}$ ridge in SEH material $(2.5 \mu \mathrm{m}$ silicon layer over a $1 \mu \mathrm{m}$ buried oxide layer)

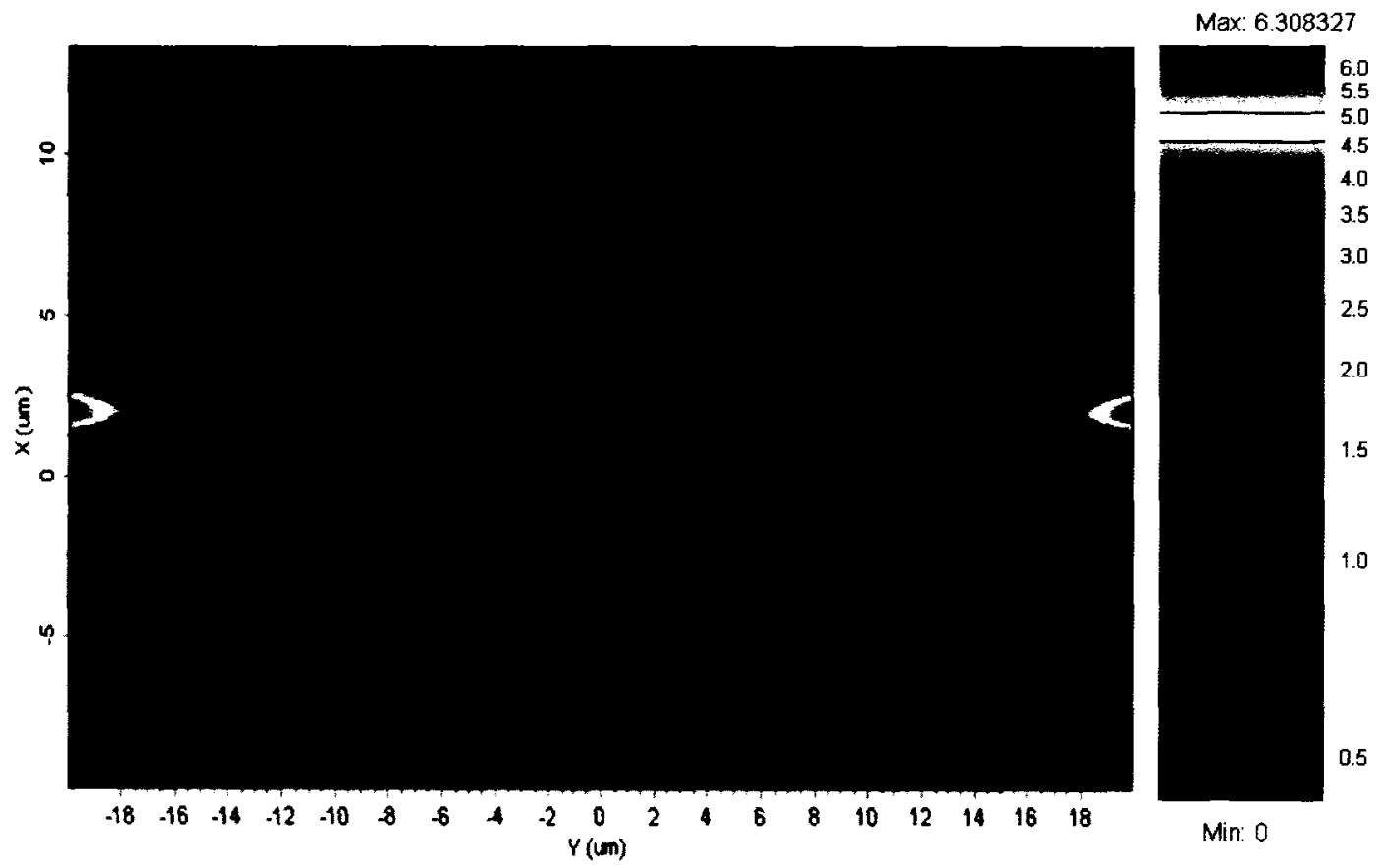

Figure 3.5: Unconfined TM mode of the LOCOS waveguide in a $2 \mu \mathrm{m}$ ridge in SEH material $(2.5 \mu \mathrm{m}$ silicon layer over a $1 \mu \mathrm{m}$ buried oxide layer)

In addition, to find the ridge width where the waveguide begins to become multimode, the second order waveguide modes were found for ridge widths ranging from $2 \mu \mathrm{m}$ to 6 $\mu \mathrm{m}$ in steps of $0.1 \mu \mathrm{m}$. The second order TE mode confined under the rib in the 
Chapter 3 Theory

waveguide was found at a width of $4.1 \mu \mathrm{m}$ and had an effective index of 3.45978 and a field profile as shown in Figure 3.6. Similarly the second order TM mode confined under the rib was found at a ridge width of $5.9 \mu \mathrm{m}$ and had an effective index of 3.46299 and the field profile shown in Figure 3.7.

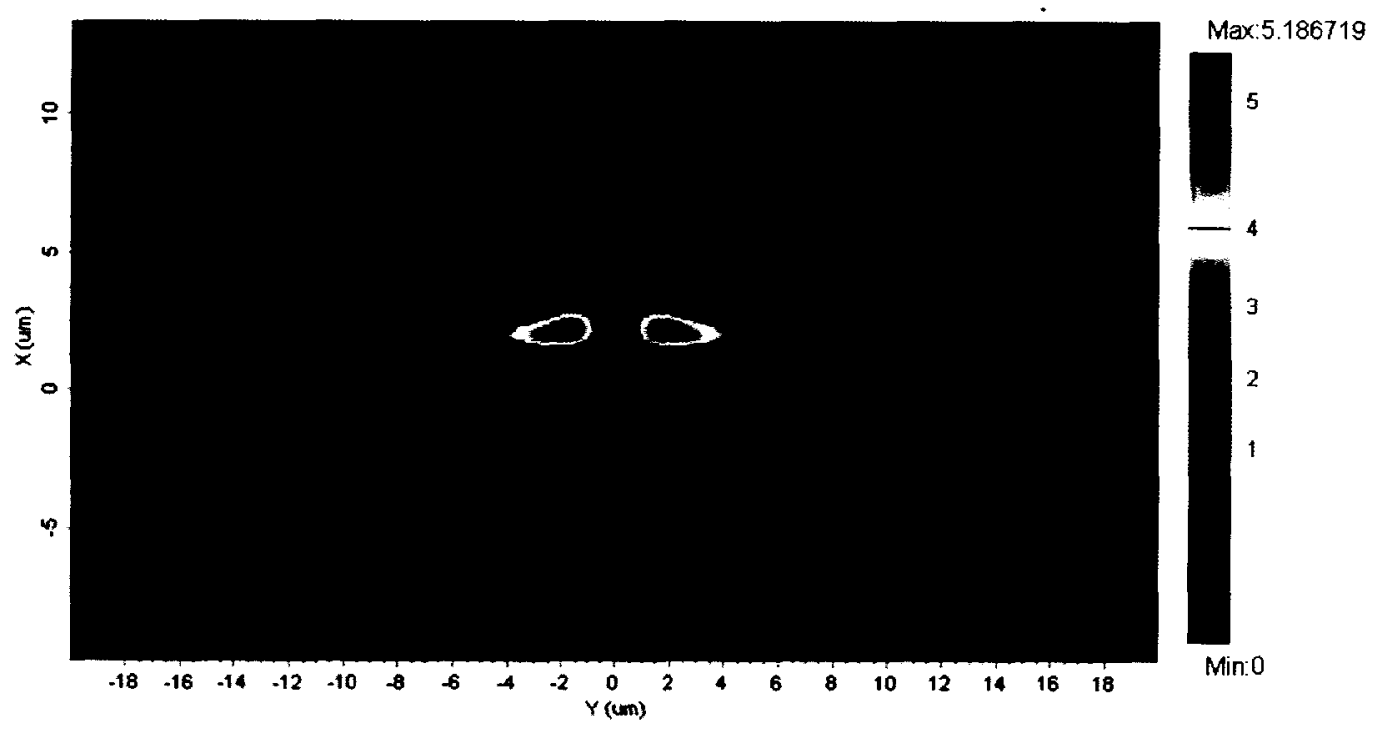

Figure 3.6: Second order TE mode of the LOCOS waveguide with an effective index of 3.45978 found at a ridge width of $4.1 \mu \mathrm{m}$ in SEH material. 


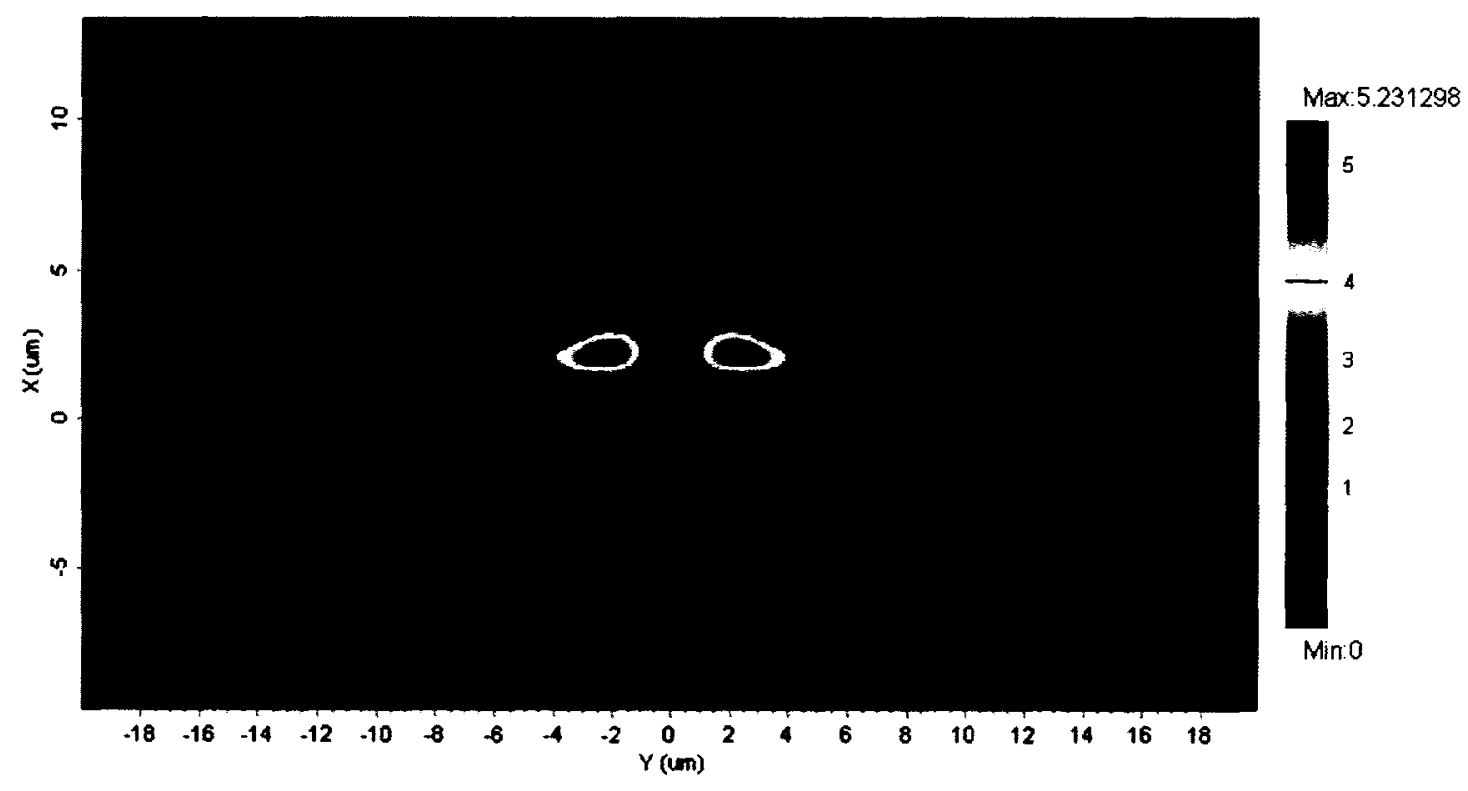

Figure 3.7: Second order TM mode of the LOCOS waveguide with an effective index of 3.46299 found at a ridge width of $5.9 \mu \mathrm{m}$ in SEH material.

Similarly, for the waveguide structures in the SOITEC wafers, the fundamental TE and TM modes of a waveguide with a $2 \mu \mathrm{m}$ wide ridge were found to have effective indices of 3.47059 and 3.47154 and mode profiles as shown in Figure 3.8 and Figure 3.9. The next solutions of the Helmholtz equation found in the waveguide with this $2 \mu \mathrm{m}$ ridge were the unconfined TE and TM modes with mode profiles as shown in Figure 3.10 and Figure 3.11 , showing the single mode nature of the $2 \mu \mathrm{m}$ rib. The first higher order TE and TM modes confined under the ridge were found when the ridge width was $7.0 \mu \mathrm{m}$ for the TE mode and $3.8 \mu \mathrm{m}$ for the TM mode. The effective indices for these higher order TE and TM modes were 3.47021 and 3.42907 respectively and their mode profiles are shown in Figure 3.12 and Figure 3.13. 


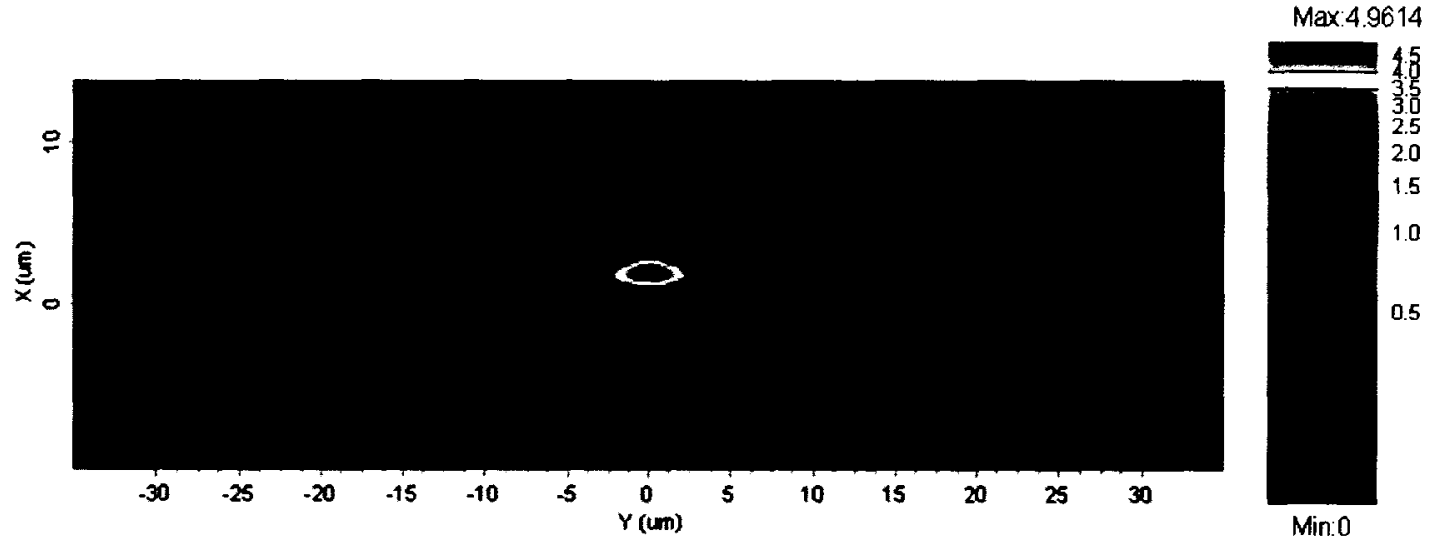

Figure 3.8: Fundamental TE waveguide mode with effective index of 3.47059 in a $2 \mu \mathrm{m}$ ridge in SOITEC material ( $3.4 \mu \mathrm{m}$ silicon layer over a $0.4 \mu \mathrm{m}$ buried oxide layer)

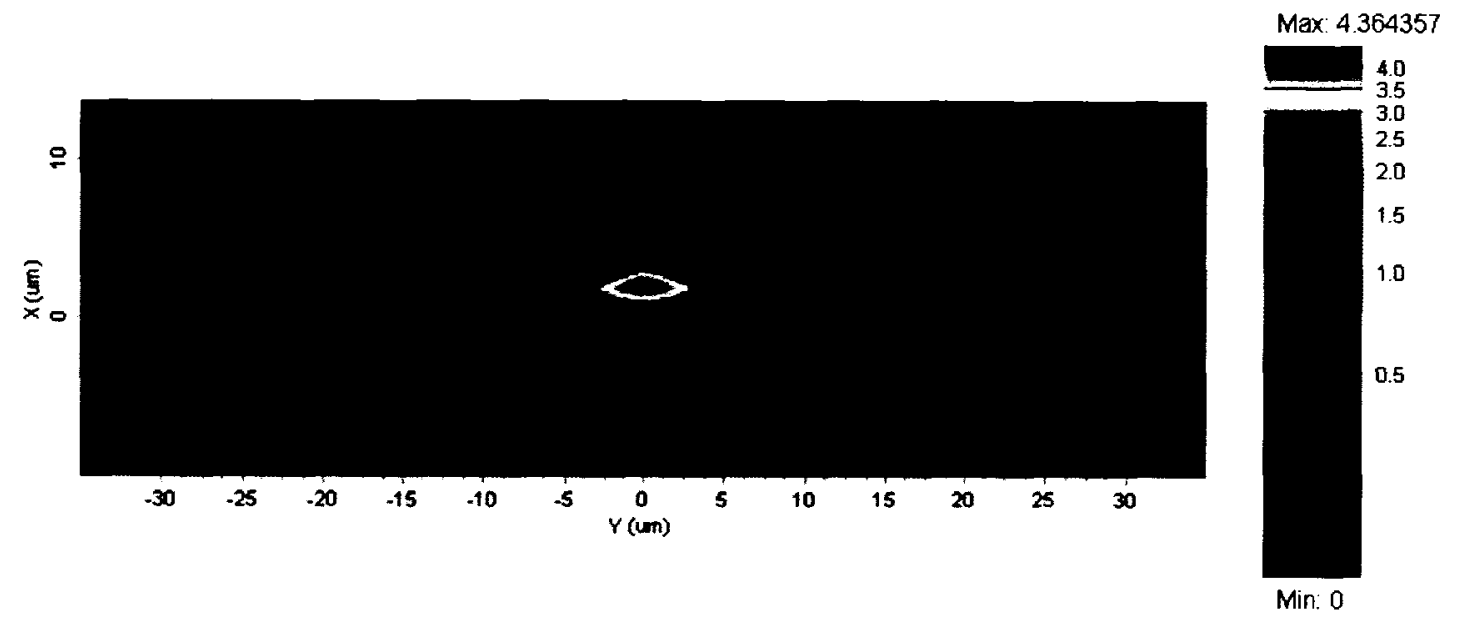

Figure 3.9: Fundamental TM waveguide mode with effective index of 3.47154 in a $2 \mu \mathrm{m}$ ridge in SOITEC material ( $3.4 \mu \mathrm{m}$ silicon layer over a $0.4 \mu \mathrm{m}$ buried oxide layer) 


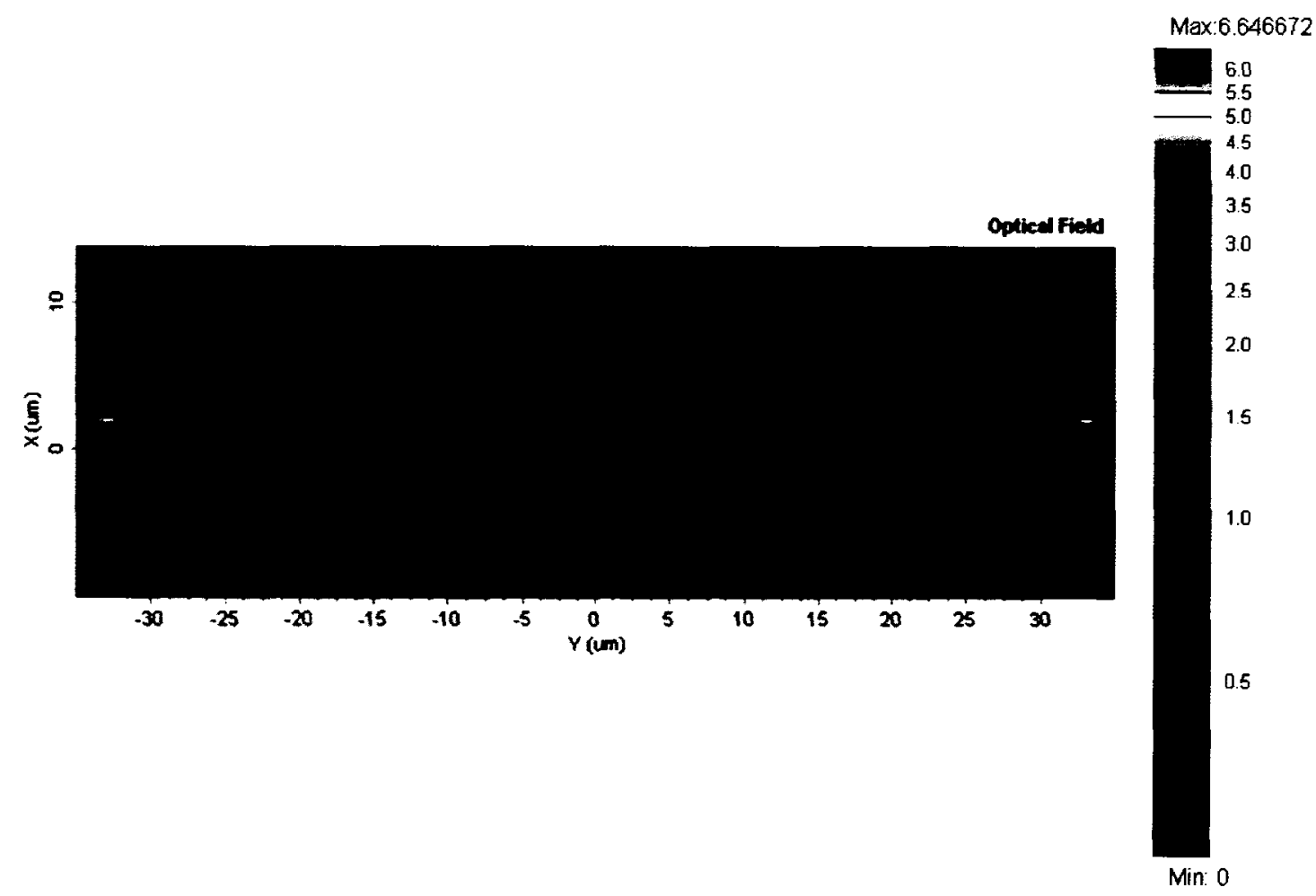

Figure 3.10: Unconfined TE mode of the LOCOS waveguide in a $2 \mu \mathrm{m}$ ridge in SOITEC material (3.4 $\mu \mathrm{m}$ silicon layer over a $0.4 \mu \mathrm{m}$ buried oxide layer)

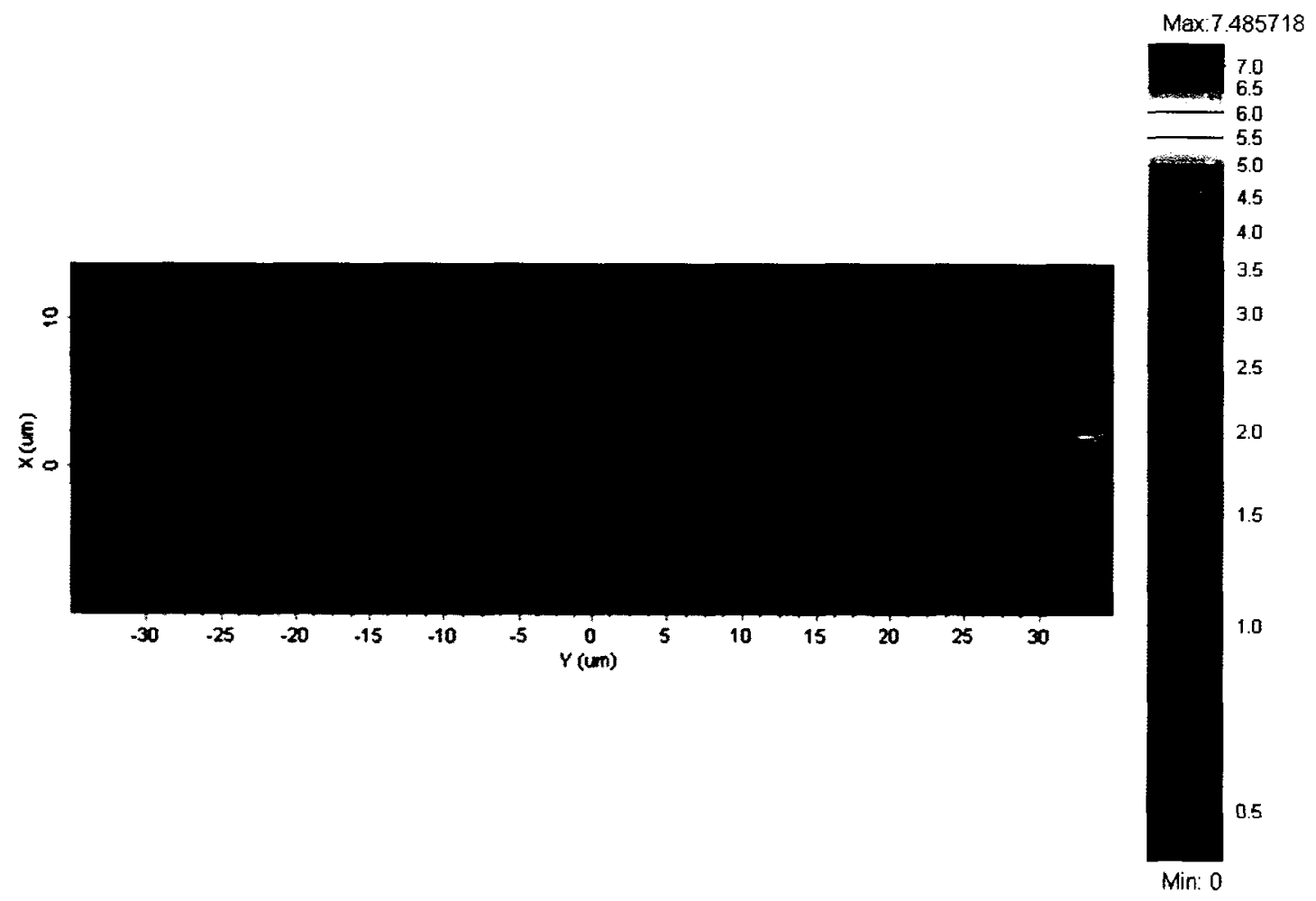

Figure 3.11: Unconfined TM mode of the LOCOS waveguide in a $2 \mu \mathrm{m}$ ridge in SOITEC material (3.4 $\mu \mathrm{m}$ silicon layer over a $0.4 \mu \mathrm{m}$ buried oxide layer) 


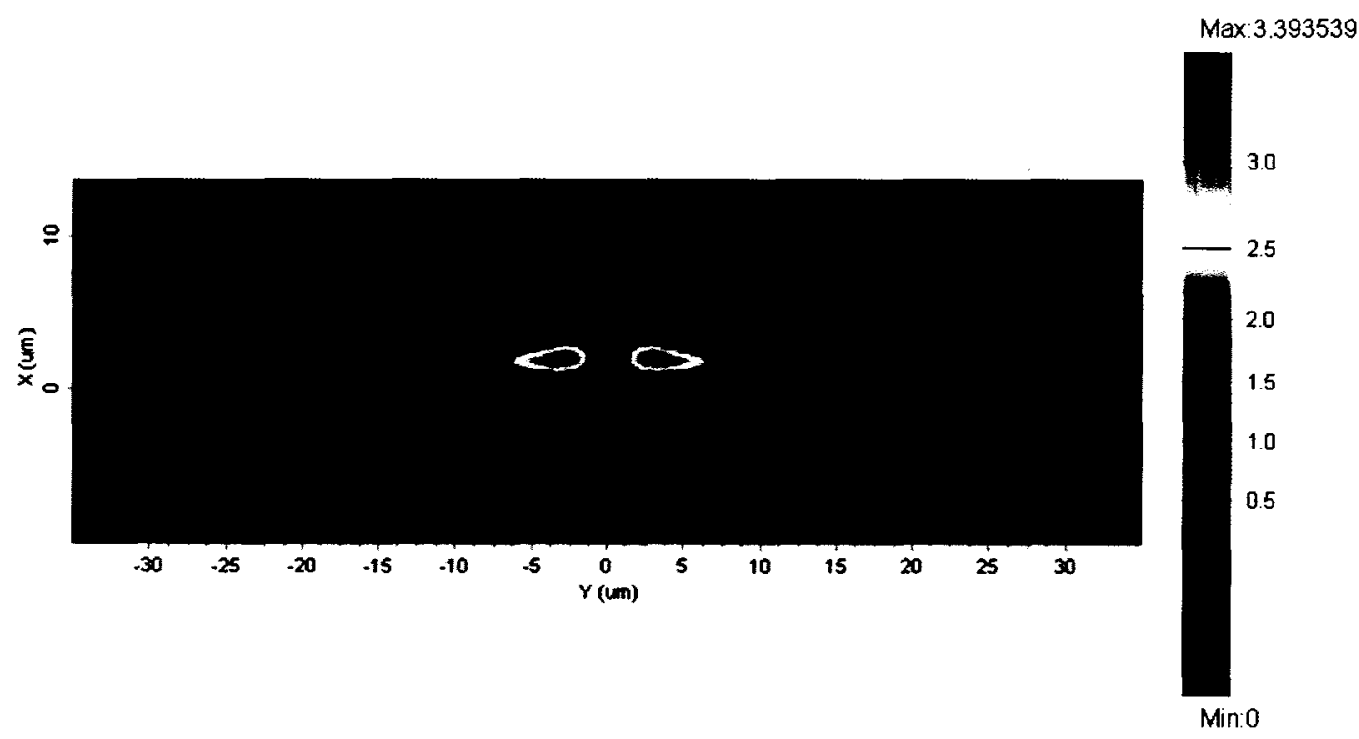

Figure 3.12: Second order TE mode of the LOCOS waveguide with an effective index of 3.47021 found at a ridge width of $7.0 \mu \mathrm{m}$ in SOITEC material.

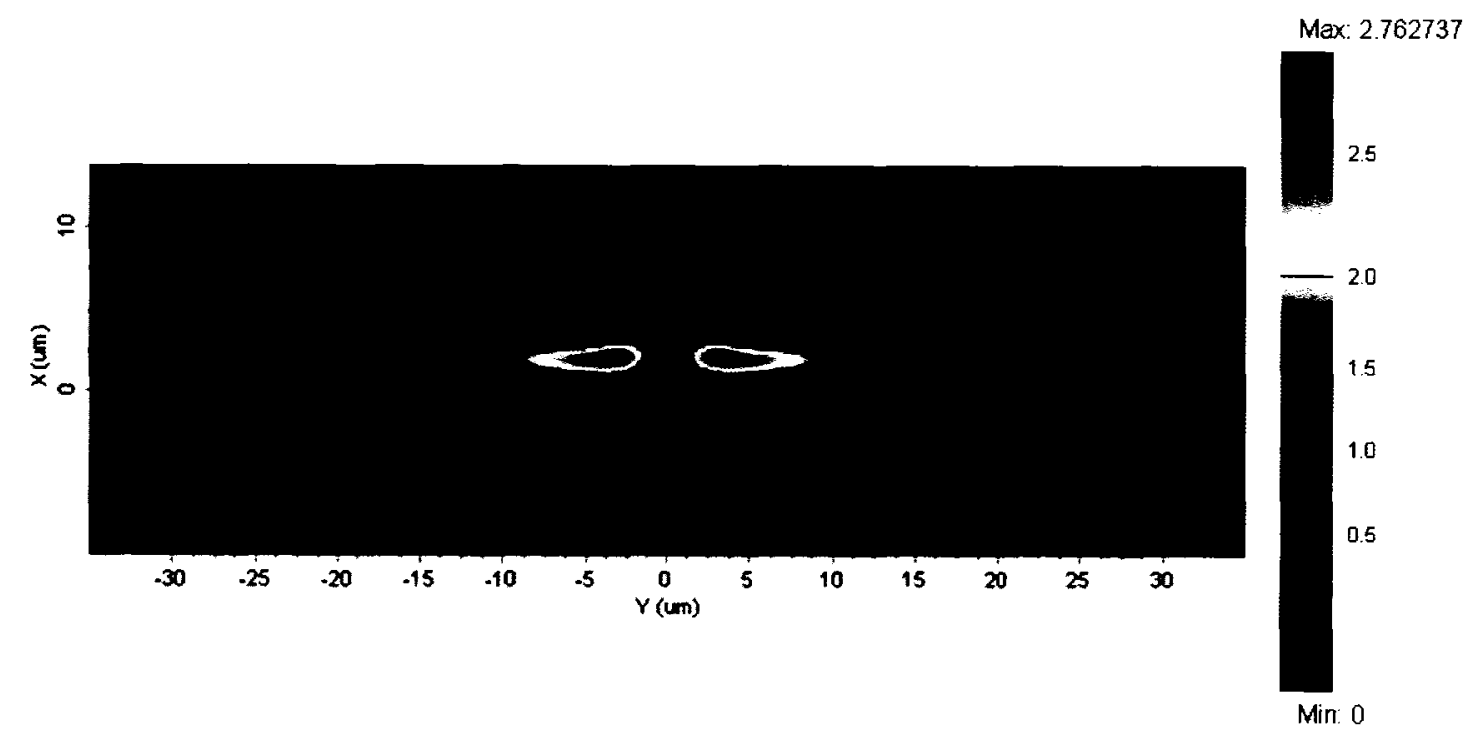

Figure 3.13: Second order TM mode of the LOCOS waveguide with an effective index of 3.42907 found at a ridge width of $7.4 \mu \mathrm{m}$ in SOITEC material.

To confirm the convergence of all of the finite difference simulations for LOCOS waveguides on both SOI substrates, the number of grid points was increased in both the horizontal and vertical directions to produce a finer mesh. The number of horizontal grid points (see Appendix A) was increased in increments of 100 to a maximum of 450 points. 
Chapter 3 Theory

Similarly, the number of vertical grid points (see Appendix A) was increased in steps of 10 within the silicon core layer and in steps of 2 in the buried oxide for each successive iteration. It was found that this affected only the 4th decimal place in the solution for the effective index and had no discernable effect on the mode profiles obtained, showing that the finite difference algorithm is stable under the simulation conditions.

\subsubsection{Mode Solving With FEMLAB}

To provide some additional confirmation for the existence of these waveguide modes, the optical modes were also analyzed by solving the Helmholtz equation with FEMLAB [Com04], a partial differential equation solver employing the finite element method as was described in section 2.5.1.

A different waveguide model from the one used with Apollo Photonic Solutions in the previous section was used for this simulation. The main differences here, as can be seen in Figure 3.14, were the extension of the oxide above the ridge to more accurately model the "bird's beak" structure produced by the LOCOS method of defining the waveguide ridge and the exclusion of the silicon substrate layer. In addition perfect electric conductor boundary conditions were imposed at each of the computational window boundaries. These conditions force the field to zero at each of the boundaries rather than forcing the slope of the field to zero as in the Apollo simulations. The simulation parameters used are shown in Table 3.3 and the refractive indices are given in Table 3.2. 


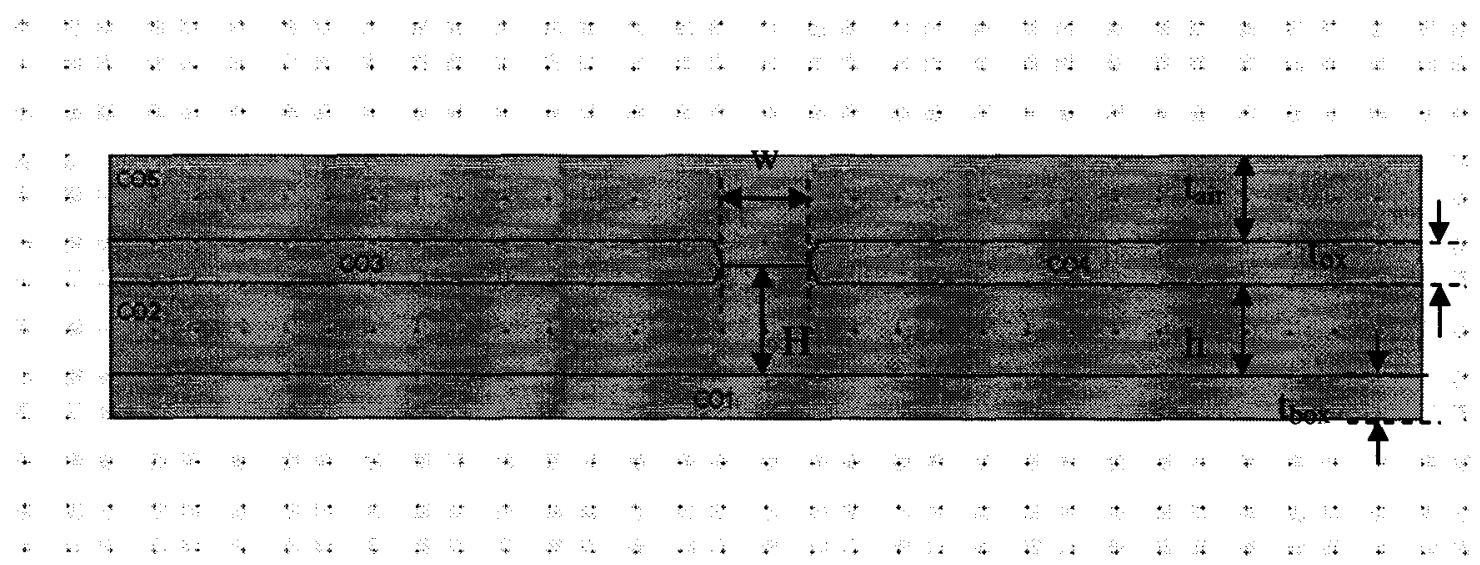

Figure 3.14: Cross-sectional view of LOCOS waveguide structure used for FEMLAB simulation. The only difference between this structure and the one in Figure 3.1 is the extension of the isolation oxide above the ridge to more accurately model the "bird's beak" extension produced by the LOCOS process.

\begin{tabular}{|l|l|l|}
\hline Name & Parameter & Value \\
\hline Ridge width & $\mathrm{w}$ & 2 to $6 \mu \mathrm{m}(2$ to $6 \mu \mathrm{m})$ \\
\hline Channel height & $\mathrm{H}$ & $2.5 \mu \mathrm{m}(3.4 \mu \mathrm{m})$ \\
\hline Slab height & $\mathrm{h}$ & $2.05 \mu \mathrm{m}(2.95 \mu \mathrm{m})$ \\
\hline Buried oxide thickness & $\mathrm{t}_{\mathrm{box}}$ & $1 \mu \mathrm{m}(0.4 \mu \mathrm{m})$ \\
\hline Field oxide thickness & $\mathrm{t}_{\mathrm{ox}}$ & $1 \mu \mathrm{m}(1 \mu \mathrm{m})$ \\
\hline Air layer thickness & $\mathrm{t}_{\mathrm{air}}$ & $1.95 \mu \mathrm{m}(1.95 \mu \mathrm{m})$ \\
\hline
\end{tabular}

Table 3.3: Simulation parameters used in FEMLAB for SEH (SOITEC) wafers

The fundamental TE and TM modes in the LOCOS waveguide with the $2 \mu \mathrm{m}$ ridge width were found to have effective indices of 3.463895 and 3.464078 respectively and mode profiles as shown in Figure 3.15 and Figure 3.16. The next TE mode of this LOCOS waveguide structure is the unconfined mode as shown in Figure 3.17. As with the Apollo simulations, this unconfined mode remains under the slab regions of the waveguide with 
Chapter 3 Theory

the maximum intensity occurring at the computational window boundary. As before, this demonstrates that these waveguides are single mode at a ridge width of $2 \mu \mathrm{m}$ in the SEH material.

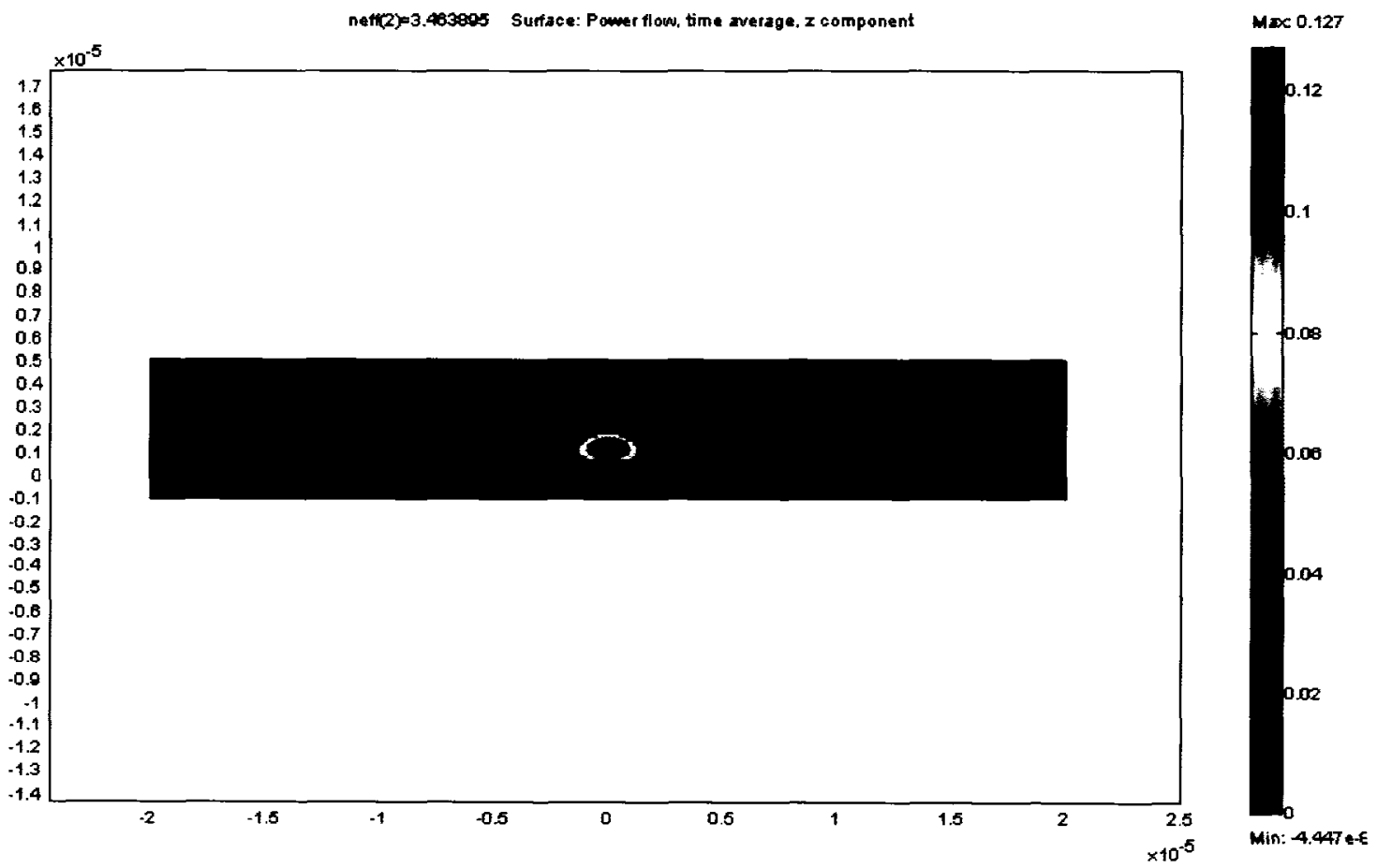

Figure 3.15: Fundamental TE mode in LOCOS waveguide with $2 \mu \mathrm{m}$ ridge width in SEH material as determined by simulation with FEMLAB. 


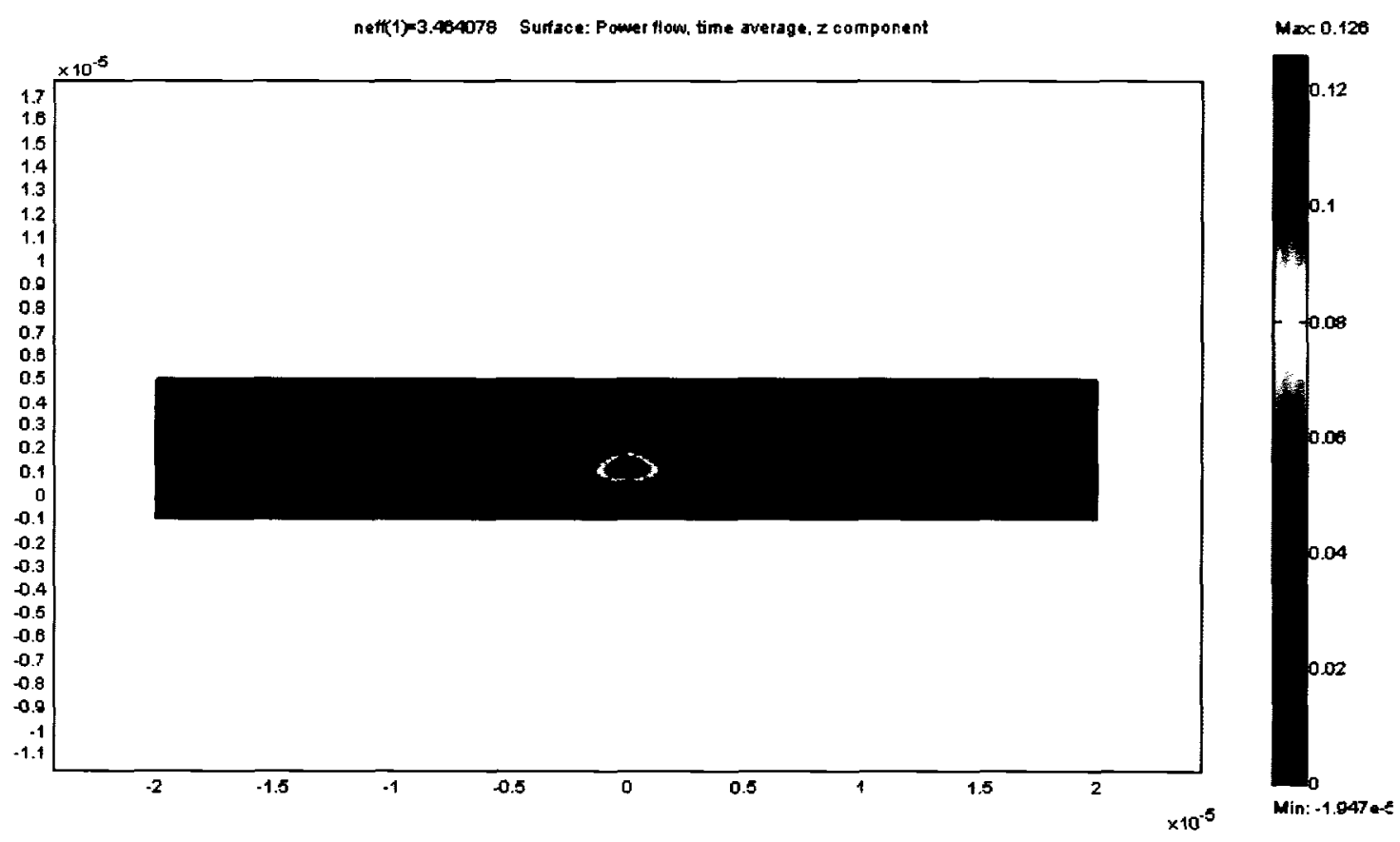

Figure 3.16: Fundamental TM mode in LOCOS waveguide with $2 \mu \mathrm{m}$ ridge width in SEH material as determined by simulation with FEMLAB.

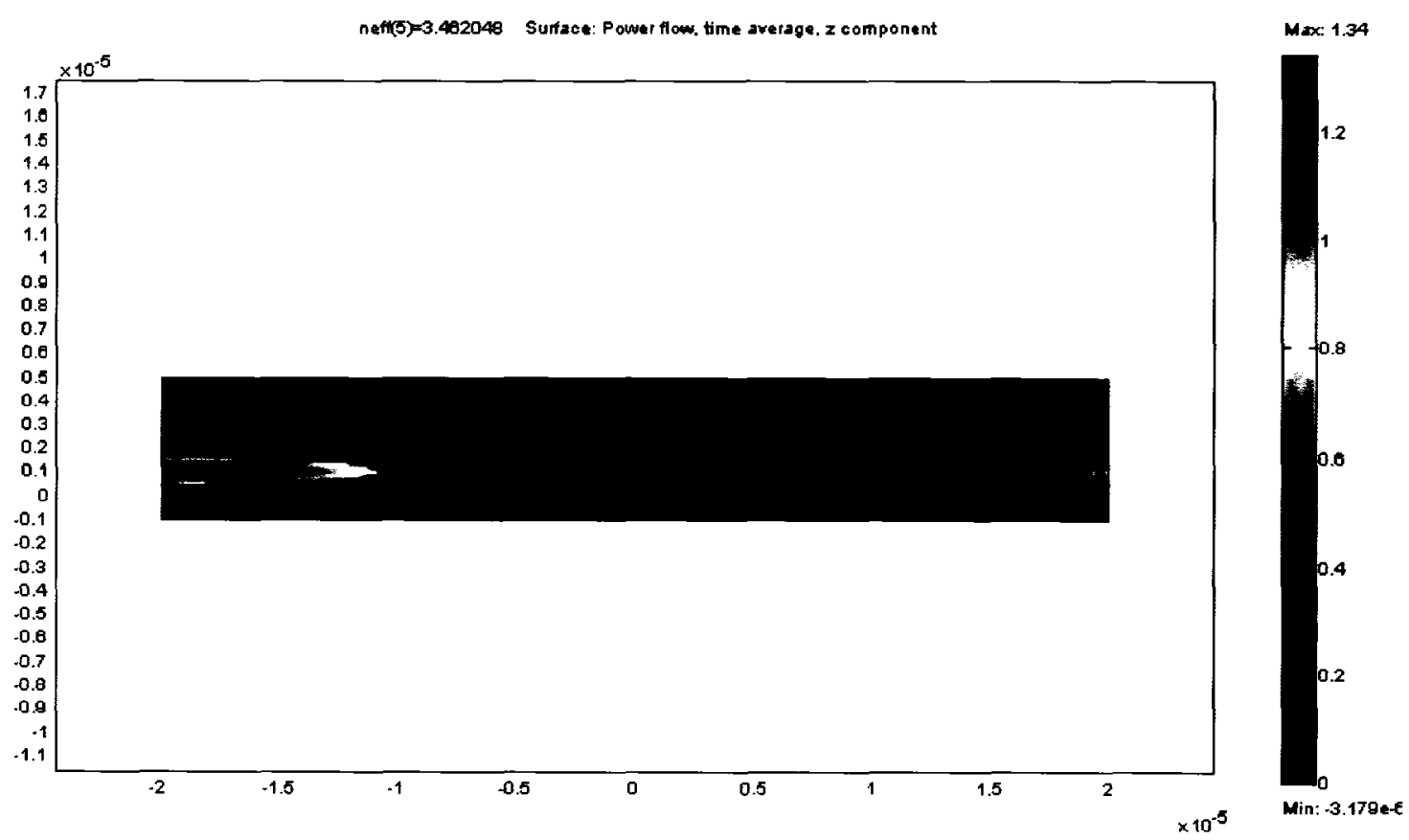

Figure 3.17: First unconfined TE mode in LOCOS waveguide with $2 \mu \mathrm{m}$ ridge width in SEH material as determined by simulation with FEMLAB.

Simulations of the same waveguide with a $2 \mu \mathrm{m}$ ridge in the SOITEC SOI material was also done with FEMLAB to confirm the existence of the confined modes and the single 
Chapter 3 Theory

mode nature of this design as shown with Apollo. In this case, the fundamental TE and

TM modes were found to have effective indices of 3.471353 and 3.471360 and mode

profiles as shown in Figure 3.18 and Figure 3.19. The first unconfined modes with TE

and TM polarizations were found to have the mode profiles shown in Figure 3.20 and

Figure 3.21 .

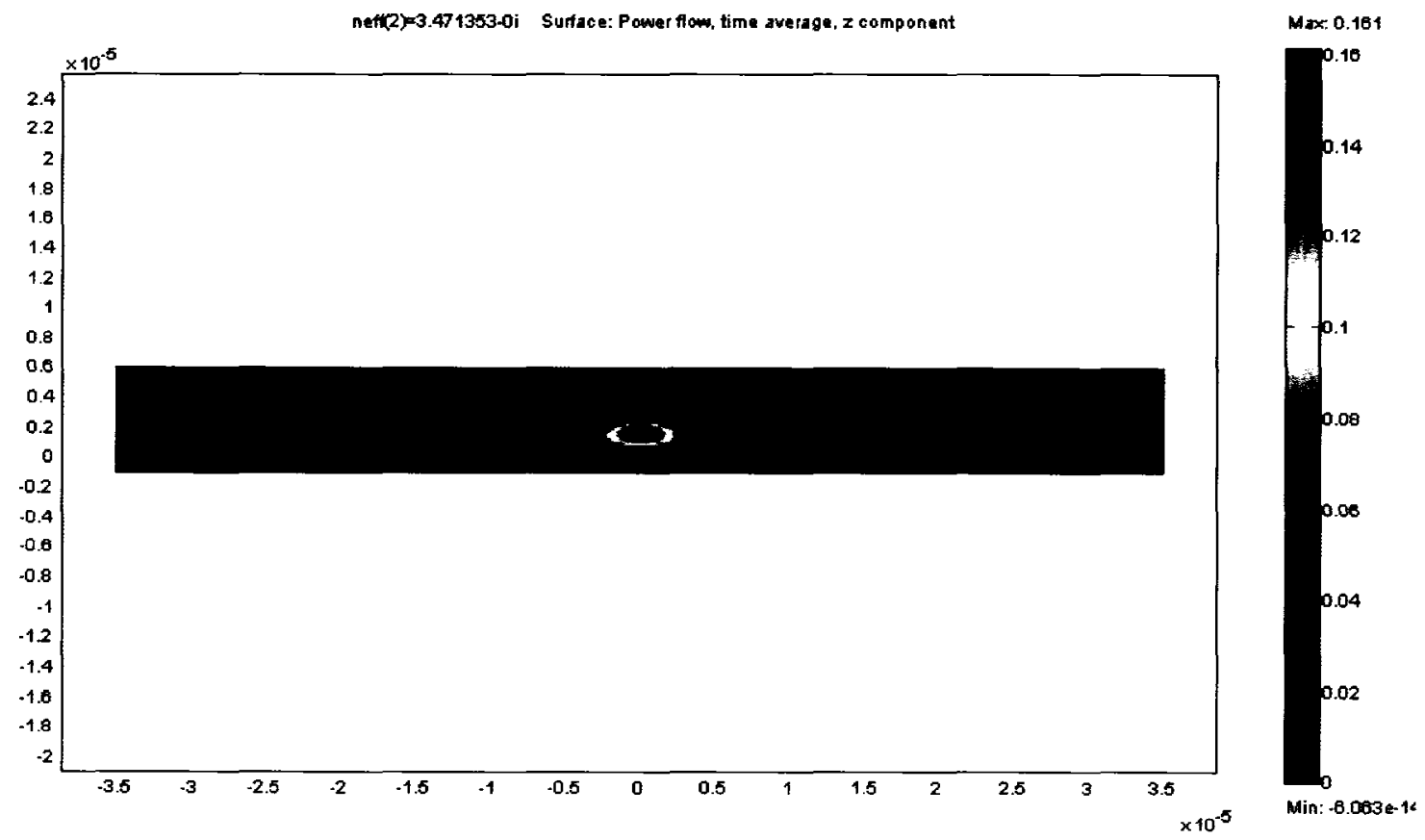

Figure 3.18: Fundamental TE mode of LOCOS waveguide with $2 \mu \mathrm{m}$ ridge in SOITEC material as determined by simulation with FEMLAB. 
Chapter 3 Theory

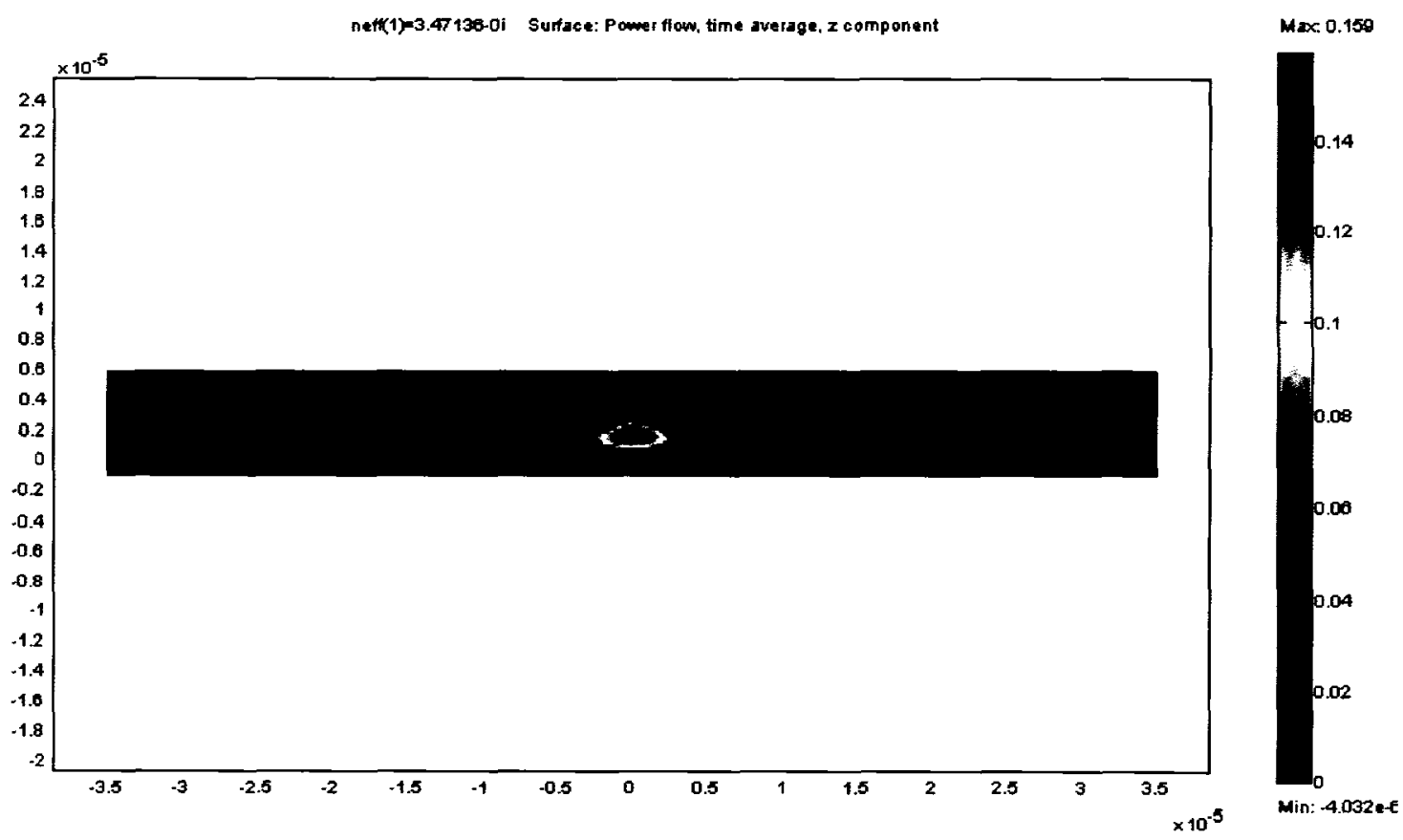

Figure 3.19: Fundamental TM mode of LOCOS waveguide with $2 \mu \mathrm{m}$ ridge in SOITEC material as determined by simulation with FEMLAB.

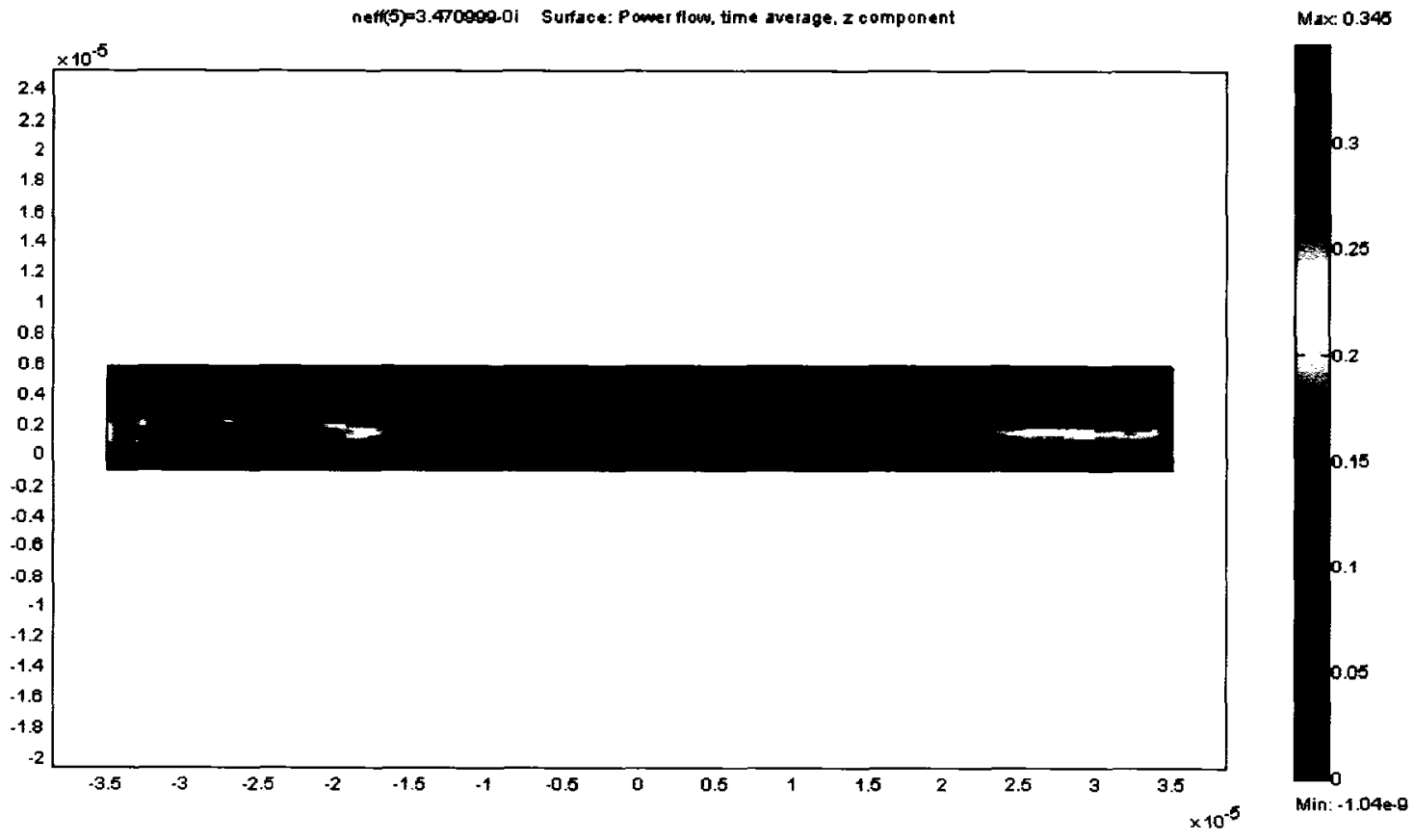

Figure 3.20: First unconfined TE mode of LOCOS waveguide with $2 \mu \mathrm{m}$ ridge in SOITEC material as determined by simulation with FEMLAB. 


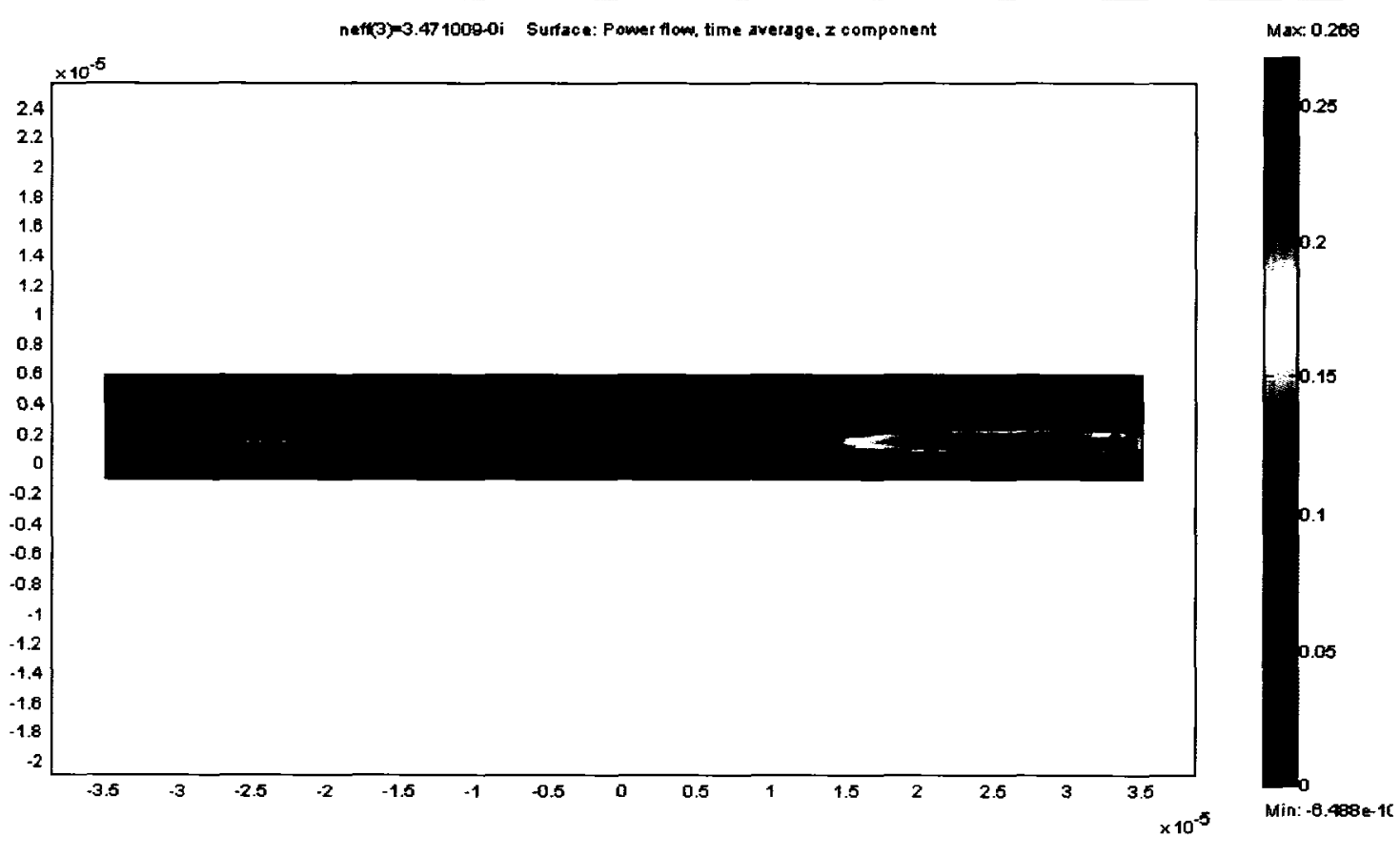

Figure 3.21: First unconfined TM mode of LOCOS waveguide with $2 \mu \mathrm{m}$ ridge in SOITEC material as determined by simulation with FEMLAB.

A similar process of mesh refinement was used on the results obtained from FEMLAB to confirm the convergence of the simulations. In this case the mesh refinement was performed only in the region surrounding the rib. This refinement resulted in a doubling of the number of mesh points in the simulation and only affected the $5^{\text {th }}$ decimal place in the effective indices of the modes obtained. Further increases in the number of mesh points was not possible as this would cause the simulator to run out of memory while computing the solutions.

Effective indices of the modes obtained with FEMLAB seem to agree with those obtained with Apollo to only the second or third decimal place. However, the relative difference between the indices of each of the modes agrees to the third decimal place which would tend to indicate that the difference observed is likely due to the difference between the models used in the FEMLAB and Apollo simulations. Single mode widths obtained in FEMLAB also agree with those obtained in Apollo. 


\subsection{Waveguide Birefringence and Sidewall Angle}

As described in [Pear00], the birefringence of a waveguide will depend upon the waveguide geometry and in particular the sidewall angle. In this study, we will look at the impact of the sidewall angle in waveguides fabricated using the LOCOS process as well as the modified forms of the LOCOS process on the waveguide birefringence.

As we saw in section 2.3, LOCOS and its modified forms give rise to a bird's beak encroachment, which results in angled sidewalls and a reduction in rib width when this process is used in forming waveguide ribs. While ridge width and etch depth are known to have a significant effect on birefringence in RIE etched rib waveguides, this effect is known to be reduced in wet-etched rib waveguides with angled sidewalls [Pear00]. Given that this birefringence plays an important role in polarization dependent loss and cross talk in complex waveguide devices, the effect of the angled sidewalls in waveguides fabricated by LOCOS processes on the birefringence should be characterized.

Optical mode simulations with Apollo were used to find the effective indices of the TE and TM polarized optical modes in waveguides with sidewall angles which approximate the slope obtained from the bird's beak encroachments produced by the LOCOS and PBL processes studied in this project (see section 3.4). The difference in effective indices is the birefringence. In this study, the birefringence was defined as

$$
\Delta n_{\text {eff }}=n_{\text {effTM }}-n_{\text {effTE }}
$$

For comparison, a waveguide with vertical sidewalls and an oxide trench in the slab region was also included in this study. The waveguide width was swept from $2 \mu \mathrm{m}$ to $6 \mu \mathrm{m}$ for each structure in both the SEH and SOITEC SOI substrates, the effective indices were found for both the TE and TM polarized modes and the birefringence was computed 
Chapter 3 Theory

according to the definition in (3.1). The results obtained for these structures are

summarized in Figure 3.22 and Figure 3.23.

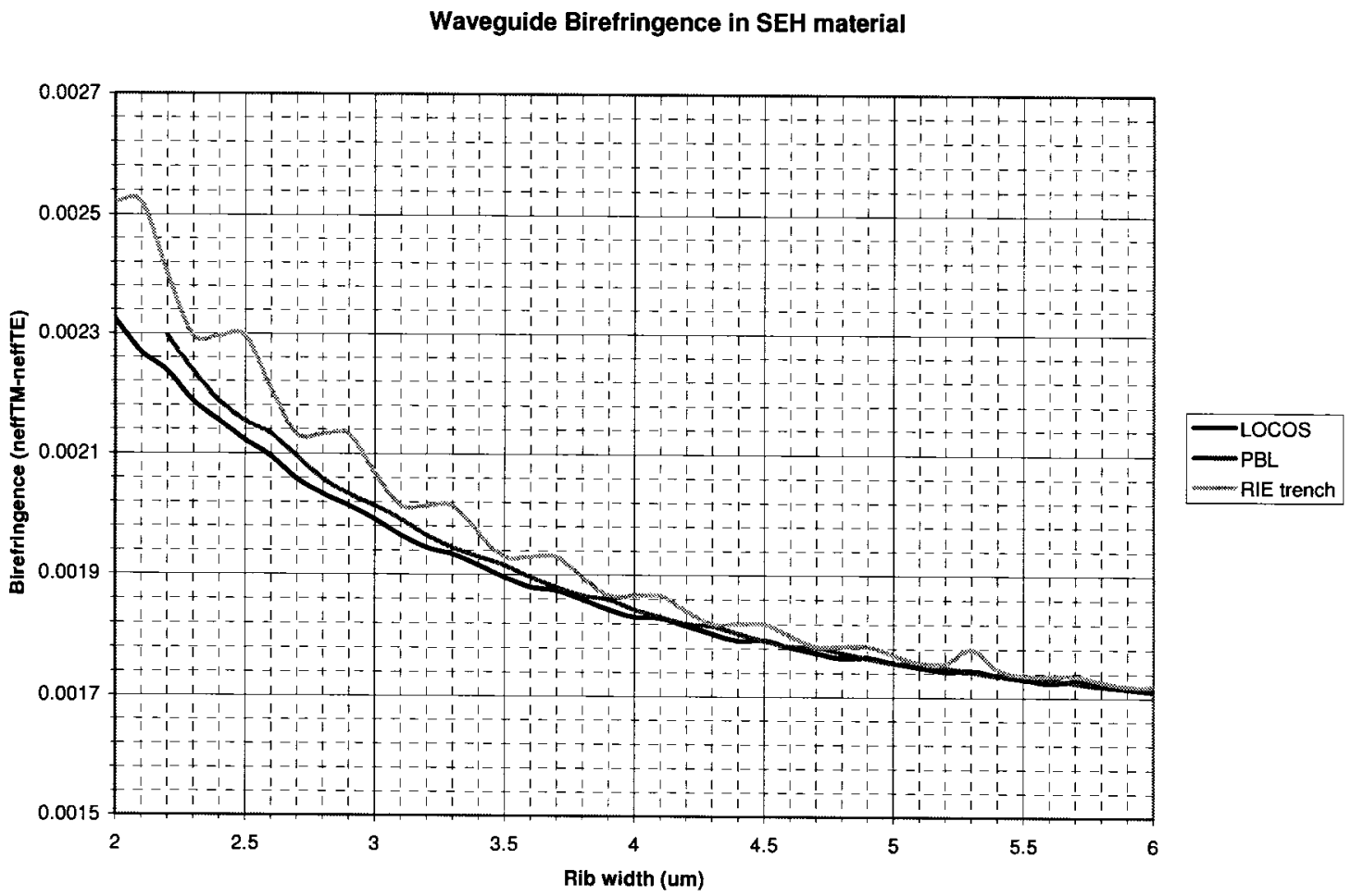

Figure 3.22: Birefringence as a function of rib width for LOCOS, PBL, and RIE trench waveguides in SEH substrate 
Waveguide BIrefringence in SOITEC material

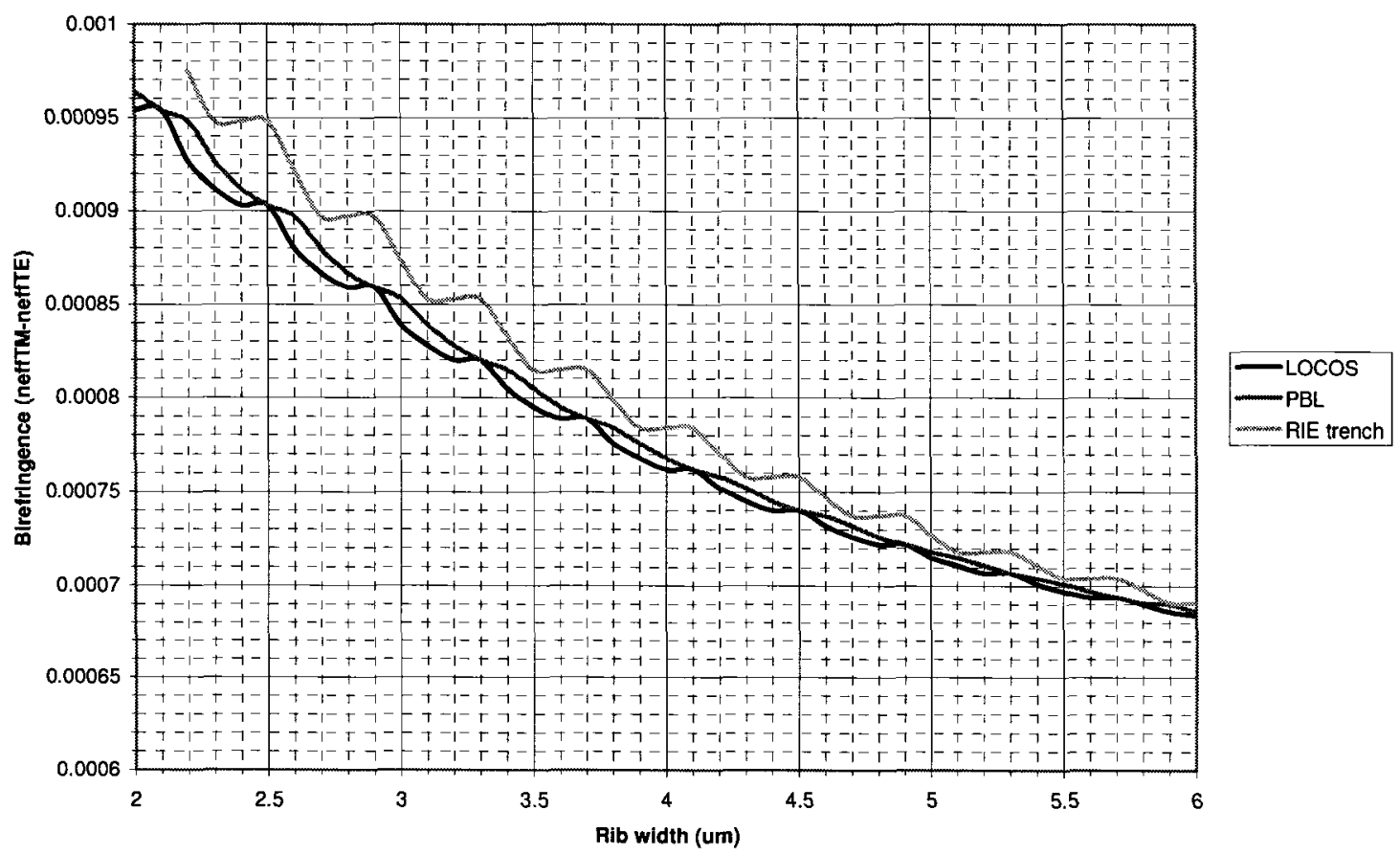

Figure 3.23: Birefringence as a function of rib width for LOCOS, PBL, and RIE trench waveguides in SOITEC substrate.

As can be seen from these results, the waveguides formed with the conventional LOCOS process used for device isolation in the Carleton nMOS process (see section 3.4.1) show the least birefringence due to their more significant sidewall slope. Other waveguide processes with steeper sidewall slopes show an increased birefringence, which can cause unwanted polarization dependent effects in complex waveguide devices. We also see that as with the chemically etched waveguides in [Pear00], the trend toward little variation in birefringence with a change in the rib width of the waveguide seems to hold.

This study also reveals that the silicon film thickness seems to play a role in the birefringence of the waveguide devices, with the birefringence being reduced with increasing silicon film thickness. 
Chapter 3 Theory

We can see that there is not much of an advantage to using the more advanced LOCOS isolation techniques such as SILO and PBL, and this may in fact be detrimental in complex waveguide devices in which polarization dependent effects are to be avoided.

Another effect we can see from these results (Figure 3.22 and Figure 3.23) is the "ripple" in some of the birefringence curves, particularly those for the vertical-walled RIE trench structure. In an ideal simulation, we would expect that these curves would be continuous rather than displaying this "ripple" as the birefringence depends upon the effective mode index which varies continuously with waveguide width. By increasing the number of grid points in both the horizontal and the vertical directions, it was found that the magnitude of the "ripple" could be reduced and even eliminated. This would indicate that the "ripple" is an artefact of the simulation resulting from grid spacing and its effect on the effective indices obtained.

\subsection{Beam propagation in Unbalanced Mach-Zehnder Interferometer}

In a Mach-Zehnder Interferometer (MZI), the light is passed through a splitter such that the power is divided evenly between the two output branches. A phase shift is applied to the light in one branch and the light is recombined into a single branch by being passed through the two branches of a second splitter structure and collected at the single output. The two main forms of the MZI are the balanced device in which both branches have the same physical length and the phase shift is obtained by a thermo-optic, electro-optic, or free carrier plasma effect or the unbalanced device in which the phase shift is due to the additional physical length of one branch compared to the other. 
Chapter 3 Theory

In this study, we are using the beam propagation modelling features of Apollo to simulate the propagation of light at wavelengths of $1500-1600 \mathrm{~nm}$ through an unbalanced device (see Figure 3.24). Due to the difference in the physical length between the two arms of this unbalanced MZI, light propagating in each branch will arrive out of phase at the second splitter used to recombine the waves. As a result, in general, the light at the output will be attenuated due to the partially destructive interference of the two light waves with different phase. The magnitude of the attenuation observed in the MZI output will show a wavelength dependence due to the relationships between the phase shift in the arms of the MZI and the periodicity of the light wave.

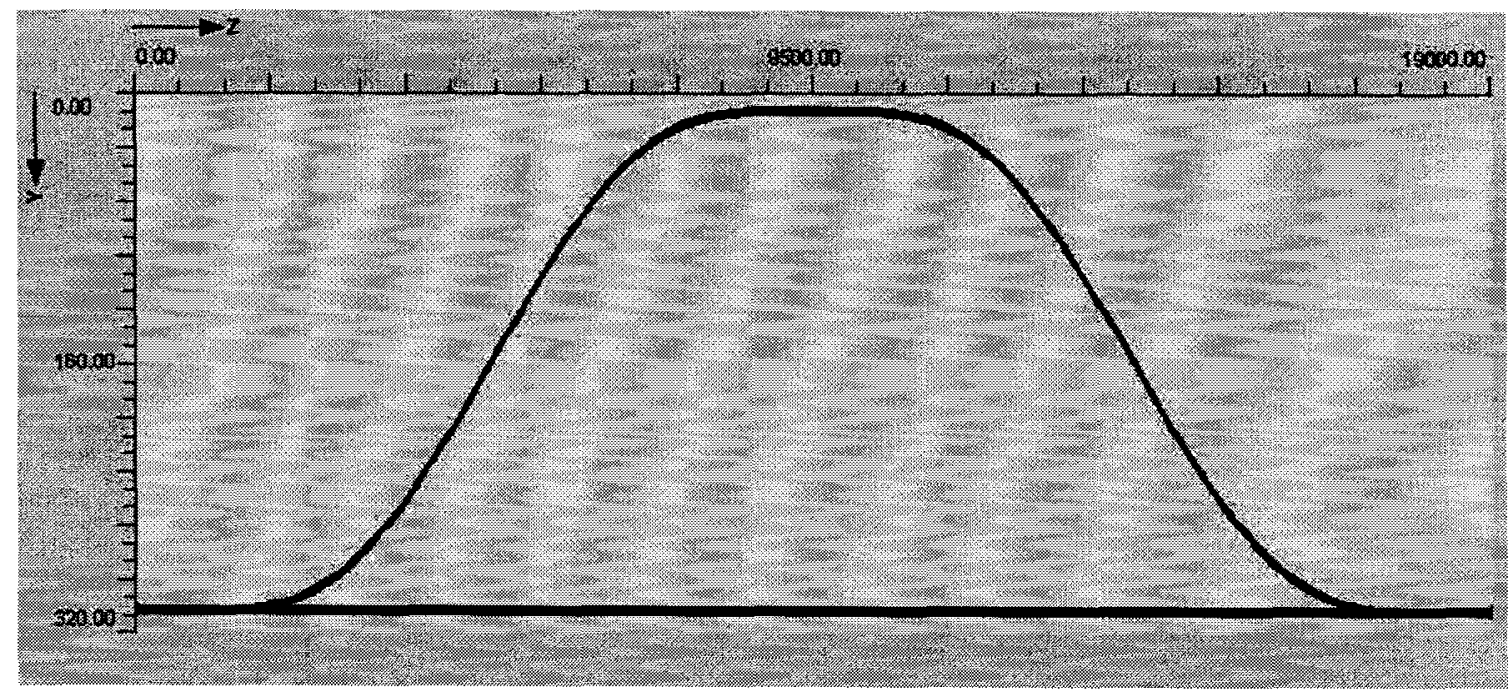

Figure 3.24: Unbalanced MZI device with a path length difference of $16.8577 \mu \mathrm{m}$ constructed with LOCOS waveguides with a $4 \mu \mathrm{m}$ ridge width.

The resulting attenuation spectrum obtained from simulation of this device with both TE (X) and TM (Y) polarizations is shown in below. As can be seen from Figure 3.25, the difference between the maximum and the minimum attenuation produced or the modulation depth is $12.8 \mathrm{~dB}$ for the TE polarization and $12.4 \mathrm{~dB}$ for the TM polarization. The difference in wavelengths for the peak power for each polarization is due to the 
Chapter 3 Theory

birefringence of the waveguide and partially accounts for the difference in modulation depths observed.

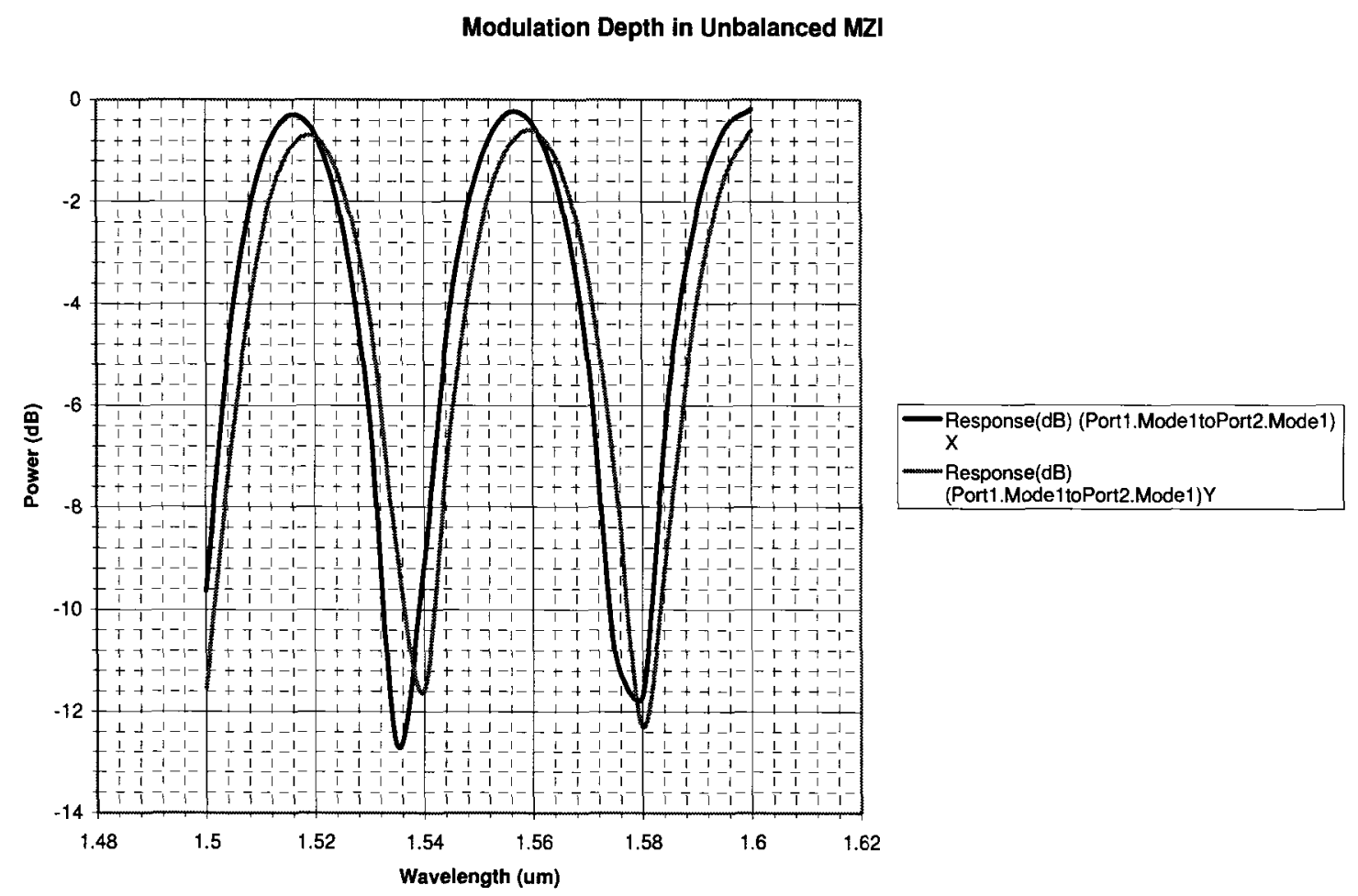

Figure 3.25: Attenuation from constructive and destructive interference in the MZI device of Figure 3.24 with TE (X) and TM (Y) polarized light.

Although, this is an ideal simulation where a perfect extinction (infinite on the $\mathrm{dB}$ scale) should be observed, practical limitations on the sampling resolution due to computational time and memory requirements prevent this perfect extinction from actually being observed. The difference in the observed modulation depths in the unbalanced MZI for the TE and the TM polarizations can easily be explained by the combined effect of the sampling resolution limits and the birefringence-induced shift in wavelength for the peak power. 
Chapter 3 Theory

\subsection{Process Simulation}

This section will cover the simulations of the conventional LOCOS process flow completed with TSUPREM-4 as well as the simulations of two modified LOCOS process flows: Polysilicon Buffered LOCOS (PBL) and Sealed-Interface Local Oxidation (SILO). Details of these manufacturing processes as well as cross-section plots of the waveguide structures produced and oxidation stresses in the structures will also be covered for each of these processes.

All of these process flows were simulated to take place on an SOI wafer with a p-type $5 \mu \mathrm{m}$ silicon layer with a (100) orientation over a $1 \mu \mathrm{m}$ buried oxide on a p-type silicon wafer. The boron concentration used for the silicon wafer and silicon film was $1 \times 10^{15} \mathrm{~cm}^{-3}$ which roughly corresponds to $10 \Omega \mathrm{cm}$ resistivity. While these are not the silicon film and buried oxide thicknesses and the silicon resistivity that were actually used for either the SEH or SOITEC wafers, the change in optical properties would be negligible although the exact mode profile obtained may differ due to the change in ridge height and slab height obtained with other silicon film thicknesses. The buried oxide thickness change will have little impact as the $1 \mu \mathrm{m}$ thickness used here exceeds the 0.4 $\mu \mathrm{m}$ lower limit to prevent radiation loss into the substrate [Reed04].

To save on computational time and memory for storing the final structure, only half of the waveguide structure was simulated as it is symmetric about the centre of the waveguide ridge. This simplification captures all of the required 2-dimensional topographical information. The full structure could easily be reproduced for plotting purposes by reflecting the structure about the line passing vertically through the centre of the waveguide ridge. 
Chapter 3 Theory

In addition, only the first $2.5 \mu \mathrm{m}$ of the field region was included in the simulation and the rib width used on the mask was selected as $5 \mu \mathrm{m}$ such that the overall simulation region was only $5 \mu \mathrm{m}$ wide to provide additional savings of computational time and memory. Only the first $5 \mu \mathrm{m}$ microns of the depth of the silicon substrate wafer was included in the simulation to save on memory as it is highly unlikely that any process would affect the silicon substrate at a greater depth and, in fact, an even shallower depth into the silicon substrate could have been used.

\subsubsection{Conventional Local Oxidation of Silicon (LOCOS) Process}

Representative of many early LOCOS processes with its thick pad oxide, thin nitride layer and long bird's beak encroachment, the Carleton nMOS process begins with the thermal oxidation of the SOI wafer at $1100{ }^{\circ} \mathrm{C}$ for 11 minutes to produce roughly $50 \mathrm{~nm}$ of pad oxide on top of the silicon film. Following this, an $820^{\circ} \mathrm{C}$ deposition of silicon nitride was used to produce an $80 \mathrm{~nm}$ film to mask the field oxidation. These layers were etched to leave a $5 \mu \mathrm{m}$-wide LOCOS stack to mask the field oxidation for the waveguide ridge. Subsequently, a three stage field oxidation was carried out at $1100^{\circ} \mathrm{C}$ to produce the $1 \mu \mathrm{m}$ field oxide necessary to achieve the $0.45 \mu \mathrm{m}$ reduction in the height of the silicon layer needed to produce the slab region surrounding the rib.

The cross-section of the device structure produced with this process is shown in Figure 3.26. The compression and tension stresses within the structure accumulated during the oxidation process are shown in Figure 3.27 and Figure 3.29 respectively. 


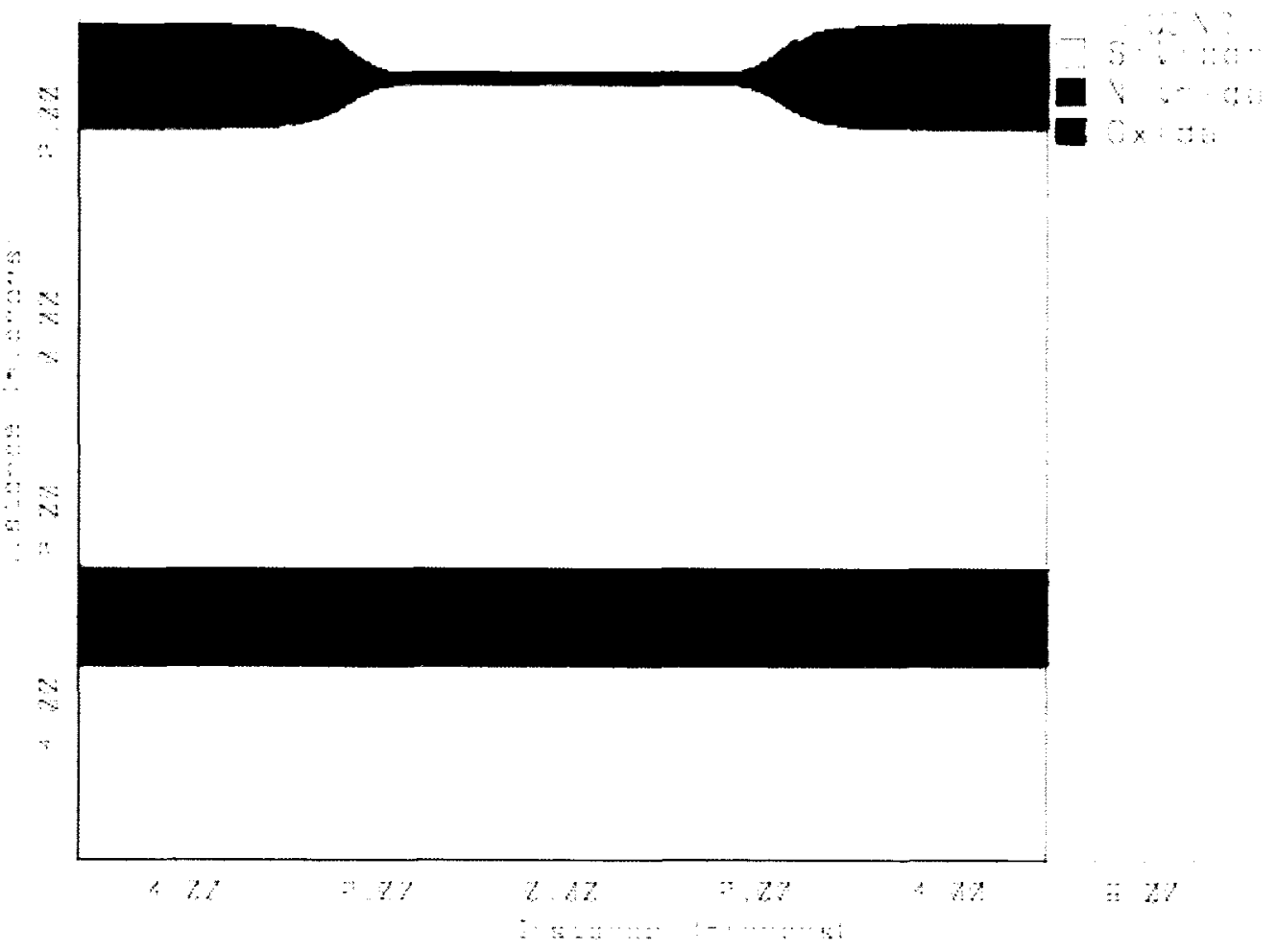

Figure 3.26: Waveguide device cross-section produced using conventional LOCOS process used for isolation in the nMOS process in use in the Carleton University Microfabrication Facility. 


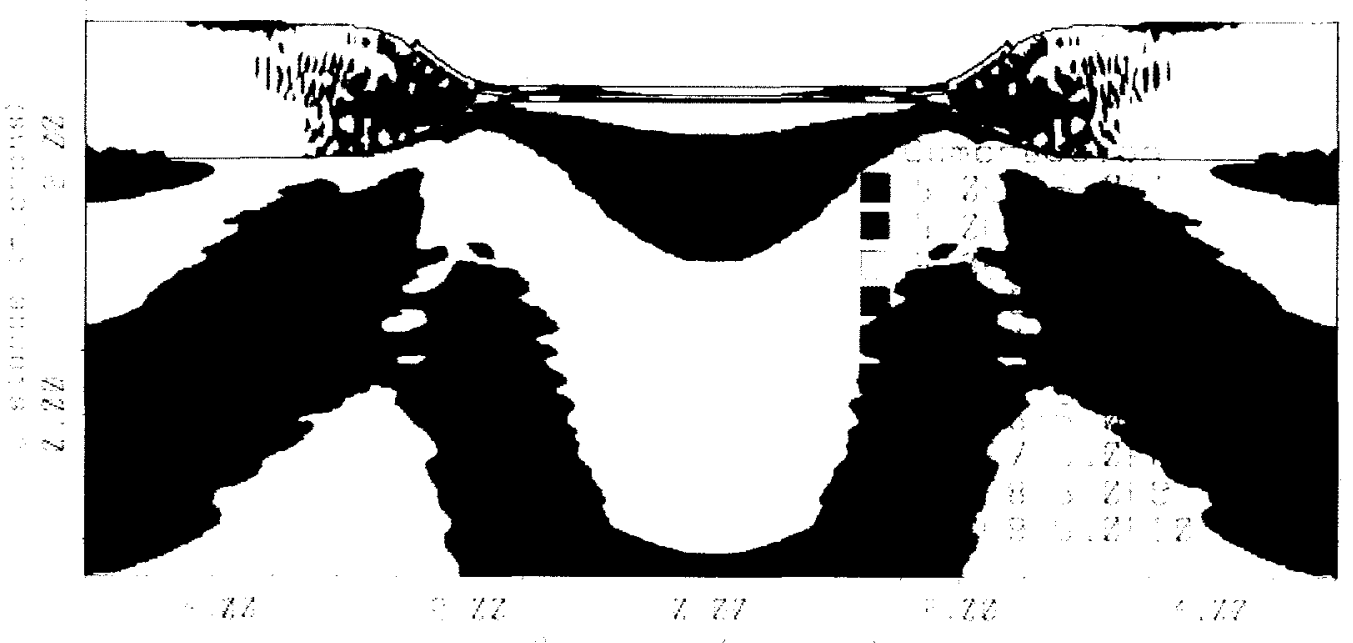

Figure 3.27: Compressive stress $\sigma_{x x}\left(\mathrm{dyn} / \mathrm{cm}^{2}\right)$ due to oxidation in LOCOS waveguide structure.

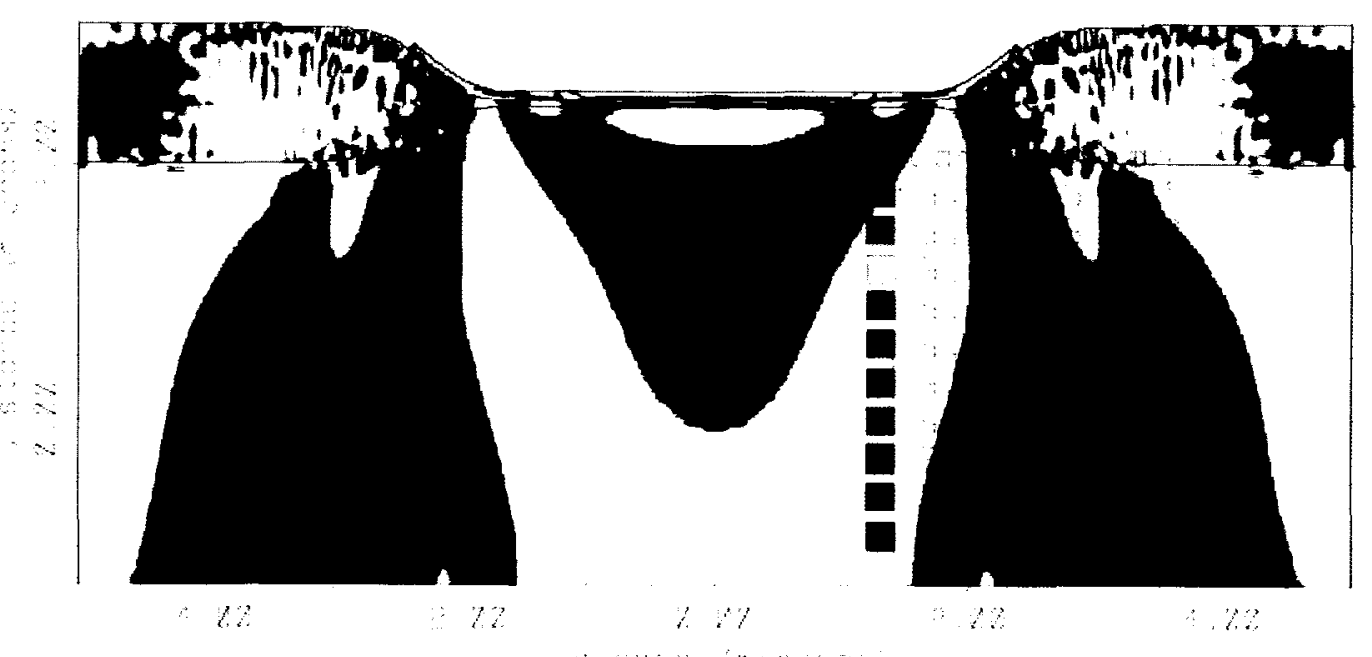

Figure 3.28: Compressive stress $\sigma_{y y}\left(\mathrm{dyn} / \mathrm{cm}^{2}\right)$ due to oxidation in LOCOS waveguide structure. 


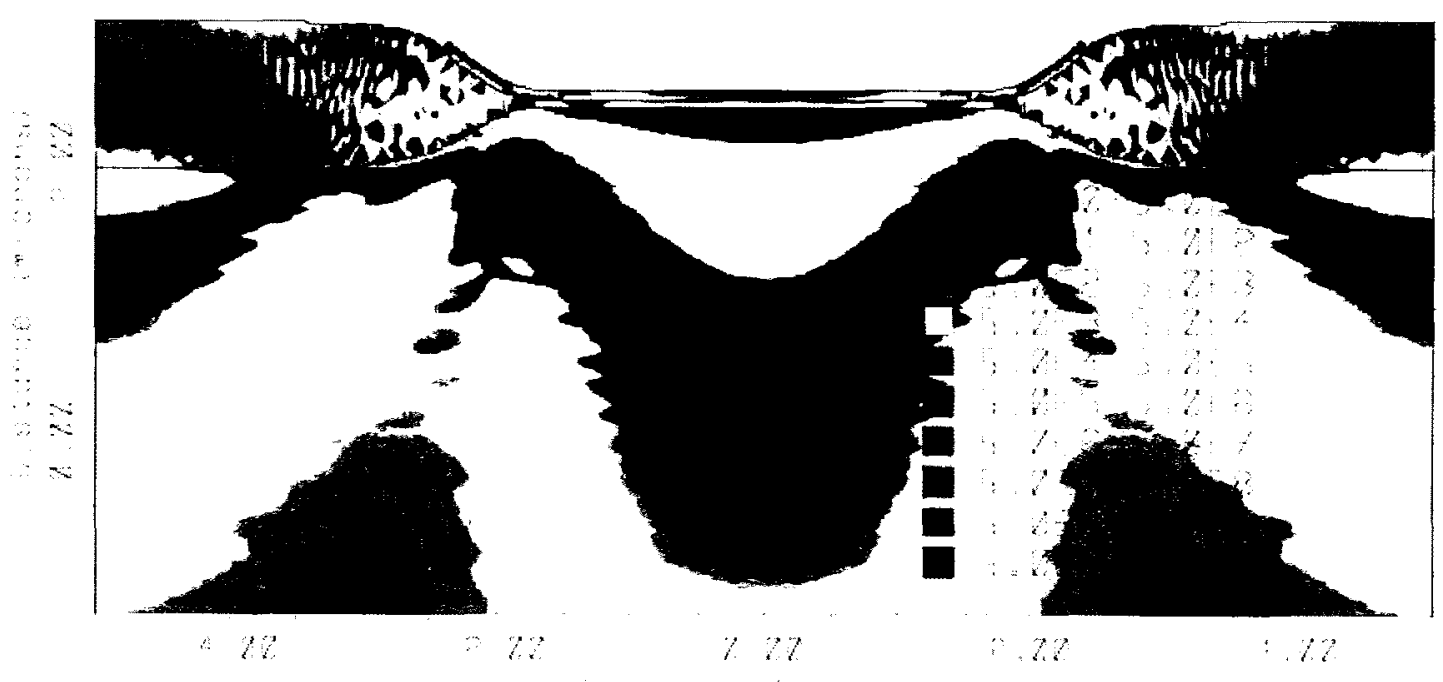

Figure 3.29: Tensile stress $\sigma_{\mathrm{xx}}\left(\mathrm{dyn} / \mathrm{cm}^{2}\right)$ due to oxidation in LOCOS waveguide structure.

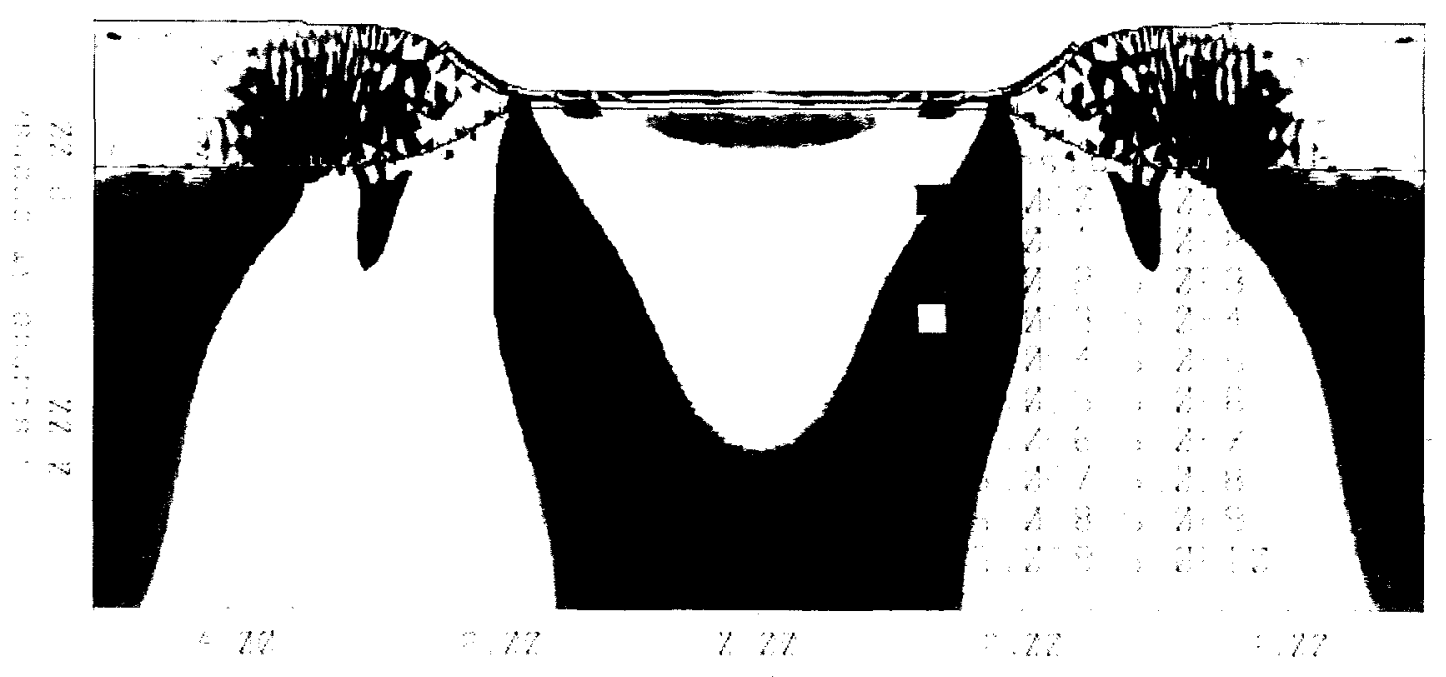

Figure 3.30: Tensile stress $\sigma_{y y}\left(\mathrm{dyn} / \mathrm{cm}^{2}\right)$ due to oxidation in LOCOS waveguide structure. 
Chapter 3 Theory

As can be seen from the above stress plots, the maximum stresses occur along the silica-silicon interface and seem to be in the hundreds of kilopascals to tens of megapascals. Stresses and the index of refraction of a material are linked via the stressoptic law [Bri05]:

$$
n_{1}-n_{2}=C\left(\sigma_{1}-\sigma_{2}\right)(3.2)
$$

where $n_{l}$ and $n_{2}$ are the principal indices of refraction and $\sigma_{1}$ and $\sigma_{2}$ are the principal stresses. The quantity that links the stresses and index of refraction is the stress-optic or photoelastic constant, $C$, whose value [Lia92] is defined by:

$$
C=\frac{n_{0}^{3}}{4}\left|\left(\pi_{11}-\pi_{12}+\pi_{44}\right)\right|
$$

where $n_{0}$ is the index of refraction of the silicon crystal when it is free from stress and in (100) silicon, $\pi_{11^{-}} \pi_{12}=-12.22 \times 10^{-14} \mathrm{~cm}^{2} / \mathrm{dyn}$ and $\pi_{44}=-6.50 \times 10^{-14} \mathrm{~cm}^{2} / \mathrm{dyn}$ [Lia92]. This yields a photoelastic coefficient of approximately $1.97 \times 10^{-12} \mathrm{~cm}^{2} /$ dyn in silicon at 1550 nm.

Stresses (particularly compressive stresses) in the oxide cladding and their effect on waveguide birefringence have been studied [Ye04], [Ye05] where the oxide cladding was deposited by plasma-enhanced chemical vapour deposition (PECVD). In these studies, compressive stresses between 100 and $320 \mathrm{MPa}$ were obtained in these oxide films to give a change in refractive index of the oxide on the order of $5 \%$, which ultimately leads to a very small change in effective index of the mode on the order of $10^{-5}$ [Ye04]. Given that the stresses observed here are 1-2 orders of magnitude less than those in that work, the change in refractive index within the oxide isolation is likely much smaller and an even smaller change in the effective index of the mode would be expected. Given the 
Chapter 3 Theory

relationship between the effective indices of the modes and the birefringence in (3.1), the oxide stress will likely have very little influence on the waveguide birefringence.

\subsubsection{Polysilicon Buffered LOCOS (PBL) Process}

The polysilicon buffered LOCOS (PBL) process involves a modified LOCOS stack with a thinner pad oxide layer with a polysilicon buffer layer over it and a thick nitride layer on the top. Here the polysilicon is used to release the stresses due to the mismatch in the thermal coefficients between the silicon nitride masking layer and the silicon substrate while reducing the lateral diffusion of oxygen that contributes to the encroachment.

In this process, the thin pad oxide was grown at $900^{\circ} \mathrm{C}$ in a dry oxygen gas ambient for 48 minutes to achieve a thickness of $10 \mathrm{~nm}$. Next, a polysilicon film $80 \mathrm{~nm}$ thick was deposited at a temperature of $550^{\circ} \mathrm{C}$. Finally, the $160 \mathrm{~nm}$ thick silicon nitride film was deposited at a temperature of $820^{\circ} \mathrm{C}$. The sample was then etched to a depth of $200 \mathrm{~nm}$ to completely remove the nitride and reach the middle of the polysilicon buffer layer over the field regions leaving a $5 \mu \mathrm{m}$ wide stack. The same three stage oxidation process at $1100^{\circ} \mathrm{C}$ as in the conventional LOCOS process was used to grow the $1 \mu \mathrm{m}$ thick field oxide in this simulation. In reality, however, most commercial CMOS processes employing this type of isolation would use a lower temperature of around $1000^{\circ} \mathrm{C}$ for the field oxidation.

The cross-sectional view of the device structure produced using this PBL process is shown in Figure 3.31 and the stresses in the structure following the field oxidation are shown in Figure 3.32 and Figure 3.34. 


$$
\text { is }
$$

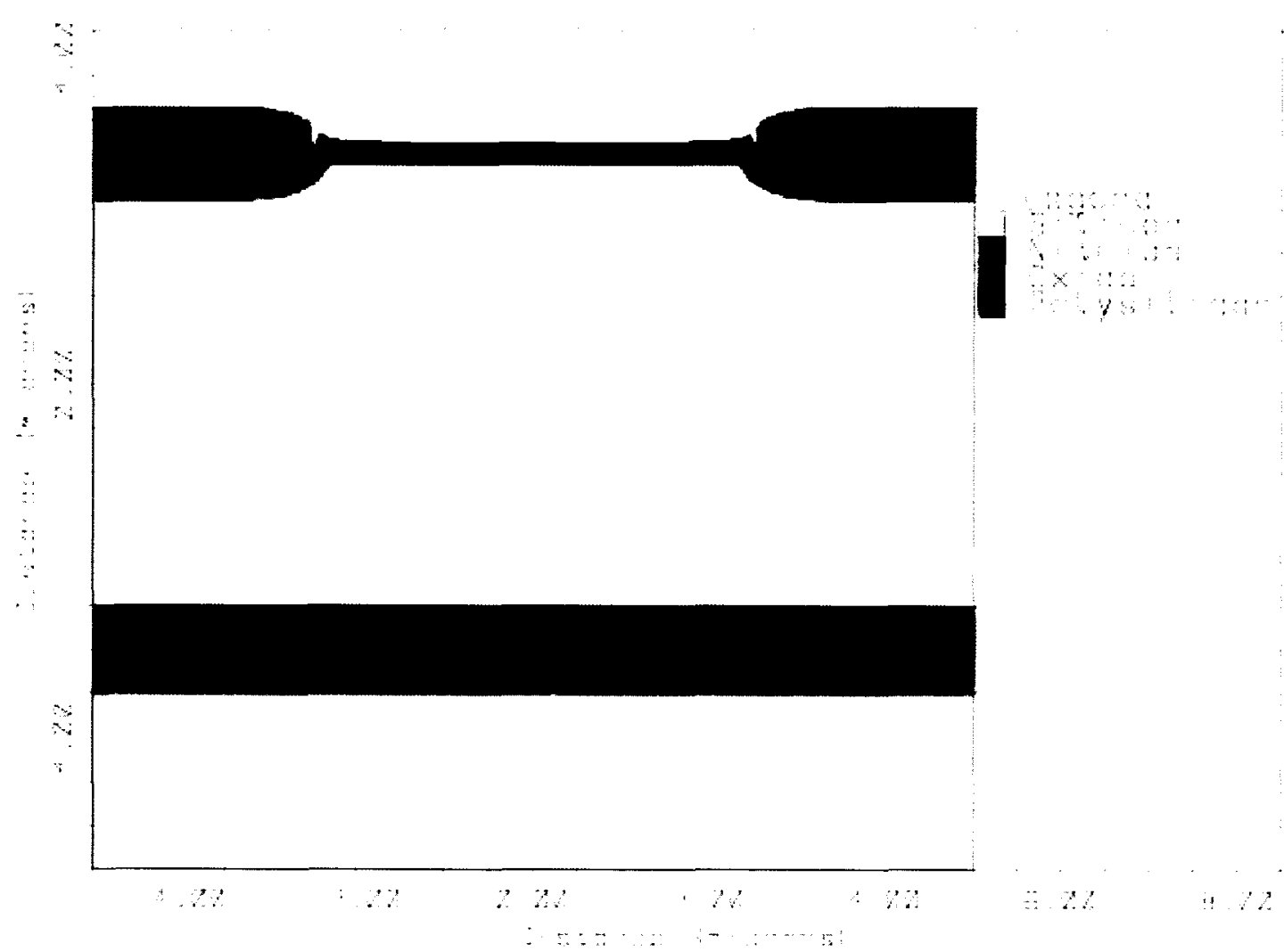

Figure 3.31: Waveguide device cross-section obtained with PBL process

As can be seen from the cross-sectional view of the waveguide device in Figure 3.31 when compared to that obtained using the conventional LOCOS method (Figure 3.26), a much smaller encroachment and steeper sidewalls have been obtained. 


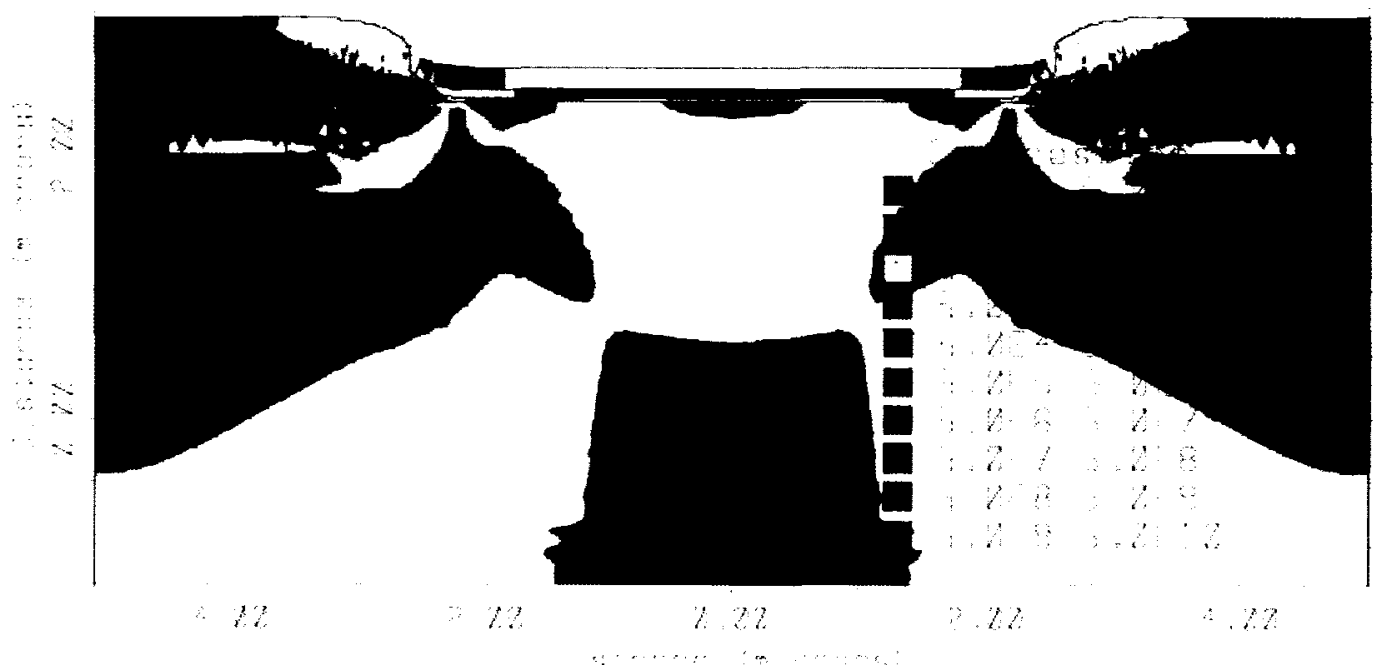

Figure 3.32: Compressive stress $\sigma_{x x}\left(d y n / \mathrm{cm}^{2}\right)$ in PBL waveguide structure

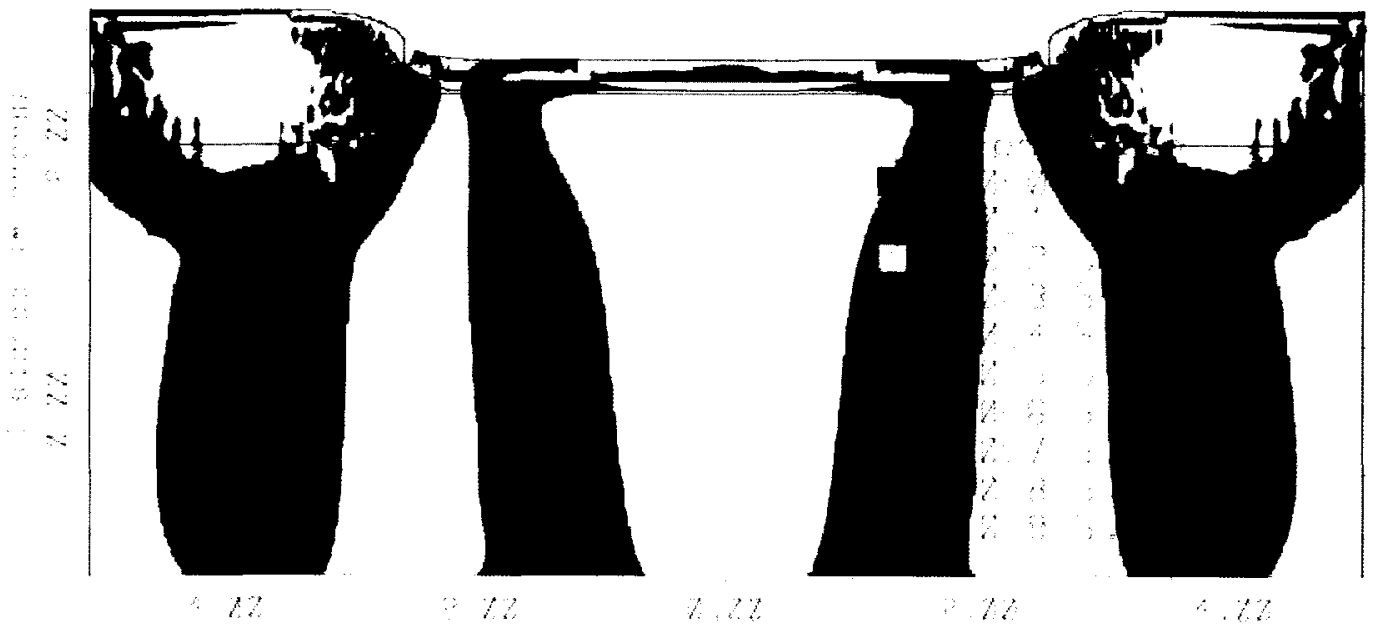

Figure 3.33: Compressive stress $\sigma_{y y}\left(\mathrm{dyn} / \mathrm{cm}^{2}\right)$ in PBL waveguide structure 


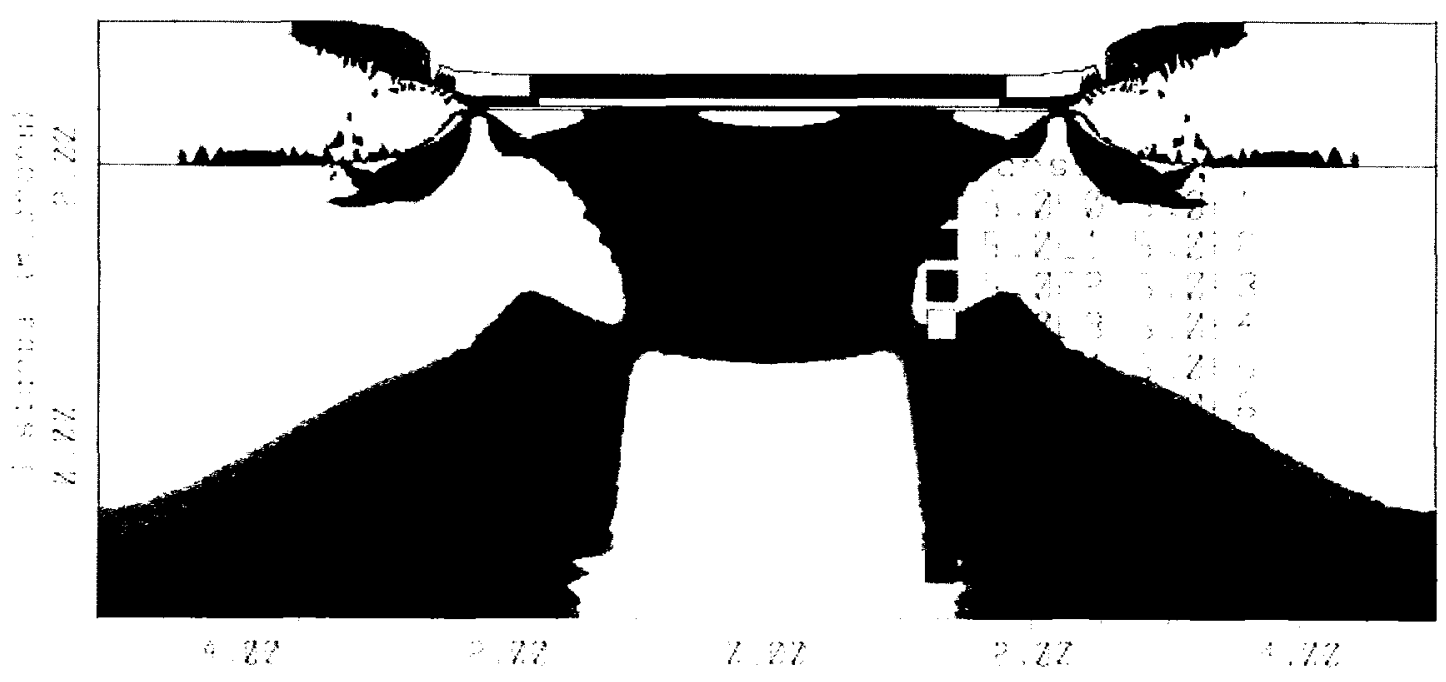

Figure 3.34: Tensile stress $\sigma_{x x}\left(\mathrm{dyn} / \mathrm{cm}^{2}\right)$ in $\mathrm{PBL}$ waveguide structure

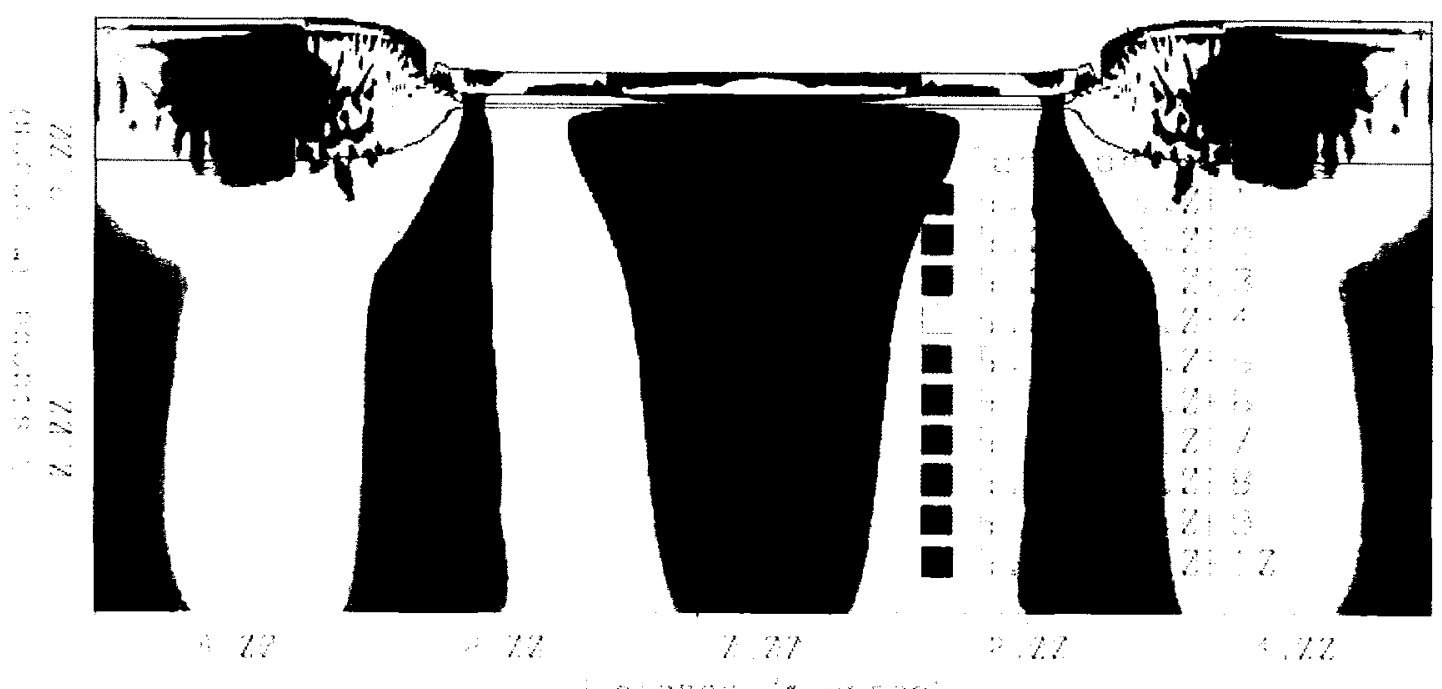

Figure 3.35: Tensile stress $\sigma_{y y}\left(\mathrm{dyn} / \mathrm{cm}^{2}\right)$ in $\mathrm{PBL}$ waveguide structure 
Chapter 3 Theory

Again, the stresses within the oxide layer are much smaller than those in [Ye04], so

little change in effective index would be expected and therefore stress-induced

birefringence effects in the waveguide will be negligible. They also seem to be roughly equal in magnitude to those in the conventional LOCOS process in the previous section.

\subsubsection{Sealed-Interface Local Oxidation (SILO)}

The sealed-interface local oxidation (SILO) process also involves a modified LOCOS stack. Here, a very thin nitride layer of $20 \mathrm{~nm}$ in thickness is deposited by CVD directly onto the silicon surface to seal it and prevent oxidation. Following that, a thin oxide layer of $30 \mathrm{~nm}$ in thickness is also deposited by CVD directly on top of the nitride. Finally a much thicker nitride layer of $150 \mathrm{~nm}$ in thickness is deposited on top of the CVD oxide to complete the LOCOS stack. The stack is then etched away over the field regions of the device and subsequently oxidized using the 3 stage oxidation method used in the Carleton nMOS process as described in section 3.4.1, though a lower temperature near $1000^{\circ} \mathrm{C}$ would likely have been used in practice.

The cross-sectional view of the device structure produced using this PBL process is shown in Figure 3.36 and the stresses in the structure following the field oxidation are shown in Figure 3.37 and Figure 3.40. 
Ha

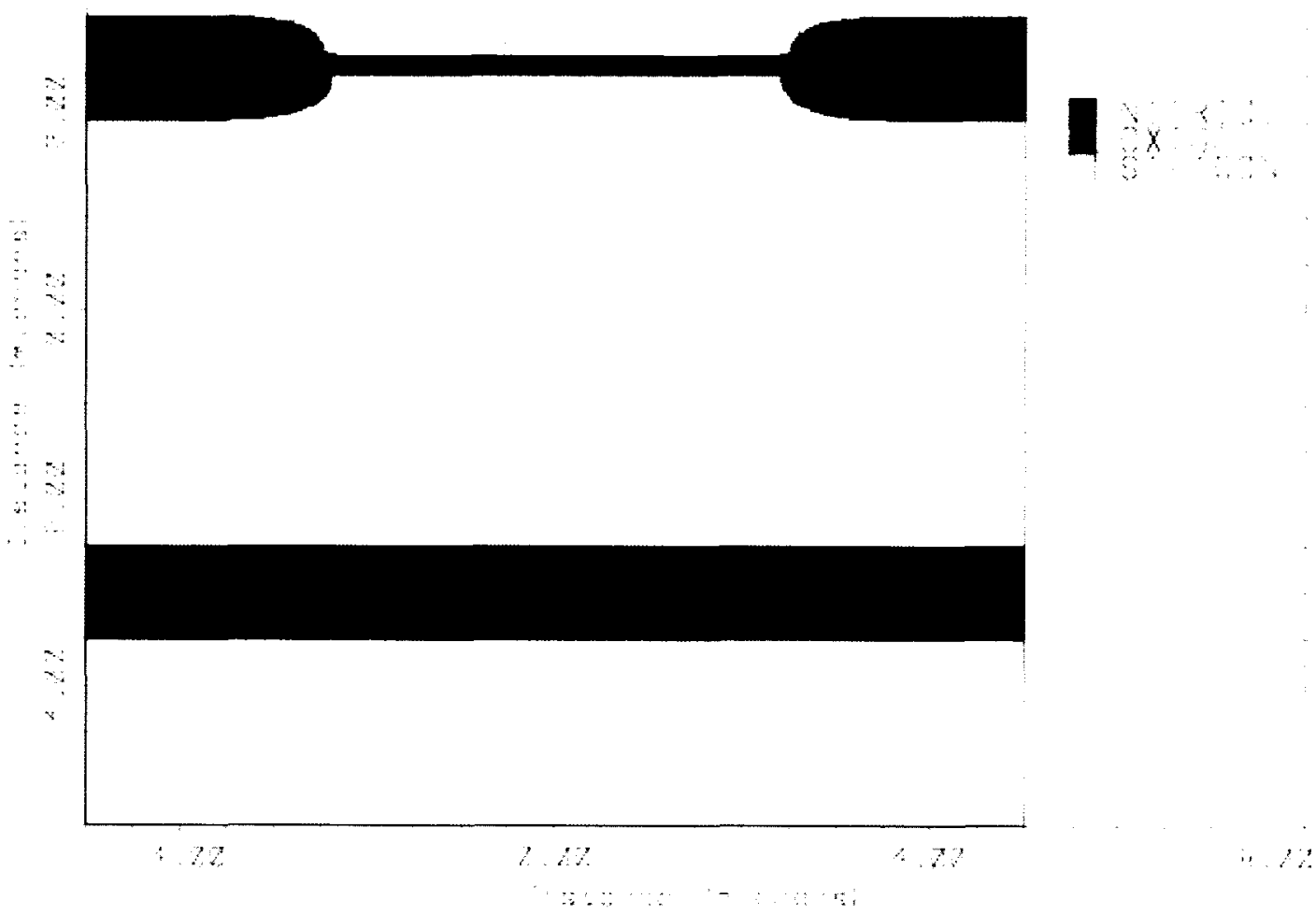

Figure 3.36: Waveguide device cross-section obtained with SILO process

Again, when the cross-sectional view of the waveguide device fabricated with this process (Figure 3.36) is compared against that obtained with the conventional LOCOS process (Figure 3.26), we can see that the encroachment due to lateral diffusion of oxygen has been reduced dramatically by the sealing of the silicon surface by the first nitride layer to produce near-vertical waveguide sidewalls. We can also see that that this is also a substantial reduction in encroachment when compared against the PBL process (Figure 3.31). 


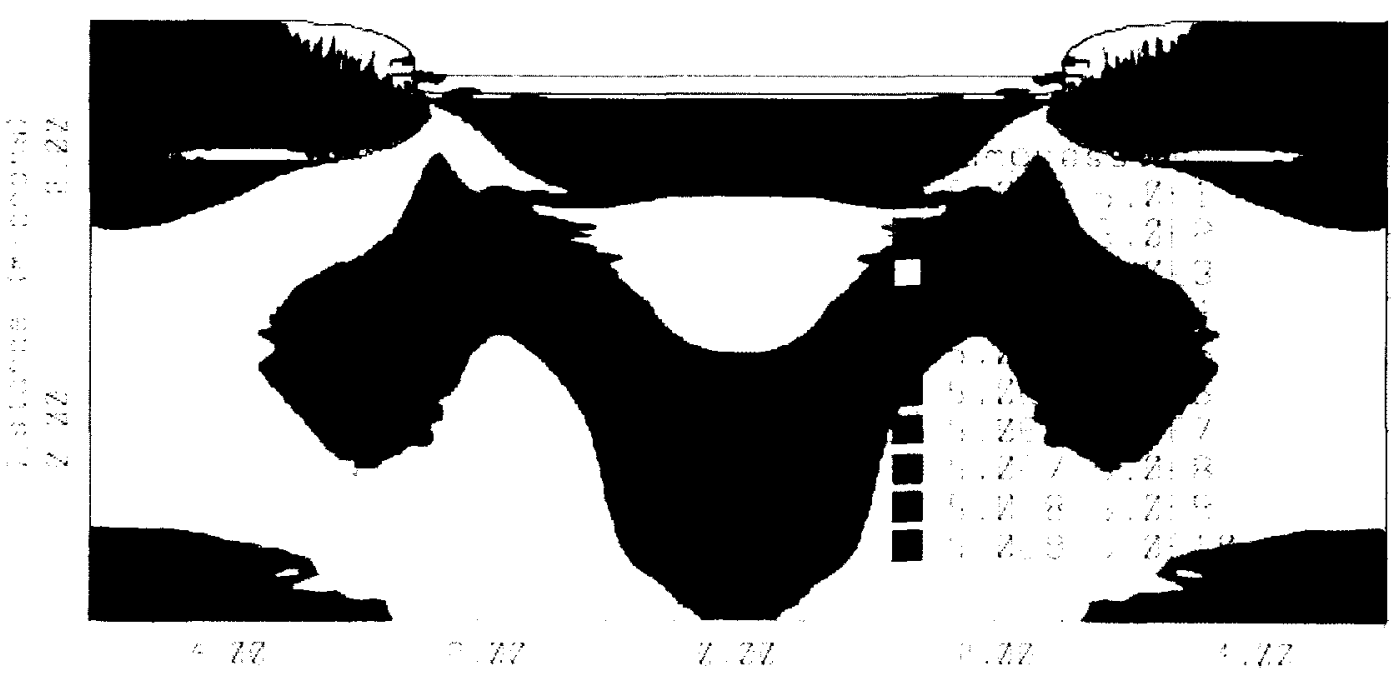

Figure 3.37: Compressive stress $\sigma_{x x}\left(d_{y n} / \mathrm{cm}^{2}\right)$ in SILO waveguide structure

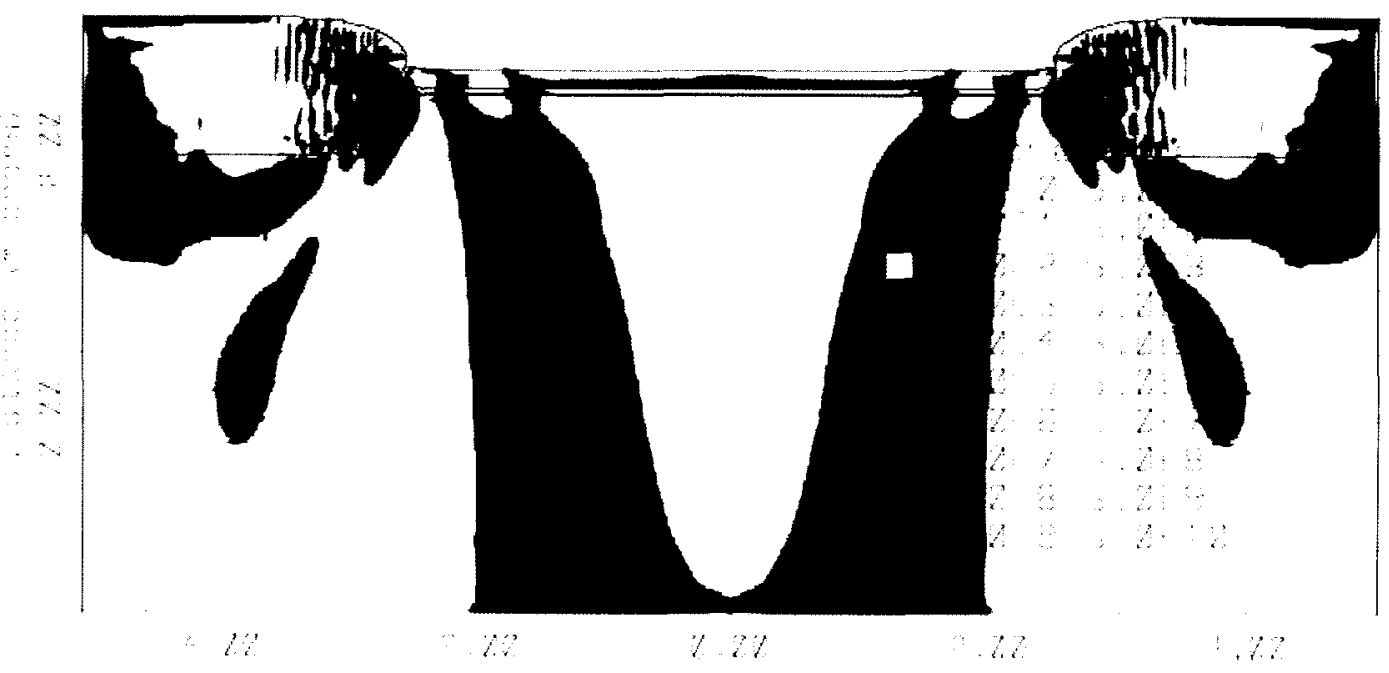

Figure 3.38: Compressive stress $\sigma_{y y}\left(\mathrm{dyn} / \mathrm{cm}^{2}\right)$ in SILO waveguide structure 


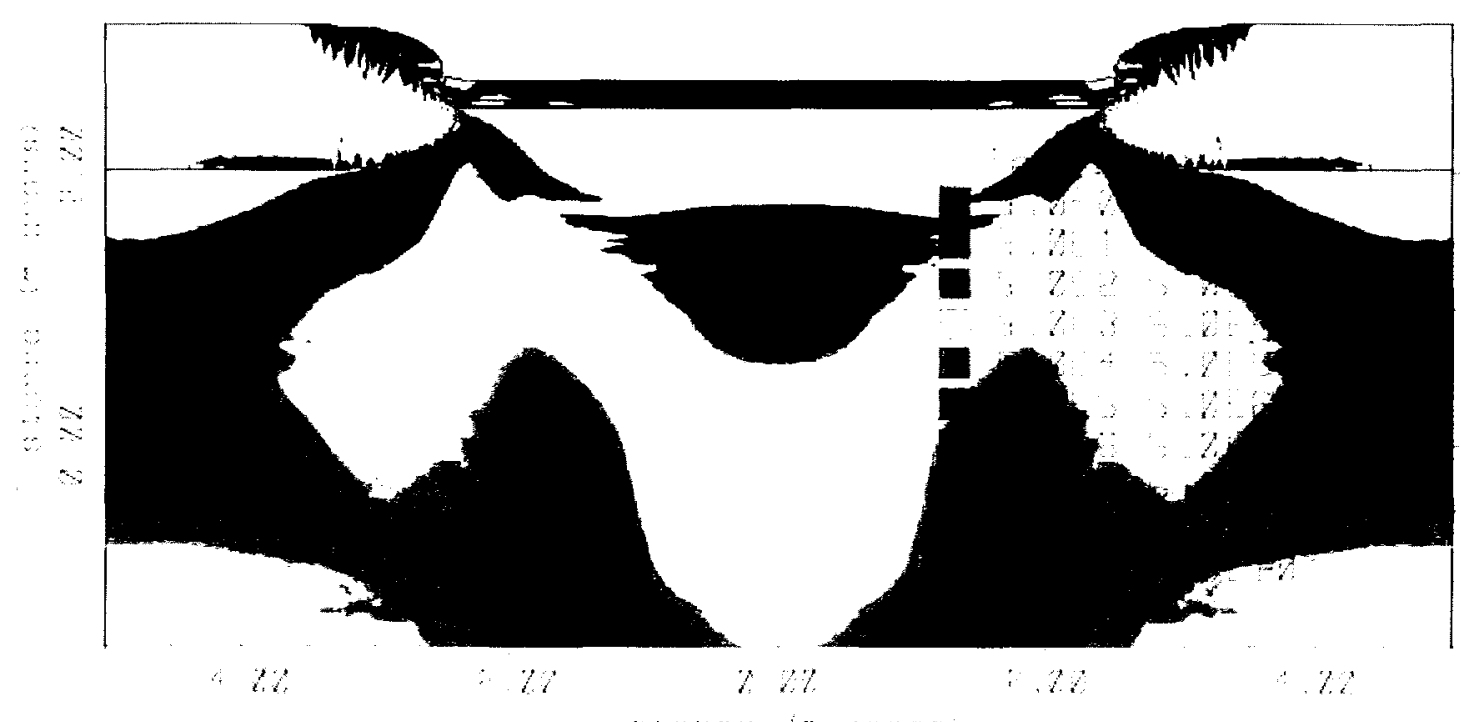

Figure 3.39: Tensile stress $\sigma_{\mathrm{xx}}\left(\mathrm{dyn} / \mathrm{cm}^{2}\right)$ in SILO waveguide structure

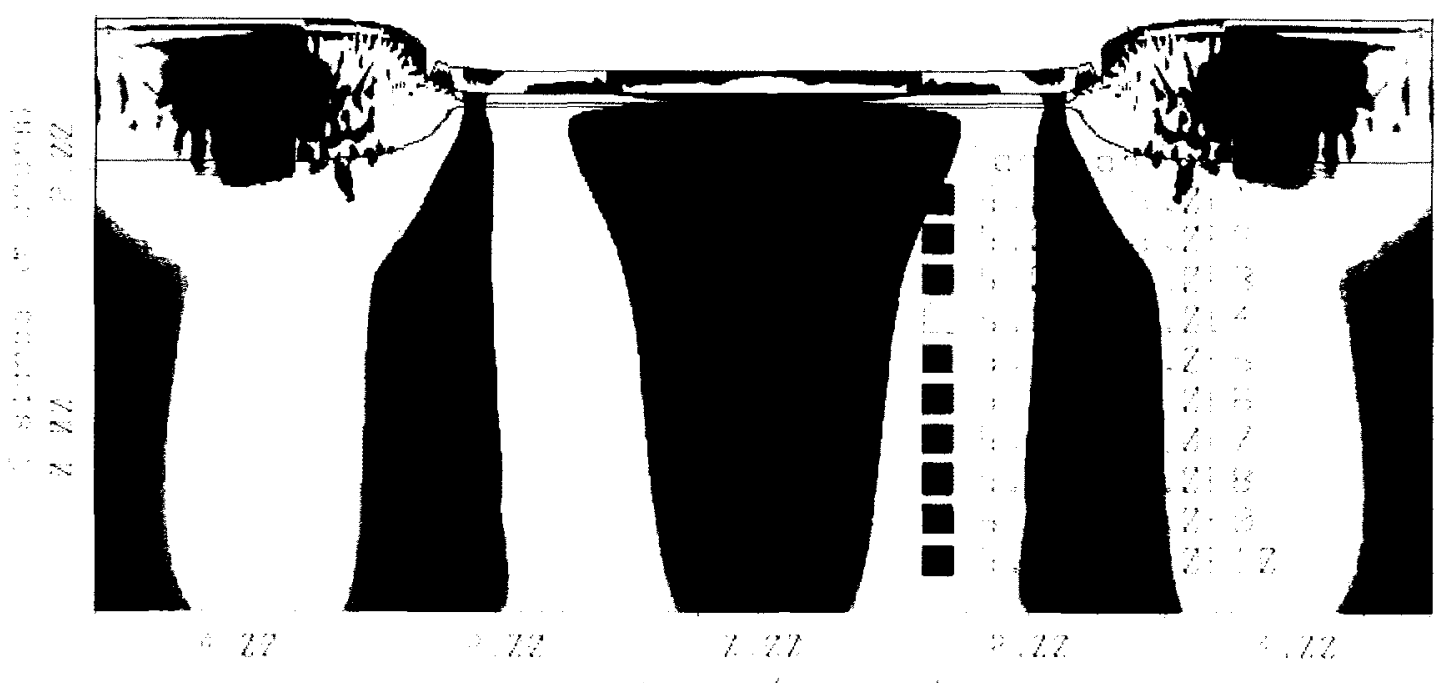

Figure 3.40: Tensile stress $\sigma_{y y}\left(\mathrm{dyn} / \mathrm{cm}^{2}\right)$ in SILO waveguide structure 
Chapter 3 Theory

As with the conventional LOCOS and the PBL processes presented in the two previous sections, we see that we have stresses of the same range of hundreds of kilopascals to tens of megapascals range. As these are a few orders of magnitude less than those in [Ye04], we expect very little change in the waveguide birefringence due to these stresses. 


\section{Chapter 4 Fabrication}

In this chapter, the detailed process flow that was used in fabricating the LOCOS waveguide devices in the Carleton University Microfabrication Facility will be discussed. The section will begin by describing the mask and SOI material that was used in fabricating the waveguides, as well as the individual steps such as the pad oxide growth, nitride deposition, photolithography, plasma etching, and field oxidation. The final structure obtained in this process and the end-facet preparation required before buttcoupling to a tapered fibre for optical testing are also discussed.

\subsection{Waveguide Mask}

The LOCOS waveguides in this project were formed with a chrome mask designed by Phil Waldron at McMaster University in a previous project. This mask contains a selection of straight waveguide patterns as well as devices such as balanced and unbalanced Mach-Zehnder Interferometers (MZIs) and Y-splitters. The layout of this mask is shown in Figure 4.1. 
Chapter 4 Fabrication

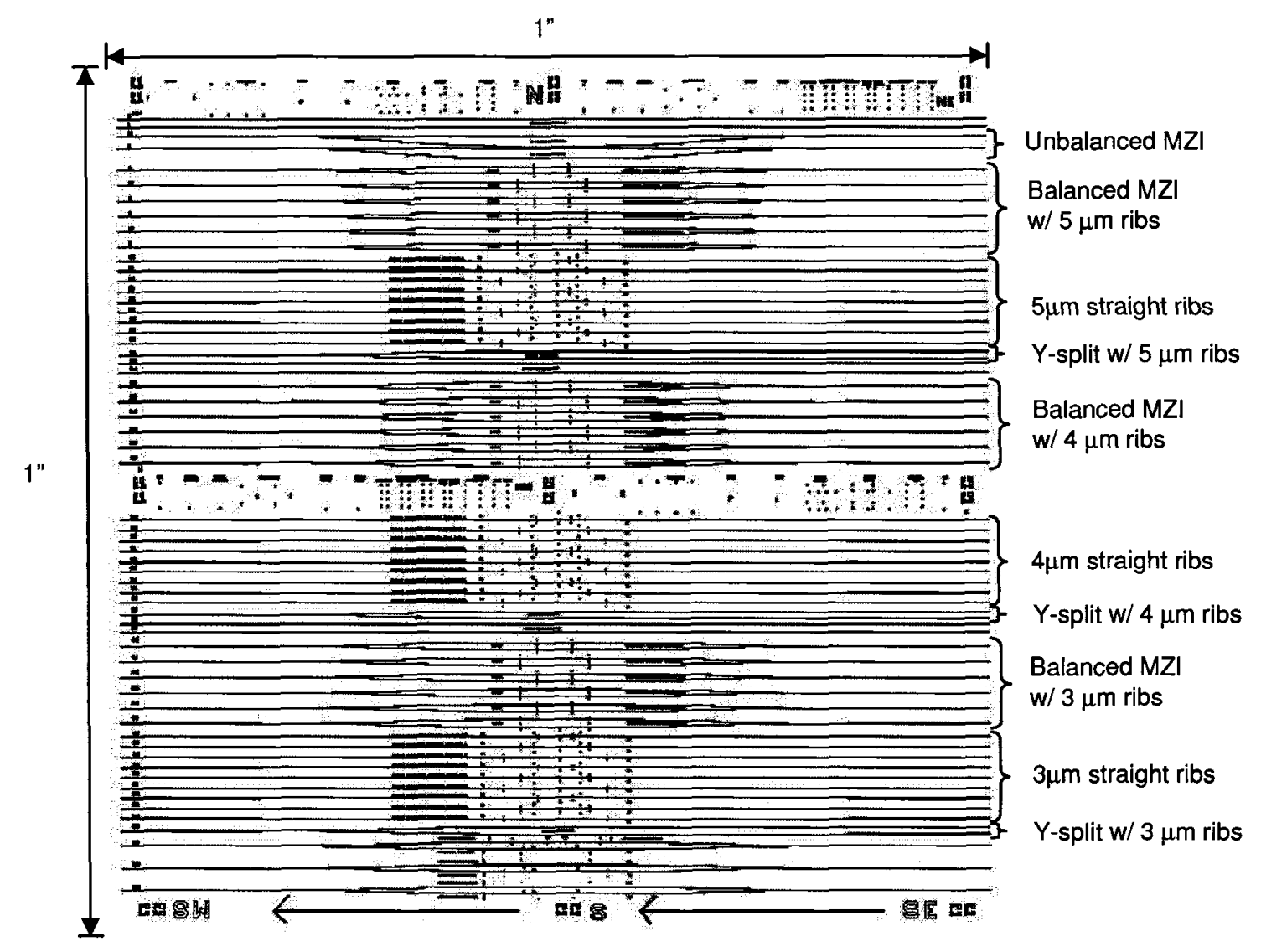

Figure 4.1: Rib waveguide mask layout by Phil Waldron (McMaster University)

As mentioned, a selection of straight waveguides, balanced MZIs, and Y-splitters in 3

$\mu \mathrm{m}, 4 \mu \mathrm{m}$ and $5 \mu \mathrm{m}$ widths are included on this mask and it is these patterns that will be transferred by photolithography to form the LOCOS stacks prior to the field oxidation stages used to form the slab regions of the optical rib waveguide. Although this mask was originally designed for masking RIE etched waveguide ribs, this mask does possess the contrast needed to pattern the LOCOS stack in this project.

\subsection{SOI material overview}

In this project, two different SOI materials with different silicon film thicknesses and buried oxide thicknesses were used when fabricating the waveguides. The first material was $8 \Omega \mathrm{cm}$ p-type bond-and-etchback SOI from SEH with a $2.5 \mu \mathrm{m}$ silicon film 
Chapter 4 Fabrication

thickness and a $1 \mu \mathrm{m}$ buried oxide. The second SOI material that was used is approximately $7 \Omega \mathrm{cm}$ p-type Smart-cut SOI from SOITEC with a $3.4 \mu \mathrm{m}$ silicon film on a $0.4 \mu \mathrm{m}$ buried oxide layer. All wafers had a (100) crystal orientation.

The bond-and-etchback (BESOI) [Ja198] process is one in which a silicon wafer is thermally oxidized and then bonded to another silicon wafer and subjected to a heat treatment. The first wafer surface is then polished to the required desired silicon film thickness by either purely mechanical means or by a combination of chemical and mechanical means. However, the polishing methods result in a very poor uniformity in the thickness of the silicon layer [Ja198] on the order of $\pm 500 \mathrm{~nm}$ [Kya05] which leads to non-uniformity in the $h / H$ ratio and hence to the possibility of localized multimode behaviour.

The smart-cut process is one in which a silicon wafer is oxidized and implanted with hydrogen ions. The oxidized wafer is then bonded to another wafer and subjected to a heat treatment. The heat causes the wafer to blister where the hydrogen ions were implanted to split the wafer. Residual microscopic surface defects are removed by a chem-mechanical polishing process. The implantation process results in a much better thickness uniformity [Jal98] of $\pm 5-20 \mathrm{~nm}$ [Kya05], [Soi05] with an oxide thickness comparable to that in the BESOI process [Kya05], [Jal98].

One 4-inch wafer of each SOI type was cut into $1 \frac{1 / 4}{4}$-inch square pieces and two of the pieces from each wafer were used for the remainder of the fabrication process. Each piece of SOI was also scribed on the backside with a number to identify it throughout the subsequent processing stages. The two pieces cut from the SEH wafers were labelled L1 and L2, while the pieces cut from the SOITEC wafers were labelled L4 and L5. 


\subsection{Process flow}

\subsubsection{Pad Oxide Growth}

To begin the LOCOS process flow, a thin pad oxide layer must be grown over the entire silicon surface to relieve the stresses from the mismatch in the thermal expansion between the silicon nitride masking layer and the silicon substrate. A $50 \mathrm{~nm}$ thick layer of thermal oxide was grown at $1100^{\circ} \mathrm{C}$. To confirm that the desired oxide thickness had been achieved, blank $2 \Omega \mathrm{cm}$ p-type silicon wafers were also oxidized along with the SOI. Following the oxidation, the thermal oxide layer thickness on one of these wafers was measured with the Nanospec interferometer.

\subsubsection{Nitride Deposition}

In addition to the pad oxide, the other main component of the LOCOS stack is the silicon nitride layer used to mask the field oxidation. This silicon nitride layer is deposited on top of the thermal pad oxide layer using a low pressure chemical vapour deposition process in which ammonia and dichlorosilane gases react according to:

$3 \mathrm{SiH}_{2} \mathrm{Cl}_{2}+4 \mathrm{NH}_{4} \rightarrow \mathrm{Si}_{3} \mathrm{~N}_{4}+6 \mathrm{HCl}+8 \mathrm{H}_{2}$

and the silicon nitride produced accumulates on the wafer surface [Plu00]. This reaction (4.1) is carried out inside a furnace tube heated to a temperature of $820^{\circ} \mathrm{C}$ and, at this temperature, 11 minutes in an ambient containing these gases is sufficient to deposit the required $80 \mathrm{~nm}$ silicon nitride film.

Again, to confirm the thickness of the deposited silicon nitride layer, $2 \Omega \mathrm{cm}$ p-type $2 "$ silicon wafers are included with the SOI and the thickness of the silicon nitride layer is measured with the Nanospec. 
Chapter 4 Fabrication

\subsubsection{Photolithography}

To define the LOCOS stack regions used to mask the waveguide ribs during field oxidation, photolithography is used to transfer the mask pattern to the surface of the silicon nitride and silicon dioxide pad layers on the wafers.

Before the photoresist can be spun onto the SOI surface, the pieces were subjected to a dehydration baking to help drive away any accumulated moisture that may prevent the photoresist from adhering to the wafer surface. Each one of the SOI pieces was attached to a 2 " silicon wafer using a single drop of photoresist applied to the centre of the wafer. The back of the SOI piece was then pressed on top of the photoresist drop and the wafer was baked at $115^{\circ} \mathrm{C}$ for 3 minutes to make the resist harden to act like a glue to hold the SOI pieces on the silicon wafer surface. Next, a layer of hexamethyldisilane (HMDS) was spun on to the SOI surface and baked. This layer of HMDS is used to promote the adhesion of the photoresist to the SOI surface.

The Shipley S1811 positive photoresist was then spun onto the SOI surface and given a pre-exposure bake. The mask was aligned such that the ribs ran parallel to one of the cleaved edges of the SOI pieces and was exposed for 15 seconds in the hard contact mode of the Karl Suss MA6 mask aligner.

The pattern was developed in MF321 commercial developer solution to remove the exposed photoresist. The wafers were subsequently rinsed in de-ionized water and a subjected to post-exposure bake. The SOI pieces were removed from the 2" backing wafers and the photoresist residue on the back of the SOI pieces was removed by swabbing in acetone. Any exposed photoresist residue that remained was removed by an 
Chapter 4 Fabrication

oxygen plasma descum at 0.3 Torr and $100 \mathrm{~W}$ power in the Technics Planar Etch II planar plasma etcher.

\subsubsection{Reactive Ion Etching}

Once the LOCOS stack pattern was transferred to the SOI surface by the

photolithography process, the exposed silicon nitride was removed over the field regions by plasma etching. A Technics Planar Etch II planar plasma etcher with source gases of Freon and 5\% oxygen was used for this purpose In this case a reaction between the Freon gas $\left(\mathrm{CF}_{4}\right)$ and the free electrons in the plasma causes the dissociation reaction [Plu00]:

$\mathrm{e}^{-}+\mathrm{CF}_{4} \rightarrow \mathrm{CF}_{3}+\mathrm{F}+\mathrm{e}^{-}(4.2)$

which produces two free radicals or electrically neutral chemical species that have unpaired electrons, $\mathrm{F}$ and $\mathrm{CF}_{3}$. Due to this incomplete bonding structure, the fluorine atoms can easily bond to the silicon in the silicon nitride to react to form $\mathrm{SiF}_{4}$ gas according to [Plu00]:

$4 \mathrm{~F}+\mathrm{Si} \rightarrow \mathrm{SiF}_{4}(4.3)$

However, it is important to note that the reverse of the reaction in (4.2) can also occur which has the undesirable effect of reducing the amount of fluorine available for the desired reaction in (4.3). The addition of the $5 \%$ oxygen reacts with some of the $\mathrm{CF}_{3}$ produced by (4.2) so that there is less of it available to recombine in the reverse of (4.2) [Plu00]. This ensures that there will always be some free fluorine atoms available to participate in reaction (4.3) and etch away the silicon nitride layer. 
Chapter 4 Fabrication

\subsubsection{Field oxidation}

The final stage of the LOCOS fabrication process is the field oxidation step in which a

$1 \mu \mathrm{m}$ field oxide was grown by thermal oxidation in the field regions to produce the waveguide slab regions. Before this final stage the pad oxide layer was removed on SOI samples L2 and L5 by a wet chemical etch in buffered HF and left in place on samples L1 and L4 to observe the difference in the extent of the bird's beak produced.

The samples were processed at the same $1100^{\circ} \mathrm{C}$ temperature in the same furnace as for the pad oxidation step. However, in this case, the $1 \mu \mathrm{m}$ oxide was grown in a sequence of three steps. First, the wafer was subjected to a dry $\mathrm{O}_{2}$ gas, as was done for the pad oxidation, for a duration of 10 minutes. Then, a wet oxygen or steam ambient was used to grow most of the $1 \mu \mathrm{m}$ thickness over a duration of 150 minutes. Finally, a second dry oxidation step with the addition of $2.5 \% \mathrm{HCl}$ to reduce stacking faults [Hat78] was done to reach the final thickness in the last 30 minutes of the process. As with the pad oxide, a thermal annealing step was also carried out in a nitrogen ambient at $1100^{\circ} \mathrm{C}$ for 40 minutes. 
Chapter 4 Fabrication

\subsection{Fabricated Waveguide Structure}

The LOCOS waveguide structure fabricated according to the process described in the previous section is shown in the Scanning Electron Microscope (SEM) image in Figure 4.2 .

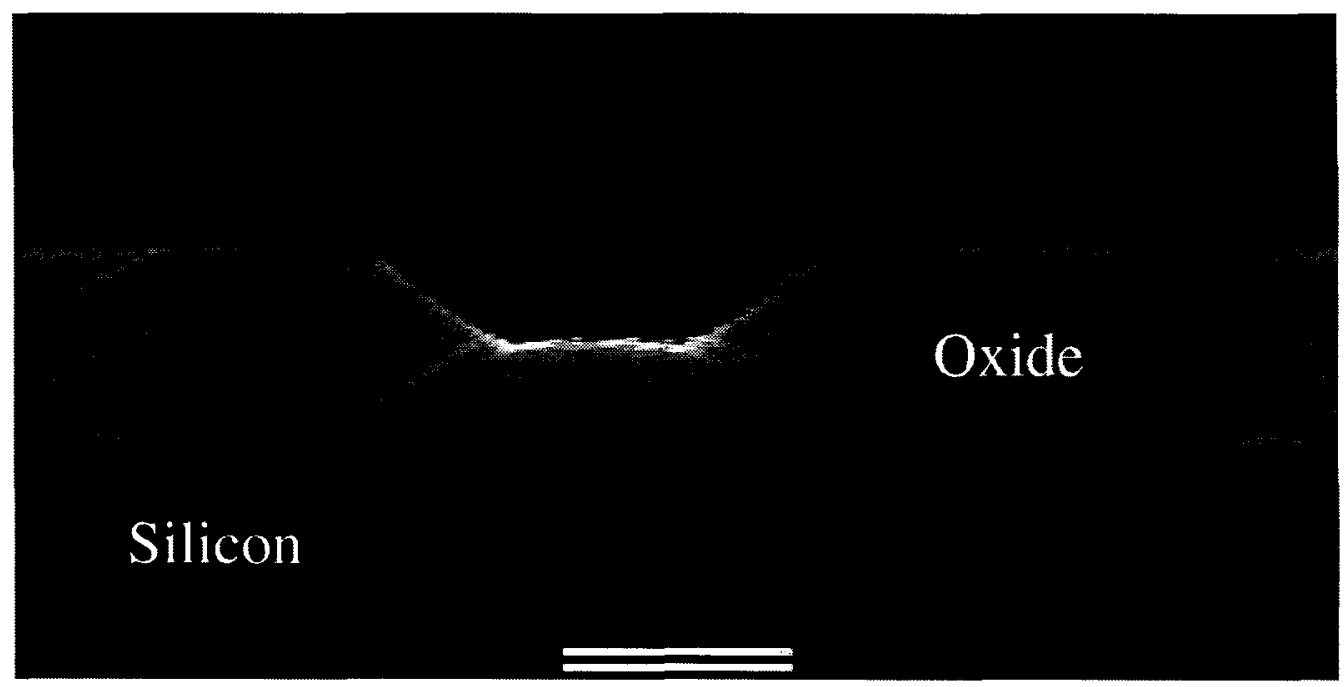

Figure 4.2: SEM cross-section view of LOCOS waveguide structure after LOCOS stack removal at $15000 \mathrm{X}$ magnification. The white bars show a $1 \mu \mathrm{m}$ length.

As predicted by the simulation in section 3.4.1, we see that the bird's beak extension of about $0.5 \mu \mathrm{m}$ in length is present on the fabricated sample which gives us the sloped waveguide sidewalls. Our final rib width appears to be about $1 \mu \mathrm{m}$ for this rib with a 3 $\mu \mathrm{m}$ drawn rib width. We can also see that we have our semi-recessed oxide with a $1 \mu \mathrm{m}$ thickness covering our slab region to obtain an effective etch depth of around $0.5 \mu \mathrm{m}$ for the rib. 
Chapter 4 Fabrication

\subsection{End Facet Preparation for Testing}

In order to test the waveguide structure it is necessary to couple light from a tapered fibre into the waveguide rib. The easiest way of accomplishing this is to butt-couple the fibre to the waveguide facet as shown in Figure 4.3.

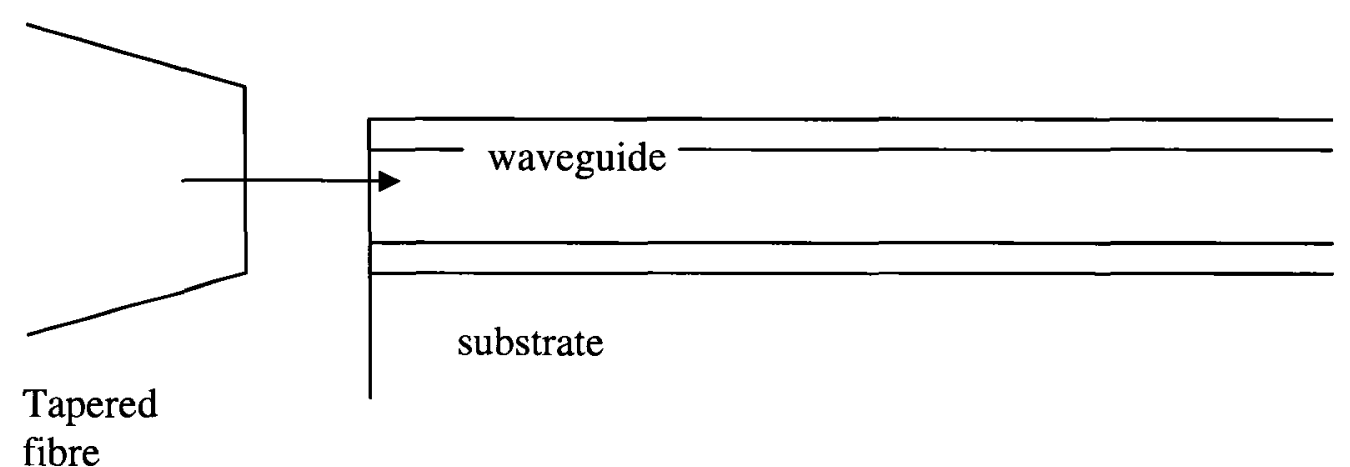

Figure 4.3: Side view of tapered optical fibre butt-coupled to waveguide end facet

However, to ensure a minimal insertion loss when coupling light into the fibre in this manner, care must be taken in obtaining a very smooth end facet on the waveguide normal to the direction of light propagation within the waveguide. A similar smooth end facet is also required at the output in order to avoid loss when coupling to the microscope objective lens and infrared camera. Any imperfections in either facet of the waveguide can lead to scattering losses. Although many methods of obtaining the required optical quality end facets exist, the simplest method is to just thin and cleave the SOI sample.

Since our SOI sample consists of layers of silicon with crystal orientation (100), the sample will naturally break or cleave easily along the (110) crystal planes which run parallel and perpendicular to the sample edges. When aligning the waveguide mask during photolithography, care was taken to align the ribs such that the required end facets 
Chapter 4 Fabrication

are parallel to these (110) crystal planes such that they can be easily obtained by cleaving. To obtain an optical quality cleaved facet, it was necessary to thin the samples from their initial $550-600 \mu \mathrm{m}$ thickness down to a thickness of $200-250 \mu \mathrm{m}$. This thinning process was completed in the Centre for Emerging Device Technologies (CEDT) Integrated Optics Research lab and the Materials Science \& Engineering Research lab at McMaster University.

The initial thickness of the samples were measured using a Scherr Tumico Dial Indicator, which consists of a spring loaded tip connected to the needle on the dial that registers the thickness based on the extent of the compression of the spring, and the initial thickness was recorded. Following this, they were attached to the surface of a cylindrical brass block with a wax-like commercial adhesive product known as Crystalbond in the CEDT Integrated Optics Research lab. The block was heated to approximately $120^{\circ} \mathrm{C}$, which is the melting point of Crystalbond, on a hotplate and a small amount of Crystalbond was applied to the surface of the block. The SOI sample was pressed facedown into the liquid Crystalbond and some gentle pressure was applied to the SOI with tweezers to remove any air bubbles that may have accumulated under the sample and would lead to an uneven surface thickness. The block was then removed from the hotplate and the pressure continued to be applied to the SOI to prevent the accumulation of any air bubbles in the Crystalbond until it had solidified. The block was allowed to cool further until it reached room temperature before proceeding.

The backside of the SOI sample was ground down to the required thickness on a grinding wheel fitted with waterproof silicon carbide paper in the Materials Science and Engineering Research lab. Gentle pressure was applied on the top of the polishing block 
Chapter 4 Fabrication

and the block was continuously rotated to maintain a uniform polished SOI surface.

Periodically, the block was removed from the grinding wheel and the thickness was measured using the Scherr Tumico Dial Indicator. A coarse grit of silicon carbide paper was used to quickly reach the approximate desired thickness and a finer grit was used near the end of this process to remove any large scratches in the SOI surface before cleaving.

Once the desired SOI thickness of $200-250 \mu \mathrm{m}$ was reached, the block was heated again on a hotplate until the Crystalbond melted and the SOI sample was carefully lifted off of the surface of the block with tweezers. The Crystalbond residue on the SOI surface was removed by rinsing in acetone, methanol, and finally in de-ionized water.

The samples that were tested at McMaster University's CEDT Integrated Optics Research lab had small scribe marks made using a Karl Suss HR 100 Manual Scriber in the CEDT Electrical Characterization lab. To cleave the samples, a probe tip was aligned to the scribe mark and lowered to apply pressure at a single point on the scribe mark. The sample cleaved along the crystal plane at this location. This procedure of scribing followed by cleaving was used to obtain the input and output facets of the waveguides.

The remaining samples that were tested at the National Research Council Canada had small scribe marks made in the silicon surface with a diamond scribing pen and the samples were cleaved by flipping the sample over and placing the pointed surface of an Allen key directly under the scribe mark. Pressure was then applied to the back of the samples to obtain the desired cleave along the crystal planes. As with the other samples, this process was followed to obtain the optical facets at the input and output of the waveguides. 


\section{Chapter 5 Results and Discussion}

In this chapter, the waveguide testing work completed at McMaster University in Hamilton, Ontario as well as at the Institute for Microstructural Sciences of the National Research Council Canada in Ottawa, Ontario will be discussed in detail. In particular, the cutback measurements of the straight waveguide sections to determine the propagation losses within the LOCOS waveguides, waveguide mode profiles, the performance of the unbalanced Mach-Zehnder and Y-split devices will be covered.

\subsection{Experiment Set-up and Waveguide Alignment Procedure}

As mentioned in section 4.5, for optical testing, light from a tapered optical fibre is coupled into the SOI waveguide through the cleaved end facet of the device. However, the actual experimental set-up used is a bit more complex than this.

The experimental set-up used for the measurements at the Institute for Microstructural Sciences (IMS) of the National Research Council Canada (NRC) is shown in below. A similar experimental setup was also used for the optical testing done at the Department of Engineering Physics at McMaster University with a few minor changes that will be described. 


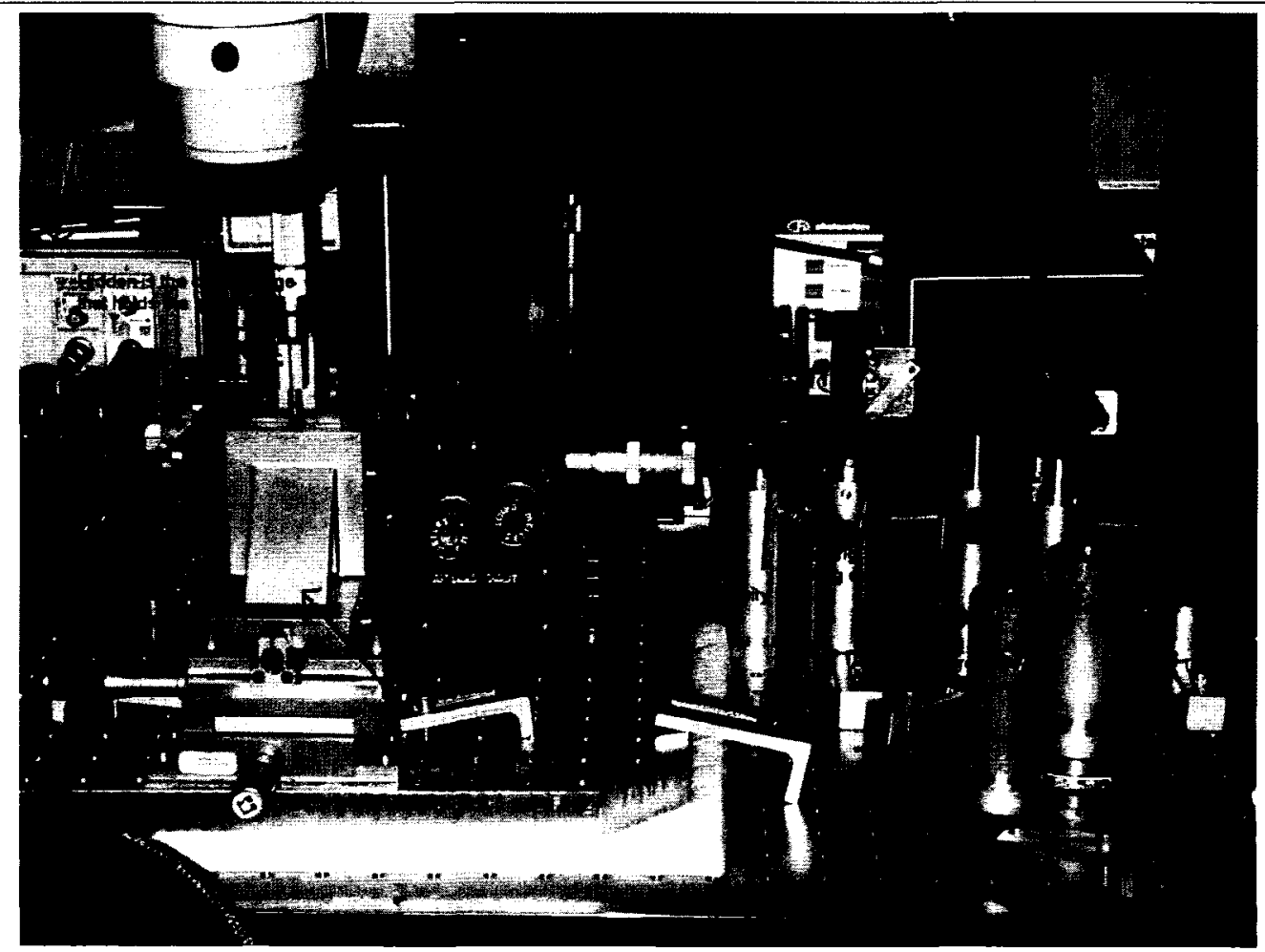

Figure 5.1: Experimental setup used for optical testing at National Research Council Canada

As can be seen from Figure 5.1, light from either a broadband or continuous wave laser source in the $1550 \mathrm{~nm}$ telecommunications band can be butt coupled into the tapered optical fibre. The waveguide sample is placed on the stage on a vibration-free optical table and its position is controlled by several micrometers (not visible in figure) similar to those on the extra stage. The tip of the optical fibre, which has been threaded through a guide, can also be positioned using micrometers which are visible on the left hand side of the figure. Similarly, the position of the microscope objective lens used to focus the light at the output face of the waveguide can be adjusted using the three micrometers which are visible on the right hand side of the stage in Figure 5.1. The light is then propagated through free space through a neutral density filter and reflected off of a mirror to reach the IR camera lens to view the optical mode or through an aperture to the photodetector to measure the output power. A similar optical testing setup exists at McMaster with the 
Chapter 5 Results and Discussion

exception that the position of the optical fibre is controlled by a piezoelectric stage and that additional mirrors were used to provide an image of the optical facet as seen through the input fibre to assist in fibre alignment.

Alignment of the fibre to the optical waveguide was performed by first adjusting the $\mathrm{x}$ y positioning of the sample stage and then by adjusting the horizontal position of the tapered fibre with the coarse settings of the micrometer such that the pointed tip lay in approximately the centre of the waveguide rib when viewed under the microscope. The vertical position of the fibre was also adjusted with the coarse adjustment of the micrometer knob such that the tip appeared to be in the same plane as the waveguide rib by observation under the microscope. The fibre tip was then brought into proximity of the input facet of the waveguide with the coarse adjustment of the micrometer while carefully observing its position under the microscope. Next, the horizontal position of the microscope objective was carefully adjusted such that the centre of the lens was aligned with the centre of the waveguide rib and its proximity to the waveguide output facet was adjusted until the spacing between the facet and the lens was equal to the focal length with the coarse alignment of the micrometers. Finally, fine adjustments to the positioning of the fibre and the microscope objective were made using the micrometer while observing the mode profile with the IR camera and then by maximizing the optical power reading obtained from the photodetector. This process was followed for each waveguide or optical device being tested. 
Chapter 5 Results and Discussion

\subsection{Waveguide Mode Profiles (NRC and McMaster)}

To find out about the ability of the waveguides to support guided modes, light was

coupled into the waveguides and the image of the waveguide mode visible at the output facet of the waveguide was focussed through the IR camera lens and displayed on a television monitor. In this case, the $3 \mu \mathrm{m}$ wide LOCOS waveguides were illuminated with TE and TM light and the output images obtained for each are shown in Figure 5.2 and Figure 5.3.

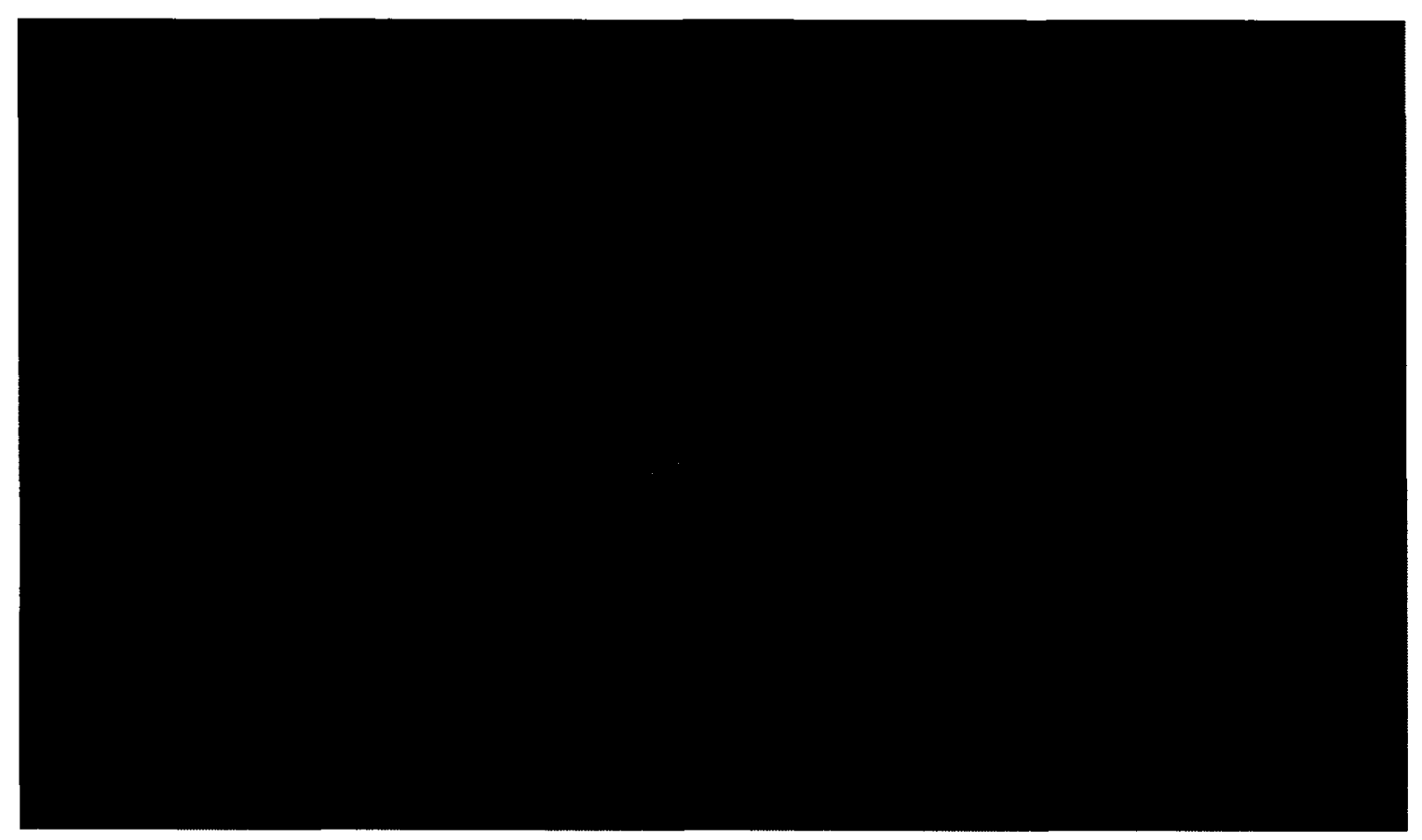

Figure 5.2: Fundamental TE mode observed for $3 \mu \mathrm{m}$ wide LOCOS isolated rib waveguide tapered to a final width of $15 \mu \mathrm{m}$. 
Chapter 5 Results and Discussion

Figure 5.3: Fundamental TM mode observed in $3 \mu \mathrm{m} \mathrm{LOCOS}$ isolated waveguide tapered to a final width of $15 \mu \mathrm{m}$.

In addition, at McMaster University, a more detailed mode profile showing the distribution of the light intensity within the mode was obtained for the $3 \mu \mathrm{m}$ waveguide. The waveguide output was fed to a CCD and the CCD output was analyzed to determine the relative intensity within the mode field. This result is shown in Figure 5.4 and appears to be consistent with the mode profiles obtained at NRC and resembles the field profiles predicted in the simulations. 


\section{Mode Profile}

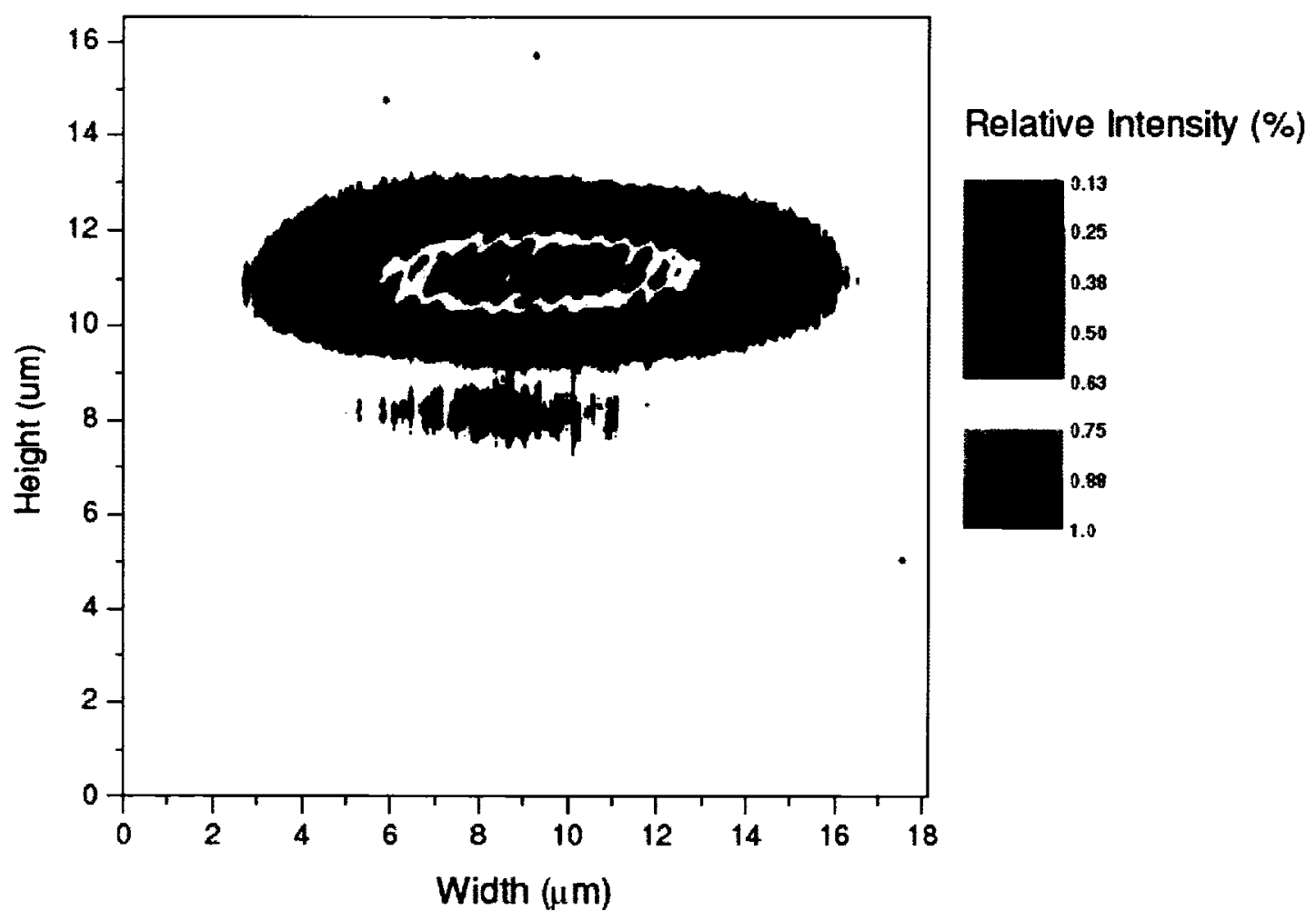

Figure 5.4: Intensity distribution within guided mode in $3 \mu \mathrm{m}$ wide LOCOS isolated rib waveguide tapered to a final width of $15 \mu \mathrm{m}$.

In this case, we can see that we must have the single mode operation predicted by the simulations in section 3.1.

\subsection{Propagation Losses in $3 \mu \mathrm{m}$ and $4 \mu \mathrm{m}$ LOCOS waveguides (McMaster)}

The cutback method was used to estimate the propagation losses in the LOCOS waveguides for the 3 and $4 \mu \mathrm{m}$ wide ribs. Under this method, light is coupled into these waveguides (\#46 - 54 and \#28-36 on mask) and repeated power measurements are made on each waveguide to obtain a statistical average value of transmitted power. The sample was subsequently cleaved again multiple times to a shorter length and the process of coupling light into the waveguides and averaging many power measurements was 
Chapter 5 Results and Discussion

repeated at each sample length. Finally, to determine the estimated propagation loss these power measurements were plotted on a decibel scale versus the length of the sample from which they were obtained and a line of best fit to these data points was drawn. The loss was then calculated as the slope of this line.

The power measurements and the line of best fit for the $3 \mu \mathrm{m}$ and $4 \mu \mathrm{m}$ waveguide ribs are shown in Figure 5.5 and Figure 5.6. The slopes and intercepts as well as the standard error of these slopes and intercepts for the lines of best fit were determined using linear regression analysis [Bev92]. The line of best fit for the power loss in the $3 \mu \mathrm{m}$ wide LOCOS isolated waveguides was found to have a slope of $-0.9 \pm 0.2 \mathrm{~dB} / \mathrm{cm}$ and an intercept of $-13.9 \pm 0.4 \mathrm{dBm}$. Similarly, the line of best fit for the power loss in the $4 \mu \mathrm{m}$ wide LOCOS isolated waveguides was found to have a slope of $0.35 \pm 0.09 \mathrm{~dB} / \mathrm{cm}$ and an intercept of $-16.7 \pm 0.2 \mathrm{dBm}$. 
Chapter 5 Results and Discussion

Estimated Propagation Loss in 3 um Waveguides

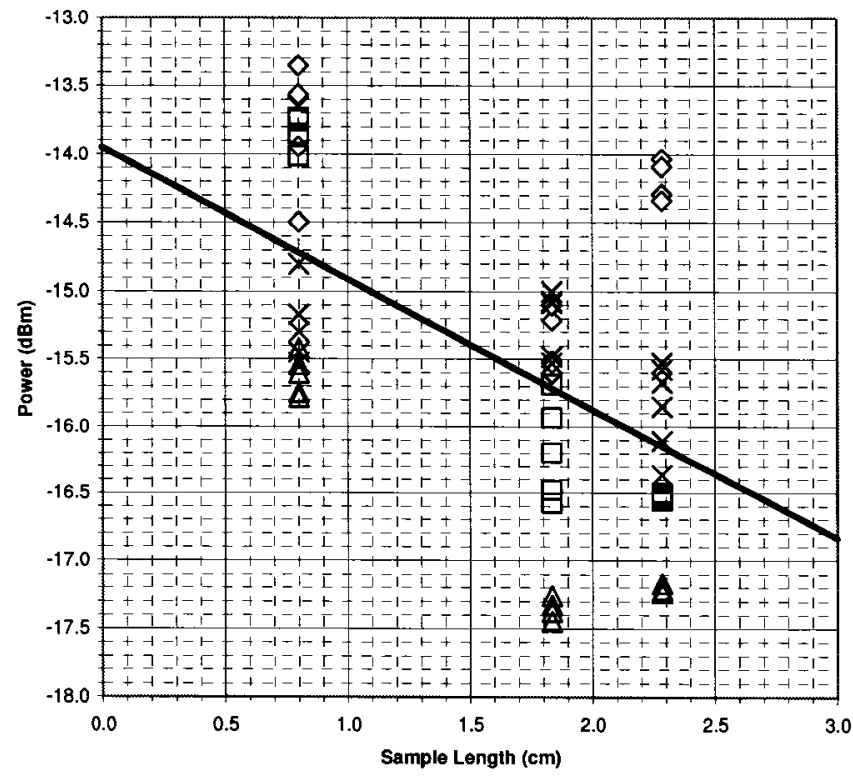

- Measured Power in Waveguide $46(\mathrm{dBm})$

c Measured Power in Waveguide $47(\mathrm{dBm})$

$X$ Measured Power in Waveguide $49(\mathrm{dBm})$

$\Delta$ Measured Power in Waveguide $50(\mathrm{dBm})$

Best Fit with Floating Intercept

Figure 5.5: Measured power $(\mathrm{dBm})$ vs. sample length $(\mathrm{cm})$ from cutback measurements on $3 \mu \mathrm{m}$ wide $L O C O S$ waveguides

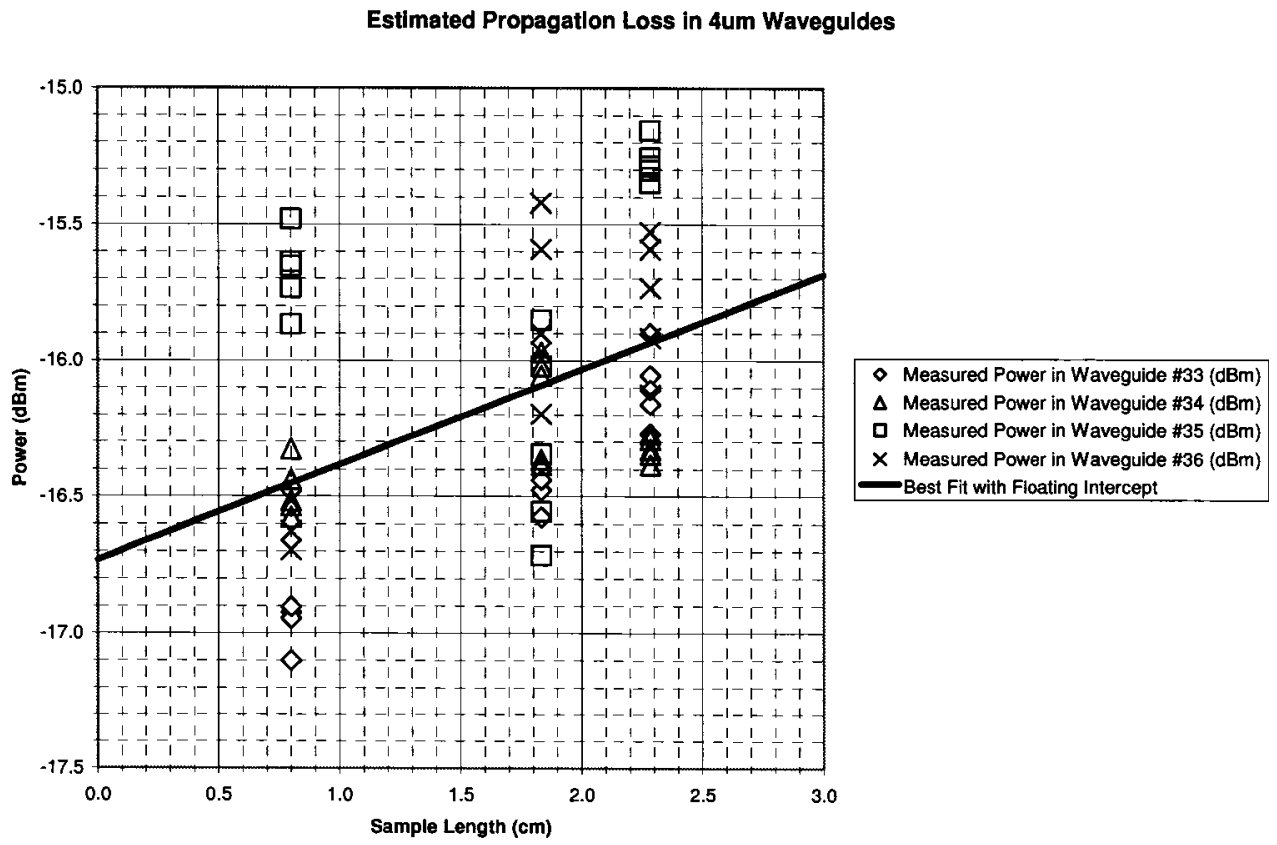

Figure 5.6 : Measured power $(\mathrm{dBm})$ vs. sample length $(\mathrm{cm})$ from cutback measurements on $4 \mu \mathrm{m}$ wide LOCOS waveguides 
Chapter 5 Results and Discussion

Based on these analyses, we notice that the slope obtained for the $4 \mu \mathrm{m}$ wide waveguides actually seems to indicate a power gain rather than a power loss as would be expected in the waveguide. This is clearly a physical impossibility and this result demonstrates the difficulty in making measurements of relatively small losses using the cutback technique. In spite of the large uncertainty in the measurements, it appears that we can confidently state that the loss in LOCOS isolated waveguides is at worst in the range of $1 \mathrm{~dB} / \mathrm{cm}$.

\subsection{Unbalanced Mach-Zehnder Interferometer Performance (NRC)}

To verify that the LOCOS isolated waveguide structures are suitable for use in optical devices and, in particular, in optical devices with splitter structures, the performance of the unbalanced Mach-Zehnder Interferometer device was verified.

The wavelength of light was swept from $1470 \mathrm{~nm}$ to $1580 \mathrm{~nm}$ in increments of $0.2 \mathrm{~nm}$ and the optical power at the output of these unbalanced Mach-Zehnder interferometers was measured for each wavelength with both the TE and TM polarization. The results are shown in the graph of Figure 5.7. 


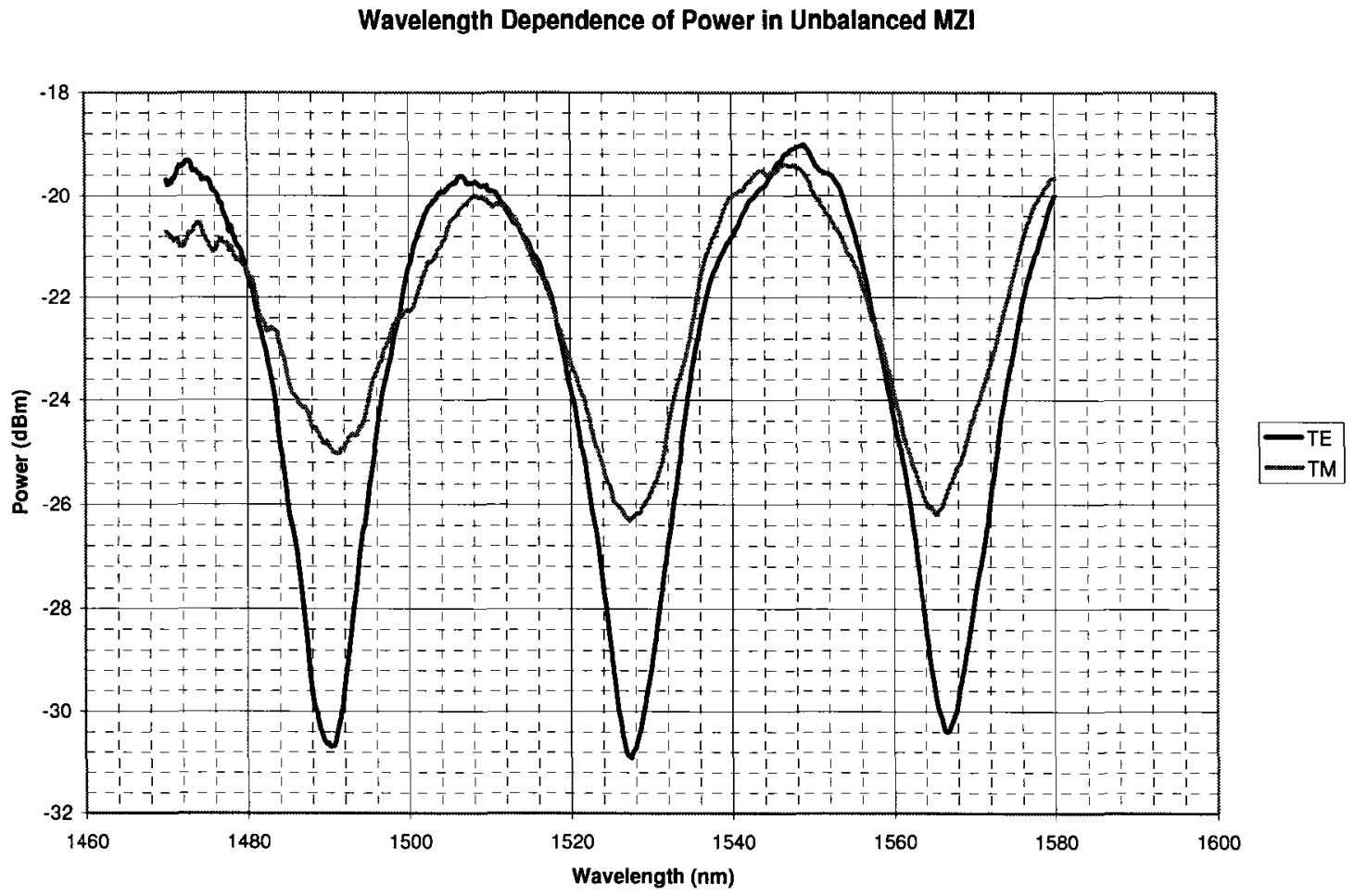

Figure 5.7: Power vs. wavelength for light of both polarizations launched into unbalanced MZI device

Based on the above graph (Figure 5.7), it appears that the modulation period of these unbalanced MZI devices is approximately $40 \mathrm{~nm}$. In this device, the modulation depth for the TE polarization is approximately $11 \mathrm{~dB}$ while the modulation depth for the TM polarization is approximately $6 \mathrm{~dB}$. These modulation depths do seem to agree reasonably well in terms of magnitude with those obtained in the simulation (see section 3.3) where modulation depths on the order of $12 \mathrm{~dB}$ were predicted. However, in this case, practical considerations such as the power distribution between the two branches of the MZI due to manufacturing concerns such as loading effects in the etching process and sidewall roughness can impact the modulation depths obtained and explain the observed difference in extinction for the two polarizations. 
Chapter 5 Results and Discussion

\subsection{Balanced Y-splitter Performance (NRC)}

One final test of the quality of the splitters that can be made with the LOCOS isolation technique involved launching $1550 \mathrm{~nm}$ light of both the TE and TM polarizations into the $3 \mu \mathrm{m}$ Y-splitter device (\#55 on the mask) and measuring the output power at each of the two output ports. The results obtained are summarized in Table 5.1.

\begin{tabular}{|l|l|l|}
\hline Branch & Polarization & Measured Output Power (dBm) \\
\hline 1 & TE & -24.45 \\
\hline 2 & TE & -30.40 \\
\hline 1 & TM & -26.12 \\
\hline 2 & TM & -25.93 \\
\hline
\end{tabular}

Table 5.1: Output power level obtained with $1550 \mathrm{~nm}$ light of both polarizations launched into $3 \mu \mathrm{m}$ Y-splitter

Based on these results, we see that while there appears to be an approximately equal splitting of the light in the TM polarization, the TE polarization suffers from a very unequal splitting of the input power. The majority of the output power appears in the first branch while $6 \mathrm{dBm}$ less power appears in the second branch of the Y-splitter at 1550 $\mathrm{nm}$. This is likely due to a problem with the split formation or a change in the properties of the rib in one branch as compared to the other. Since we have seen that the effective index of a mode differs according to rib width, it may be that the optical mode launched in one arm of the device is not as well confined in the TE polarization as with the TM due to a narrowing of the rib, causing the polarization dependent loss observed. 


\section{Chapter 6 Conclusion and Recommendations}

\subsection{Thesis Summary}

This thesis set out to show that the local oxidation of silicon (LOCOS) process used for isolating active devices in older CMOS technologies can be employed to define the ridge of an optical waveguide in silicon on insulator (SOI) while maintaining a planar silicon surface suitable for integrating electronic components. The main advantage to this approach is the ability to integrate optical components along with high-quality electronics on a single chip.

Three LOCOS processes were considered for this project. The first of these processes is the standard LOCOS process in which a combination of a thin layer of silicon dioxide and a thick layer of silicon nitride are used to control the oxidation of the silicon beneath them. Steeper waveguide sidewalls could be achieved through the use of modified LOCOS processes such as polysilicon buffered LOCOS (PBL) or sealed-interface local oxidation (SILO) that are known to have a substantially smaller encroachment than the standard LOCOS process.

Numerical solution of the Helmholtz equation was undertaken to find optical waveguide geometries that can be produced with LOCOS methods which show desirable single-mode propagation in the $1550 \mathrm{~nm}$ optical telecommunications band. Additional optical modeling was also done to investigate the birefringence in the waveguide structures defined with each of the LOCOS processes. 
Chapter 6 Conclusion and Recommendations

In addition, process flow modeling was done to determine the extent of the encroachment and the angle of the sidewall produced as well as determining the magnitude of the mechanical stresses which can contribute to the formation of dislocations or to waveguide birefringence that can affect the performance of the fabricated optical and electrical devices. In this study, the stresses in the oxide layer for all LOCOS processes were found to be reasonably small in magnitude when compared to those reported in the literature to produce significant birefringence [Ye04].

Waveguides with drawn ridge widths ranging from $3 \mu \mathrm{m}$ to $5 \mu \mathrm{m}$ were fabricated on bond-and-etchback (BESOI) and smart-cut SOI substrates with silicon film thicknesses of $2.5 \mu \mathrm{m}$ and $3.4 \mu \mathrm{m}$ respectively. Using a $1 \mu \mathrm{m}$ field oxidation, waveguides with drawn widths from $3 \mu \mathrm{m}$ to $5 \mu \mathrm{m}$ resulted in final ridge widths of approximately $2 \mu \mathrm{m}$ to $4 \mu \mathrm{m}$ and a ridge height of approximately $0.5 \mu \mathrm{m}$. The propagation losses in these waveguides were found to be less than $1 \mathrm{~dB} / \mathrm{cm}$.

In addition, unbalanced Mach-Zehnder interferometers (MZI) and Y-splitters were also fabricated on the same substrates with this process to demonstrate the ability to produce complex waveguide devices with this LOCOS approach. The Y-splitters showed an approximately even distribution of the input optical power between the two output branches at $1550 \mathrm{~nm}$ and the unbalanced MZI showed an extinction of 6-10 dB with a modulation period of approximately $40 \mathrm{~nm}$ over the range from 1470 to $1580 \mathrm{~nm}$ for both the TE and TM polarizations.

In summation, the LOCOS isolated optical rib waveguide structure has been shown to be a practical approach to achieving the surface planarity required to integrate optical and electronic components on the same chip in older CMOS technologies and high-voltage 
Chapter 6 Conclusion and Recommendations

CMOS technologies where coarser device geometries exist. The smooth sidewall surfaces obtained as a result of the oxidation are responsible for lower scattering losses, while the stresses due to oxidation are still low enough to avoid effects such as excessive birefringence and dislocation damage that can be harmful to optical and electronic device performance. A paper based on this work has been accepted for presentation at the SPIE Photonics West Conference in January, 2007.

\subsection{Recommendations for Future Work}

One of the major advantages of the LOCOS defined waveguide discussed in this thesis is its ease of use for the monolithic integration of optical and electrical components. In particular, the use of the LOCOS process for electrical device isolation as well as optical rib formation results in a savings on masks and photolithography steps which can reduce fabrication process complexity and cost. Therefore, it seems logical that remaining research efforts should concentrate on demonstrating this ability to integrate electrical and optical components on a single die with this process.

To accomplish this task, additional process modeling work should be done with a focus on determining suitable electrical characteristics for transistors in the CMOS process in SOI substrates.

Another area for investigation would involve optical modeling and measurement to study the impact of stresses in the oxide isolation regions on the waveguide birefringence and their effect on complex waveguide structures. 


\section{Appendix A: Simulation Parameter Details}

\begin{tabular}{|l|l|}
\hline & Grid Points \\
\hline Horizontal Mesh & $200(350)$ \\
\hline Vertical Mesh - Silicon Core & $20(28)$ \\
\hline Vertical Mesh - Buried Oxide & $4(4)$ \\
\hline Vertical Mesh - Substrate & $21(21)$ \\
\hline Vertical Mesh - Pad oxide & $2(2)$ \\
\hline Vertical Mesh - Air & $21(21)$ \\
\hline
\end{tabular}

Table A.1: Simulation grid for Apollo simulations of LOCOS waveguide in SEH (SOITEC) substrates 


\section{Bibliography}

[Agra02] G.P. Agrawal, Fiber-Optic Communications Systems 3rd Edition, New York: Wiley, 2002.

[Ant79] D.A. Antoniadis and R.W. Dutton, "Models for Computer Simulation of Complete IC Fabrication", IEEE Trans. Electron Devices, vol. ED-26, no. 4, pp. 490-500, Apr. 1979.

[Apo02] Apollo Inc., Apollo Photonic Solutions Suite (APSS) User Manual, 2002.

[Bev92] P.R. Bevington and D.K. Robinson, Data Reduction and Error Analysis for the Physical Sciences $2^{\text {nd }}$ Edition, New York:

McGraw-Hill, 1992.

[Bri05] M.C. Brito, J.P. Pereira, J. Maia Alves, J.M. Serra, and A. M.

Vallera, "Measurement of residual stress in multicrystalline silicon ribbons by a self-calibrating infrared photoelastic method", Rev.

Sci. Instrum., vol. 76, no. 1, pp. , Jan. 2005.

[Car05] Y. Carts-Powell, "Silicon photonics moves toward practical use", Laser Focus World, November 2005. [Online]. Available: http://lfw.pennnet.com/articles/article display.cfm?Section=ARC $\underline{\text { HI\&C}=\text { News\&ARTICLE_ID }=241174 \& \text { KEYWORDS }=\text { silicon\%20 }}$ photonics $\& \mathrm{p}=12 \& \mathrm{pc}=\mathrm{ENL}$

[Chi86] K.S. Chiang, "Analysis of optical fibers by the effective-index method", Appl. Opt., vol. 25, no. 3, pp. 348-354, Feb. 1986.

[Chi96] K.S. Chiang, "Analysis of the Effective-Index Method for the Vector Modes of Rectangular-Core Dielectric Waveguides", IEEE Trans. Microwave Theory Tech., vol. 44, no. 5, pp. 692-700, May 1996.

[Chu91] Y. Chung and N. Dagli, “Analysis of Z-invariant and Z-variant Semiconductor Rib Waveguides by Explicit Finite Difference Beam Propagation Method with Nonuniform Mesh Configuration", IEEE J. Quantum Electron., vol. 27, no. 10, pp. 2296-2305, Oct. 1991.

[Cisco04] Cisco Systems, Introducing DWDM, 7 June 2004. [Online]. Available: http://www.cisco.com/univercd/cc/td/doc/product $/ \mathrm{mels} / \mathrm{cm} 1500 / \mathrm{d}$ wdm/dwdm fns.htm 
[Com04] Comsol AB, FEMLAB User's Guide, 2004.

[Dal04] Dalsa Semiconductor, CO8E $0.8 \mu \mathrm{m} 20 \mathrm{~V}$ BCD Process, 5 October 2004. [Online]. Available:

http://www.dalsasemi.com/PDFs/00145-01 03-70 C08E flyer.pdf

[Eer77] E.P. EerNisse, "Viscous flow of thermal $\mathrm{SiO}_{2}$ ", Appl. Phys. Lett., vol. 30, no. 6, pp. 290-293, Mar. 15, 1977.

[Gei03] M. Geis, S. Spector, R. Williamson, T. Lyszczarz, S.G. Johnson, and M.L. Povinelli, "Photonic Components for RF Filtering and Wide-Band Antenna Nulling", Optics in Computing, vol. 90, pp. 69-71, 2003.

[Hat78] T. Hattori, " $\mathrm{HCl}$ oxidation conditions for stacking-fault nuclei gettering and for silicon etching", J. Appl. Phys., vol. 49, no. 5, pp. 2994-2995, May 1978.

[Hau05] T. Hausken, "Silicon nanophotonics: A Mazerati or a Prius?", Laser Focus World, November 2005. [Online]. Available: http://lfw.pennnet.com/articles/article display.cfm?Section=ARC HI\&C=Dept\&ARTICLE ID=241173\&KEYWORDS=silicon\%20 photonics\&p=12\&pc=ENL

[Ho83] C. Ho, J.D. Plummer, S.E. Hansen, and R.W. Dutton, "VLSI Process Modeling - SUPREM III", IEEE Trans. Electron Devices, vol. ED-30, no. 11, pp. 1438-1453, Nov. 1983.

[ITRS05] International Technology Roadmap for Semiconductors. Semiconductor Industry Association, 2005. [Online]. Available: http://www.itrs.net/Links/2005ITRS/Home2005.htm.

[Ja198] B. Jalali, S. Yegnanarayanan, T. Yoon, T. Yoshimoto, I. Rendina, and F. Coppinger, "Advances in Silicon-on-Insulator Optoelectronics," J. Sel. Topics Quantum Electron., vol. 4, no. 6, pp. 938-947, Nov./Dec. 1998.

[Kos85] M. Koshiba, K. Hyata and M. Suzuki, "Finite-Element Formulation in Terms of the Electric-Field Vector for Electromagnetic Waveguide Problems", IEEE Trans. Microwave Theory Tech., vol. MTT-33, no. 10, pp.900-905, Oct. 1985.

[Kos92] M. Koshiba, H. Saitoh, M. Eguchi and K. Hirayama, "Simple scalar finite element approach to optical rib waveguides", Proc. IEEE, vol. 139, pt. J, no. 2, pp. 166-171, Apr. 1992. 
[Kya05] I. Kyat, A. Aydinli, and N. Dagli, "A Compact Silicon-onInsulator Polarization Splitter," IEEE Photon. Technol. Lett., vol. 17, no. 1, pp. 100-102, Jan. 2005.

[Lai99] L. Lai and E.A. Irene, "Limiting Si/SiO2 surface roughness resulting from thermal oxidation", J. Appl. Phys., vol. 86, no. 3, pp. 1729-1735, Aug.1999.

[Lee01] K.K. Lee, D.R. Lim, and L.C. Kimerling, "Fabrication of ultralowloss $\mathrm{Si} / \mathrm{SiO} 2$ waveguides by roughness reduction," Opt. Lett., vol. 26, no. 23, pp. 1888-1890, 2001.

[Lia92] H. Liang, Y. Pan, S. Zhao, G. Qin, and K. K. Chin, "Twodimensional state of stress in a silicon wafer", J. Appl. Phys., vol. 71, no. 6, pp. 2863-2870, March 1992.

[Mab81] N. Mabaya, P.E. Lagasse and P. Vandenbulcke, "Finite Element Analysis of Optical Waveguides", IEEE Trans. Microwave Theory Tech., vol. MTT-29, no. 6, pp. 600-605, June 1981.

[Oka00] K. Okamoto, Fundamentals of Optical Waveguides, San Diego: Academic Press, 2000.

[Pear00] M.R.T. Pearson, A. Bezinger, A. Delage, J.W. Fraser, S. Janz, P.E. Jessop, D.-X. Xu, "Arrayed waveguide demultiplexers in siliconon-insulator”, Proc. SPIE, v. 3958, pp. 11-18, 2000.

[Pear01] M.R.T. Pearson, P.E. Jessop, D.M. Bruce, S. Wallace, P. Mascher, and J. Ojha, "Fabrication of SiGe Optical Waveguides Using VLSI Processing Techniques", J. Lightw. Technol., vol. 19, no. 3, pp. 363-370, Mar. 2001.

[Plu00] J.D. Plummer, Silicon VLSI Technology: Fundamentals, Practice and Modeling, Upper Saddle River NJ: Prentice Hall, 2000.

[Pog98] S.P. Pogossian, L. Vescan, and A. Vonsovici, "The Single-Mode Condition for Semiconductor Rib Waveguides with Large Cross Section," J. Lightw. Technol., vol. 16, no. 10, pp. 1851-1853, Oct. 1998.

[Reed04] G.T. Reed and A.P. Knights, Silicon Photonics, West Sussex, England: John Wiley and Sons, 2004.

[Sa104] M. Salib, L. Liao, R. Jones, M. Morse, A. Liu, D. Samara-Rubio, D. Alduino, M. Paniccia, "Silicon Photonics", Intel Technology Journal, vol.8 no.2, May 10, 2004. pp.143-160 
[Shi80] K. Shibata and K. Taniguchi, "Generation Mechanism of Dislocations in Local Oxidation of Silicon", J. Electrochem. Soc., vol. 127, no. 6, pp. 1383-1387, Jun. 1980.

[Soi05] Unibond overview. SOITEC, Bernin, France. 2005. [Online]. Available: http://www.soitec.com/en/products/p_1.htm

[Sor91] R.A. Soref, J. Schmidtchen, and K. Petermann, "Large SingleMode Rib Waveguides in GeSi-Si and $\mathrm{Si}^{-o n S i O}{ }_{2}$," IEEE J. Quantum Electron., vol. 27, no. 8, pp. 1971-1974, Aug. 1991.

[Ste88] M.S. Stern, "Semivectorial polarised finite difference method for optical waveguides with arbitrary index profiles", Proc. IEEE, vol. 135, Pt. J, no. 1, pp. 56-63, Feb. 1988.

[Syn05] Synopsys Inc., Taurus TSUPREM-4 User Guide, October 2005.

[Tur04] A. Turnbull, Trench Isolated Silicon-on-Insulator Optical Rib Waveguides, Ottawa: Carleton University, 2004.

[Van81] J. Van Roey, J. van der Donk, and P.E. Lagasse, "Beampropagation method: analysis and assessment", J. Opt. Soc. Am., vol. 71, no. 7, pp. 803-810, July 1981.

[Wolf90] S. Wolf, Silicon Processing for the VLSI era Volume 2:Process Integration, Sunset Beach CA: Lattice Press, 1990.

[Wolf95] S. Wolf, Silicon Processing for the VLSI era Volume 3: The Submicron MOSFET, Sunset Beach CA: Lattice Press, 1995.

[Ye04] D.-X. Xu, P. Cheben, D. Dalacu, A. Delâge, S. Janz, B. Lamontagne, M.-J. Picard, and W. N. Ye, "Eliminating the birefringence in silicon-on-insulator ridge waveguides using the cladding stress," Opt. Lett. , vol. 29, pp. 2384-2386, 2004.

[Ye05] W. N. Ye, D.-X. Xu, S. Janz, P. Cheben, M.-J. Picard, B. Lamontagne, N. G. Tarr, "Birefringence Control Using Stress Engineering for Silicon-on-insulator (SOI) Waveguides," $J$. Lightw. Technol., vol. 23, pp. 1308-1318, 2005. 